$$
\begin{aligned}
& \text { CU TP } 6 \\
& \text { SW } 3413 \\
& C U-T P-622 \\
& R U-94-1-B
\end{aligned}
$$

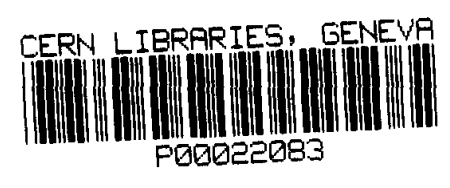

\title{
BOSONIZATION OF LATTICE FERMIONS
}

\author{
R. FRIEDBERG, T. D. LEE \\ COLUMBIA UNIVERSITY, NEW YORK, N.Y. 10027 \\ AND \\ H. C. REN
}

THE ROCKEFELLER UNIVERSITY, NEW YORK, N.Y. 10021

THIS RESEARCH WAS SUPPORTED IN PART BY THE U.S. DEPARTMENT OF ENERGY UNDER GRANTS DE-FG02-92 ER40699 AND DOE-91ER40651, TASK B. 


\begin{abstract}
For any purely fermionic system on a lattice in arbitrary space-dimension we prove that there is a different but well-defined system on the same lattice, consisting of both bona fide fermions and bosons with an interaction depending on a parameter $\lambda$, which characterizes a same-site repulsion between particles. The energy spectrum and the scattering matrix of the former are identical to those in the finite energy sector of the latter, in the limit $\lambda \rightarrow \infty$ (i.e., hard core repulsion). This general theorem is applied to a resonance-boson model in a lattice fermion system, whose solution may have relevance to high $T_{c}$ superconductivity.
\end{abstract}




\subsection{Lemma}

The basis of the equivalence theorem is the following lemma, proved recently ${ }^{1}$ for the equivalence between spin waves and lattice bosons. Take a general $(d+$ $\left.d^{\prime}\right) \times\left(d+d^{\prime}\right)$ hermitian matrix

$$
\mathcal{H}=\lambda \mathcal{H}_{0}+\mathcal{H}_{1}
$$

where $\mathcal{H}_{0}$ and $\mathcal{H}_{1}$ are both independent of the parameter $\lambda$ and $\mathcal{H}_{0}$ is of the form

$$
\mathcal{H}_{0}=\left(\begin{array}{ll}
0 & 0 \\
0 & X
\end{array}\right)
$$

in which 0 in the upper left corner denotes a $d \times d$ null matrix and $X$ is a $d^{\prime} \times d^{\prime}$ positive definite (and otherwise arbitrary) hermitian matrix. $\mathcal{H}_{1}$ can be any $(d+$ $\left.d^{\prime}\right) \times\left(d+d^{\prime}\right)$ hermitian matrix, which can always be written as

$$
\mathcal{H}_{1}=\left(\begin{array}{cc}
W & Y^{\dagger} \\
Y & Z
\end{array}\right)
$$

with $W$ and $Z$ being $d \times d$ and $d^{\prime} \times d^{\prime}$ hermitian matrices.

Write the normalized eigenvector of $\mathcal{H}$ in the form

$$
\psi=\left(\begin{array}{l}
\chi \\
\phi
\end{array}\right)
$$

with $\chi$ and $\phi$ having $d$ and $d^{\prime}$ components. We can express the eigenvalue equation $\mathcal{H} \psi=\omega \psi$ in terms of $\chi$, by eliminating $\phi$ :

$$
(W-\omega) \chi=\mathcal{H}^{\prime} \chi
$$

where $\mathcal{H}^{\prime}=Y^{\dagger} X^{-1 / 2}(\lambda+M)^{-1} X^{-1 / 2} Y$ and $M=X^{-1 / 2}(Z-\omega) X^{-1 / 2}$, both $d \times d$ hermitians. Separate all the eigenvalues $\omega$ into two sets $\left\{\Lambda_{a}\right\}$ and $\left\{\mathcal{E}_{i}\right\}$ where, as $\lambda \rightarrow \infty, \Lambda_{a}$ is $O(\lambda)$ or larger and $\varepsilon_{i}$ is $o(\lambda)$, with $a=1, \cdot, N$, $i=1, \cdot \cdot n$ and $N+n=d+d^{\prime}$. For $\omega=\mathcal{E}_{i}$, we have, in the limit $\lambda \rightarrow \infty$. $\lambda+M \rightarrow \lambda$ and $\mathcal{H}^{\prime}=O\left(\lambda^{-1}\right) \rightarrow 0$; therefore $\mathcal{E}_{i}$ must become one of the eigenvalues $E_{\nu}$ of $W$.

$$
W \chi_{\nu}=E_{\nu} \chi_{\nu}
$$




\section{THE EQUIVALENCE THEOREM}

Take any fermion system on a lattice (regular or random, in any dimension) of sites $j=1,2, \cdots, \mathcal{N}$. The annihilation and creation operators on each site $j$ are $\alpha_{j \sigma}$ and $\alpha_{j \sigma}^{\dagger}$, satisfying the usual anticommutation relations

$$
\left\{\alpha_{j \sigma}, \alpha_{j^{\prime} \sigma^{\prime}}^{\dagger}\right\}=\delta_{j j^{\prime}} \delta_{\sigma \sigma^{\prime}}
$$

and

$$
\left\{\alpha_{j \sigma}, \alpha_{j^{\prime} \sigma^{\prime}}\right\}=0
$$

where ${ }^{\dagger}$ denotes the hermitian conjugate, $\sigma$ and $\sigma^{\prime}$ are the spin indices and $\delta_{j j^{\prime}}, \delta_{\sigma \sigma^{\prime}}$ the Kronecker symbols. The Hamiltonian

$$
h=h\left(\alpha_{j \sigma}, \alpha_{j \sigma}^{\dagger}\right)
$$

can be an arbitrary hermitian function of $\alpha_{j \sigma}$ and $\alpha_{j \sigma}^{\dagger}$. As we shall see, for every such $h$, there is another system in which both fermionic operators $a_{j \sigma}$ and $a_{j \sigma}^{\dagger}$ and bosonic operators $b_{j}$ and $b_{j}^{\dagger}$ exist on each lattice site $j$, with

$$
\begin{gathered}
\left\{a_{j \sigma}, a_{j^{\prime} \sigma^{\prime}}^{\dagger}\right\}=\delta_{j j^{\prime}} \delta_{\sigma \sigma^{\prime}} \\
\left\{a_{j \sigma}, a_{j^{\prime} \sigma^{\prime}}\right\}=0 \\
{\left[b_{j}, b_{j^{\prime}}^{\dagger}\right]=\delta_{j j^{\prime}}}
\end{gathered}
$$

and $b_{j}$ commutes with $b_{j^{\prime}}, a_{j^{\prime} \sigma}$ and $a_{j^{\prime} \sigma}^{\dagger}$. The Hamiltonian

$$
H=H\left(a_{j \sigma}, b_{j}, a_{j \sigma}^{\dagger}, b_{j}^{\dagger}, \lambda\right)
$$

of this new system is a well-defined hermitian function, depending on $a_{j \sigma}, b_{j}$, their hermitian conjugates and a parameter $\lambda$. In the limit $\lambda \rightarrow \infty$, the energy spectrum and the $S$-matrix of the finite sector of $H$ are identical to those of $h$. In what follows, we shall first establish the equivalence theorem in this section. For illustration we give a resonance boson model; its details will be analyzed in Sections 2-4. 
$\chi \rightarrow$ the corresponding eigenvector $\chi_{\nu}$ and, as can readily be verified, $\phi \rightarrow 0$. Since altogether $W$ has $d$ eigenvalue solutions, $n \leq d$. As $\lambda \rightarrow \infty$, from (1.5)(1.6) we see that $\operatorname{det} \mathcal{H}=O\left(\lambda^{d^{\prime}}\right)$; on the other hand, $\prod_{a=1}^{N} \Lambda_{a}$ is $O\left(\lambda^{N}\right)$ or larger. Therefore, $N \leq d^{\prime}$. Thus, $N=d^{\prime}, n=d$ and we obtain the following lemma:

In the limit $\lambda \rightarrow \infty$, all the eigenvalues in the finite sector of $\mathcal{H}$ are the same $E_{\nu}$ of $W$, with the corresponding eigenvectors

$$
\left(\begin{array}{c}
\chi_{\nu} \\
0
\end{array}\right)
$$

determined by the same $\chi_{\nu}$ of (1.7). Furthermore, each $\Lambda_{a}$ is $O(\lambda)$. (If det $W=0$, the argument should be modified by considering $\operatorname{det}(\mathcal{H}+C)$ where $C$ is a constant, independent of $\lambda$, such that $\operatorname{det}(W+C) \neq 0$.)

\subsection{Equivalence}

For a finite lattice of $\mathcal{N}$ sites, the pure fermion system (defined by the Hamiltonian $h,(1.2)$ ) is spanned by a Hilbert space $\Omega_{h}$ of dimension $4^{\mathcal{N}}$, since on each site $i$, there can be zero, one (spin $\uparrow$ or $\downarrow$ ) and two (spin singlet) particles. Decompose $\Omega_{h}$ into

$$
\Omega_{h}=\Omega_{F}+\Omega_{F}^{\prime}
$$

with dimensions

$$
\operatorname{dim} \Omega_{F}=3^{\mathcal{N}}
$$

and

$$
\operatorname{dim} \Omega_{F}^{\prime}=4^{\mathcal{N}}-3^{\mathcal{N}}
$$

the subspace $\Omega_{F}$ is defined by restricting the number of fermions on each site to only 0 or 1 , and $\Omega_{F}^{\prime}$ is the remainder. Thus, a state vector in $\Omega_{F}$ can always be written in the form

$$
\left.\mid F)=\prod_{i=1}^{\mathcal{N}}\left(c_{0}(i)+c_{\uparrow}(i) \alpha_{i \uparrow}^{\dagger}+c_{\downarrow}(i) \alpha_{i \downarrow}^{\dagger}\right) \mid 0\right)
$$

where $c_{0}(i)$ and $c_{\sigma}(i)$ are $c$ numbers and $\left.\mid 0\right)$ is the vacuum state of $h$, defined by 


$$
\alpha_{i \sigma}(0)=0 \quad \text { for all } i \text { and } \sigma \text {. }
$$

Any state vector in $\Omega_{F}^{\prime}$ must consist of one or more sites, say

$$
j_{1}, j_{2}, \cdots, j_{m} \equiv\{j\}
$$

that are occupied by two fermions. Each such state is of the form

$$
\left.\left|F^{\prime}\right\rangle=\alpha_{j_{1} \uparrow}^{\dagger} \alpha_{j_{1} \downarrow}^{\dagger} \alpha_{j_{2} \uparrow}^{\dagger} \alpha_{j_{2} \downarrow}^{\dagger} \cdots \alpha_{j_{m} \uparrow}^{\dagger} \alpha_{j_{m} \downarrow}^{\dagger} \prod_{i \not \subset\{j\}}\left(c_{0}(i)+c_{\uparrow}(i) \alpha_{i \uparrow}^{\dagger}+c_{\downarrow}(i) \alpha_{i \downarrow}^{\dagger}\right) \mid 0\right) .
$$

The Hilbert space $\Omega_{H}$ of the boson-fermion Hamiltonian (1.4) is much larger, since on each lattice site there can be, in addition to the fermionic degrees of freedom, any number of bosons. We can decompose

$$
\Omega_{H}=\Omega_{f}+\Omega_{f b}+\Omega_{f b}^{\prime} .
$$

There is a one-to-one correspondence between the vectors in $\Omega_{F}$ and $\Omega_{f}$, and likewise between those in $\Omega_{F}^{\prime}$ and $\Omega_{f b}$. A state vector $\left.\mid F\right)$ in $\Omega_{F}$ corresponds to

$$
\left|f>=\prod_{i=1}^{\mathcal{N}}\left(c_{0}(i)+c_{\uparrow}(i) a_{i \uparrow}^{\dagger}+c_{\downarrow}(i) a_{i \downarrow}^{\dagger}\right)\right| 0>
$$

in $\Omega_{f}$ where $c_{0}(i), c_{\uparrow}(i)$ and $c_{\downarrow}(i)$ are the same $c$ number amplitudes in (1.11) and the vacuum state $\mid 0>$ is defined by

$$
a_{i \sigma}\left|0>=b_{i}\right| 0>=0 \quad \text { for all } i \text { and } \sigma .
$$

Similarly, a state vector (1.14) in $\Omega_{F}^{\prime}$ corresponds to

$$
\left|f b>=b_{j_{1}}^{\dagger} b_{j_{2}}^{\dagger} \cdots b_{j_{m}}^{\dagger} \prod_{i \not \subset\{j\}}\left(c_{0}(i)+c_{\uparrow}(i) a_{i \uparrow}^{\dagger}+c_{\downarrow}(i) a_{i \downarrow}^{\dagger}\right)\right| 0>
$$

in $\Omega_{f b}$. Thus, $\Omega_{f}+\Omega_{f b}$ denotes all states in which the number of particles on each site can only be zero or one, and the latter can be either a fermion ( $\uparrow$ or $\downarrow$ ) or a boson. The dimension of $\Omega_{f}+\Omega_{f b}$ is $4^{\mathcal{N}}$, the same as $\Omega_{h}$, and that of $\Omega_{f}$ 
is $3^{\mathcal{N}}$, the same as $\Omega_{F}$. In (1.15), any state vector in the (remaining) subspace $\Omega_{f b}^{\prime}$ denotes a configuration in which there are two or more particles on at least one site: for the former, two particles on one site, the two can be a fermion spin singlet pair, or one boson and one fermion, or two bosons; for the latter, there may be more bosons on the same site.

By identifying $4^{\mathcal{N}}=d$ of the lemma, with $\Omega_{f}+\Omega_{f b}$ as the subspace, we denote $\mathcal{H}$ of (1.5) by $H$ of (1.4); we write

$$
H=H_{\text {core }}+\mathcal{H}_{1}
$$

where

$$
H_{\text {core }} \equiv \lambda \sum_{j}\left(\hat{\xi}_{0} a_{j \uparrow}^{\dagger} a_{j \downarrow}^{\dagger} a_{j \downarrow} a_{j \uparrow}+\hat{\eta}_{0} a_{j \sigma}^{\dagger} b_{j}^{\dagger} b_{j} a_{j \sigma}+\frac{1}{4} \hat{\zeta}_{0} b_{j}^{\dagger 2} b_{j}^{2}\right)=\lambda \mathcal{H}_{0}
$$

with $\hat{\xi}_{0}, \hat{\eta}_{0}$ and $\hat{\zeta}_{0}$ always kept finite $>0$, so that the limit $\lambda \rightarrow \infty$ implies each term inside the parenthesis $\rightarrow \infty$. Every state vector $|c\rangle$ in $\Omega_{f}+\Omega_{f b}$ corresponds uniquely to a state vector $\mid c$ ) in $\Omega_{h}$, and vice versa, through the correspondence either between (1.11) and (1.16), or that between (1.14) and (1.18). For any two basis vectors $\mid c)$ and $\left.\mid c^{\prime}\right)$ in the finite Hilbert space $\Omega_{h}$, we can always construct an $\mathcal{H}_{1}$ so that its matrix element between the corresponding basis vectors $|c\rangle$ and $\mid c^{\prime}>$ in $\Omega_{f}+\Omega_{f b}$ satisfies

$$
\left\langle c^{\prime}\left|\mathcal{H}_{1}\right| c\right\rangle=\left(c^{\prime}|h| c\right) \equiv W_{c^{\prime} c}
$$

From the lemma the equivalence theorem follows immediately: The finite energy sector of $H$ (spanned by the subspace $\Omega_{f}+\Omega_{f b}$ ) has an identical spectrum and an identical $S$-matrix as those of $h$ (spanned by the Hilbert space $\Omega_{h}$ ). The following model gives a concrete illustration for the construction of $\mathcal{H}_{1}$.

\subsection{Resonance-Boson Model}

Consider the example of a fermion system on a simple cubic lattice of sites $j=1,2, \cdot \cdot, \mathcal{N}$ and unit spacing $\ell ;$ in which the Hamiltonian $h$ is

$$
h=h_{0}+h_{g}+h_{f},
$$

with

$$
h_{0}=6 t \sum_{j} \alpha_{j \sigma}^{\dagger} \alpha_{j \sigma}-t \sum_{<i j\rangle}\left(\alpha_{i \sigma}^{\dagger} \alpha_{j \sigma}+\alpha_{j \sigma}^{\dagger} \alpha_{i \sigma}\right)
$$


Throughout the paper, the operators $\alpha_{j \sigma}$ and their hermitian conjugate satisfy (1.1), and a summation over the spin

$$
\sigma=\uparrow \quad \text { and } \quad \downarrow
$$

is to be carried out whenever $\sigma$ appears twice in a product, and the $\langle i j\rangle$ summation extends over all different pairs $\langle i j\rangle$ of nearest neighbors. (For a simple cubic lattice, there are $3 \mathcal{N}$ pairs of $<i j>$.) In (1.22), there are two interactions; one is a same-site attraction term

$$
h_{g}=-g \sum_{j} \alpha_{j \uparrow}^{\dagger} \alpha_{j \downarrow}^{\dagger} \alpha_{j \downarrow} \alpha_{j \uparrow},
$$

and the other gives a nearest-neighbor repulsion

$$
h_{f}=\frac{f}{4} \sum_{<i j>}\left(\alpha_{i \uparrow}^{\dagger} \alpha_{j \downarrow}^{\dagger}-\alpha_{i \downarrow}^{\dagger} \alpha_{j \uparrow}^{\dagger}\right)\left(\alpha_{j \downarrow} \alpha_{i \uparrow}-\alpha_{j \uparrow} \alpha_{i \downarrow}\right),
$$

with both $g$ and $f$ positive (except in Section 2.4, when we discuss the negative $f$ and/or $g$ cases). By construction, these interactions affect only the spin-singlet pairs.

The special case $f=0$ has been extensively studied ${ }^{2,3}$ : when $g$ is larger than a critical value

$$
\hat{g} \equiv \frac{g}{2 t}>3.9568
$$

the two-fermion sector has a singlet bound state, and the system becomes bosonic. For $g$ less than the critical value, there is no resonance state and the system is fermionic. For $f>0$, the situation is more complex: depending on the magnitudes of $g$ and $f$, there are three regions: (i) stable bosons, (ii) resonance bosons and (iii) neither. Of these three, case (ii) is perhaps physically the most interesting. This is because the resoriance boson model ${ }^{3-5}$ may provide an important key element towards the understanding of high $T_{c}$ superconductivity. However, that bosonic resonances can emerge from a simple single-band fermion Hamiltonian (1.22) has not received sufficient serious attention in the literature. The model Hamiltonian $h$ then provides both an illustration for the equivalence theorem and a model for resonance bosons.

A characteristic of any resonance-boson model is that the boson state (called $b$ ) is unstable in the two-fermion (called $2 e$ ) sector. The decay 


$$
b \rightarrow e+e
$$

for a boson at rest, carries an excitation energy $2 \nu$ with a width $\gamma$. When the charge density $n$ is small, there is no boson, except in virtual states. The system is superconducting at low temperatures, and its superconductivity is well described by the BCS theory. At a sufficiently high density, the bosons can be stabilized. This occurs when the Fermi energy $\epsilon_{F}$ reaches

$$
\epsilon_{F}=\nu
$$

which determines the first critical density $n=n_{1}$; the decay (1.28) becomes forbidden because of the exclusion principle. (There is some latitude in the definition of $n_{1}$ because of the width $\gamma$.) For $n>n_{1}$ the Fermi energy is essentially leveled at $\nu$, with the excess density $n-n_{1}$ fed into the boson channel. Both the bosons and the fermions are superconducting at low temperatures. The critical temperature $T_{c}$ is now dominated by the Bose-Einstein (BE) condensation, which is determined by having the thermal wavelength $\lambda_{T}$ of the bosons comparable to their inter-particle distance $d$. Because $\lambda_{T}$ is proportional to $\left(m_{b} T_{c}\right)^{-\frac{1}{2}}$, a small $m_{b}$ can lead to a high $T_{c}$. (For He II, $T_{c}$ is $2.12^{\circ} \mathrm{K}$, but $m_{b}$ is $4 \mathrm{GeV} / \mathrm{c}^{2}$ !) The BE feature is destroyed when the density becomes too large, and $d$ is smaller than the diameter of the boson. Thus, above a second critical density $n_{2}$, the system ceases to be a superconductor, a feature shared by all high $T_{c}$ superconductors.

For the equivalence theorem we consider, instead of the purely fermionic Hamiltonian $h$, a different system in which both fermionic and bosonic operators exist on each lattice site $j$, as described by (1.3). The Hamiltonian $\mathcal{H}=\lambda \mathcal{H}_{0}+\mathcal{H}_{1}$ of (1.5) for this new system is, in accordance with (1.19)-(1.20),

$$
H=H_{0}+H_{f}+H_{\text {core }}
$$

where $\lambda \mathcal{H}_{0}=H_{\text {core }}$ is a hard-core repulsion for particles on the same site, given by

$$
H_{\text {core }}=\sum_{j}\left(\xi_{0} a_{j \uparrow}^{\dagger} a_{j \downarrow}^{\dagger} a_{j \downarrow} a_{j \uparrow}+\eta_{0} a_{j \sigma}^{\dagger} b_{j}^{\dagger} b_{j} a_{j \sigma}+\frac{1}{4} \zeta_{0} b_{j}^{\dagger 2} b_{j}^{2}\right)
$$

with

$$
\xi_{0}=\lambda \hat{\xi}_{0}, \quad \eta_{0}=\lambda \hat{\eta}_{0} \text { and } \zeta_{0}=\lambda \hat{\zeta}_{0}
$$


so that as $\lambda \rightarrow \infty$, the three parameters $\xi_{0}, \eta_{0}$ and $\zeta_{0}$ all $\rightarrow \infty$. The Hamiltonian $\mathcal{H}_{1} \equiv H_{0}+H_{f}$ is given by

$$
\begin{aligned}
H_{0}= & 6 t \sum_{j} a_{j \sigma}^{\dagger} a_{j \sigma}-t \sum_{<i j>}\left(a_{i \sigma}^{\dagger} a_{j \sigma}+a_{j \sigma}^{\dagger} a_{i \sigma}\right) \\
& +(12 t-g) \sum_{j} b_{j}^{\dagger} b_{j}-t \sum_{<i j>}\left[\left(a_{i \uparrow}^{\dagger} a_{j \downarrow}^{\dagger}-a_{i \downarrow}^{\dagger} a_{j \uparrow}^{\dagger}\right)\left(b_{i}+b_{j}\right)+h . c .\right] \\
& +t \sum_{<i j\rangle}\left(b_{i}^{\dagger} a_{j \sigma}^{\dagger} a_{i \sigma} b_{j}+b_{j}^{\dagger} a_{i \sigma}^{\dagger} a_{j \sigma} b_{i}\right)
\end{aligned}
$$

and the nearest neighbor repulsive interaction,

$$
\begin{aligned}
H_{f}= & \frac{f}{4} \sum_{<i j>}\left[\left(a_{i \uparrow}^{\dagger} a_{j \downarrow}^{\dagger}-a_{i \downarrow}^{\dagger} a_{j \uparrow}^{\dagger}\right)\left(a_{j \downarrow} a_{i \uparrow}-a_{j \uparrow} a_{i \downarrow}\right)\right. \\
& \left.+2 b_{i}^{\dagger} b_{j}^{\dagger} b_{j} b_{i}+b_{i}^{\dagger} a_{j \sigma}^{\dagger} a_{j \sigma} b_{i}+b_{j}^{\dagger} a_{i \sigma}^{\dagger} a_{i \sigma} b_{j}\right] .
\end{aligned}
$$

As before, all repeated spin indices are to be summed over. It can be readily verified that (1.21) holds for any two basis vectors in the $4^{\mathcal{N}}$ dimensional Hilbert space of $h$, thereby fulfilling the condition for the equivalence. These two Hamiltonians $H$ and $h$ are quite different. However, because of the equivalence theorem, in the limit of $\xi_{0}, \eta_{0}$ and $\zeta_{0}$ all becoming $+\infty$, the finite energy sector of $H$ has an identical spectrum and an identical $S$-matrix as those of $h$. A striking feature is that this identity holds for arbitrary values of $g$ and $f$, independently of the stability of the bosons.

Both $H$ and $h$ conserve the total charge number $Q$. For $h$, we have $[h, Q]=$ 0 where

$$
Q=\sum_{j} \alpha_{j \sigma}^{\dagger} \alpha_{j \sigma}
$$

For $H$, the corresponding operator is

$$
Q=\sum_{j}\left(a_{j \sigma}^{\dagger} a_{j \sigma}+2 b_{j}^{\dagger} b_{j}\right)
$$

with $[H, Q]=0$. The $Q=2$ sector of $h$ is analysed in Section 2, and that of $H$ in Section 3, with their identical results explicitly demonstrated. In Section 4 we examine the $Q=3$ and 4 sectors. The many-body problem of the same model will be discussed in a separate paper. 
The techniques developed in this paper for the simple model can be readily generalized to systems with several bands and, thereby, achieve a wider class of physical applications. 


\section{TWO PARTICLE SECTOR OF $h$}

\subsection{Spin-singlet Scattering State}

Let $|0\rangle$ be the vacuum state, and $\left|\vec{p}_{1}, \vec{p}_{2}\right\rangle$ be the spin-singlet scattering state whose asymptotic solution consists of two fermions, with momenta

$$
\vec{p}_{1}=\frac{\vec{P}}{2}+\vec{p} \quad \text { and } \quad \overrightarrow{p_{2}}=\frac{\vec{P}}{2}-\vec{p}
$$

These momenta are restricted to the Brillouin zone. Since the total momentum $\vec{P}$ is conserved, we may write

$$
\left|\vec{p}_{1}, \vec{p}_{2}\right\rangle=\frac{1}{\sqrt{2}} \sum_{\vec{q}} F_{\vec{p}}(\vec{q} \mid \vec{p})\left(\alpha_{\frac{\vec{p}+\vec{q} \uparrow}{\dagger}}^{\dagger} \alpha_{\frac{\vec{p}}{2}-\vec{q} \downarrow}^{\dagger}-\alpha_{\frac{\vec{p}}{2}+\vec{q} \downarrow}^{\dagger} \alpha_{\frac{\vec{p}}{2}-\vec{q} \uparrow}^{\dagger}\right) \mid 0>
$$

where $\alpha_{\vec{k} \sigma}$ is related to the operator $\alpha_{j \sigma}$, introduced into (1.1), through

$$
\alpha_{j \sigma}=\mathcal{N}^{-\frac{1}{2}} \sum_{\vec{k}} \alpha_{\vec{k} \sigma} e^{i \vec{k} \cdot \bar{r}_{j}}
$$

with $\vec{r}_{j}$ the position vector of the $j^{\text {th }}$ site. In terms of $\alpha_{\vec{k} \sigma}$ and $\alpha_{\vec{k} \sigma}^{\dagger}$, the free Hamiltonian (1.23) becomes

$$
h_{0}=\sum_{\vec{k}} \epsilon_{\vec{k}} \alpha_{\vec{k}_{\sigma}}^{\dagger} \alpha_{\vec{k} \sigma}
$$

where

$$
\epsilon_{\vec{k}}=2 t \sum_{\alpha}\left(1-\cos k_{\alpha} \ell\right),
$$

and the sum goes over the three Cartesian components $k_{\alpha}$, with

$$
\alpha=1,2 \text { and } 3 \text {. }
$$

The time-independent Schroedinger equation

$$
h\left|\vec{p}_{1}, \vec{p}_{2}\right\rangle=E\left|\vec{p}_{1}, \vec{p}_{2}\right\rangle
$$


gives

$$
\left(\epsilon_{+}+\epsilon_{-}-E\right) F_{\vec{P}}(\vec{q} \mid \vec{p})=\mathcal{N}^{-1} \sum_{\vec{k}}\left(g-f \sum_{\alpha} \cos q_{\alpha} \ell \cos k_{\alpha} \ell\right) F_{\vec{P}}(\vec{k} \mid \vec{p})
$$

where

$$
\epsilon_{ \pm}=\epsilon_{\frac{\beta}{2} \pm \vec{q}}
$$

When $\mathcal{N} \rightarrow \infty$, the scattering solution for an outgoing wave is

$$
2 F_{\vec{p}}(\vec{q} \mid \vec{p})=\delta_{\vec{q}, \vec{p}}+\delta_{\vec{q},-\vec{p}}+2 \mathcal{N}^{-1} \frac{(\vec{q}|\gamma(E, \vec{P})| \vec{p})}{E-\epsilon_{+}-\epsilon_{-}+i 0+}
$$

with

$$
E=\epsilon_{\overrightarrow{p_{1}}}+\epsilon_{\bar{p}_{2}}
$$

The derivation of the scattering amplitude $(\vec{q}|\gamma(E, \vec{P})| \vec{p})$ follows closely the analysis given in Reference 1 . The result can be conveniently expressed in terms of the $4 \times 1$ column matrix

$$
u(\vec{q})=\left(\begin{array}{c}
1 \\
\cos q_{1} \ell \\
\cos q_{2} \ell \\
\cos q_{3} \ell
\end{array}\right)
$$

its transpose $\tilde{u}(\vec{q})$, the $4 \times 4$ symmetric matrix $\mathcal{G}=\left(\mathcal{G}_{\mu \nu}\right)$ defined by

$$
\mathcal{G}_{\mu \nu}(E, \vec{P})=\frac{2 t}{\mathcal{N}} \sum_{\vec{q}} \frac{u_{\mu}(\vec{q}) u_{\nu}(\vec{q})}{\epsilon_{+}+\epsilon_{-}-E-i 0+}
$$

with $\epsilon_{ \pm}$given by (2.9), $u_{\mu}(\vec{q})$ the $\mu^{\text {th }}$ element of $u(\vec{q})$, and the $4 \times 4$ diagonal matrix $\mathcal{K}$ whose diagonal elements are

$$
\mathcal{K}=\frac{1}{2 t}\left(\begin{array}{cccc}
-g & & & \\
& f & & \\
& & f & \\
& & & f
\end{array}\right)
$$

Both $\mathcal{G}$ and $\mathcal{K}$ are dimensionless. We find

$$
(\vec{q}|\gamma(E, \vec{P})| \vec{p})=2 t \tilde{u}(\vec{q}) \mathcal{K}(1+\mathcal{G} \mathcal{K})^{-1} u(\vec{p})
$$


which is symmetric in $\vec{q}$ and $\vec{p}$. In (2.13), the matrix index $\mu$ (or $\nu$ ) will be labeled $0,1,2$, and 3 , so that $u_{0}(\vec{q})=1, u_{1}(\vec{q})=\cos q_{1} \ell$, etc. There are two useful formulas for $\mathcal{G}_{\mu \nu}(E, \vec{P})$ :

$$
(6-\hat{E}) \mathcal{G}_{00}(E, \vec{P})-1=2 \sum_{\alpha} \cos \left(P_{\alpha} \ell / 2\right) \mathcal{G}_{\alpha 0}(E, \vec{P})
$$

and

$$
(6-\hat{E}) \mathcal{G}_{\alpha 0}(E, \vec{P})=2 \sum_{\beta} \cos \left(P_{\beta} \ell / 2\right) \mathcal{G}_{\alpha \beta}(E, \vec{P})
$$

where the sum over $\alpha$ (and $\beta$ ) is in accordance with (2.6) and

$$
\hat{E}=\frac{E}{2 t} \text {. }
$$

When the total momentum $\vec{P}=0$, the cubic symmetry requires

$$
\mathcal{G}_{01}=\mathcal{G}_{02}=\mathcal{G}_{03}, \quad \mathcal{G}_{12}=\mathcal{G}_{23}=\mathcal{G}_{31} \text { and } \mathcal{G}_{11}=\mathcal{G}_{22}=\mathcal{G}_{33} \text {. }
$$

The inversion of the $4 \times 4$ matrix $(1+\mathcal{G} \mathcal{K})$ simplifies. As in Reference 1 , we derive for the scattering amplitude:

$$
(\vec{q}|\gamma(E, 0)| \vec{p})=\frac{X}{Z}+Y
$$

where, in terms of the dimensionless parameters $\hat{E}$,

$$
\begin{gathered}
\hat{g}=\frac{g}{2 t} \quad \text { and } \quad \hat{f}=\frac{f}{2 t} \\
X=(12 t / \hat{f})\left\{-\hat{g}\left[2+\hat{f}(6-\hat{E}) \mathcal{G}_{01}\right]+2 \hat{g} \hat{f} \mathcal{G}_{01} \sum_{\alpha}\left(\cos q_{\alpha} \ell+\cos p_{\alpha} \ell\right)\right. \\
\left.+\frac{2}{3} \hat{f}\left(1-\hat{g} \mathcal{G}_{00}\right)\left(\sum_{\alpha} \cos q_{\alpha} \ell\right)\left(\sum_{\beta} \cos p_{\beta} \ell\right)\right\} \\
Y=\frac{\hat{f}}{3}\left[1+\hat{f}\left(\mathcal{G}_{11}-\mathcal{G}_{12}\right)\right]^{-1}\left(2 \sum_{\alpha} \cos q_{\alpha} \ell \cos p_{\alpha} \ell-\sum_{\alpha \neq \beta} \cos q_{\alpha} \ell \cos p_{\beta} \ell\right)
\end{gathered}
$$

and

$$
Z=\left(\hat{E}-e_{0}\right)+\left(\hat{E}-e_{+}\right)\left(\hat{E}-e_{-}\right) \mathcal{G}_{00}
$$


with $e_{0}$ and $e_{ \pm}$given by

$$
e_{0}=6-\hat{g}-(12 / \hat{f})
$$

and

$$
e_{ \pm}=6-\frac{\hat{g}}{2} \pm \frac{\hat{g}}{2}[1+(48 / \hat{f} \hat{g})]^{\frac{1}{2}}
$$

therefore.

$$
e_{+}+e_{-}=12-\hat{g}
$$

and

$$
e_{+} e_{-}=6(6-\hat{g})-(12 \hat{g} / \hat{f})
$$

Because $[1+(48 / \hat{f} \hat{g})]^{\frac{1}{2}} \leq 1+(24 / \hat{f} \hat{g})$.

$$
e_{0} \leq e_{-}
$$

always.

As will be shown in Appendix A, the first term on the right-hand side of (2.19), $X / Z$, represents an $s$-wave state (i.e., a state realizing the unit representation of the cubic group) and the second term, $Y$, denotes a $d$-wave state (i.e., a state realizing the doublet representation of the cubic group); the corresponding phase shifts for these two partial waves are given by the expressions:

$$
e^{2 i \delta_{e}(E)}=\frac{\hat{E}-e_{0}+\left(\hat{E}-e_{+}\right)\left(\hat{E}-e_{-}\right) \mathcal{G}_{00}^{*}(E)}{\hat{E}-e_{0}+\left(\hat{E}-e_{+}\right)\left(\hat{E}-e_{-}\right) \mathcal{G}_{00}(E)}
$$

and

$$
e^{2 i \delta_{d}(E)}=\frac{1+\hat{f}\left(\mathcal{G}_{11}^{*}(E)-\mathcal{G}_{12}^{*}(E)\right)}{1+\hat{f}\left(\mathcal{G}_{11}(E)-\mathcal{G}_{12}(E)\right)}
$$

with $\mathcal{G}_{\mu \nu}(E)$ denoting $\mathcal{G}_{\mu \nu}(E, \vec{P})$ at $\vec{P}=0$.

\subsection{Bound State}

In order to have a bound state, the analytical continuation of the scattering amplitude $(\vec{q}|\gamma(E, \vec{P})| \vec{p})$ must have a pole at a real and negative $E$. In this section, we discuss only the case $\vec{P}=0$; the analysis for $\vec{P} \neq 0$ will be given in Section 3. From (2.19), we see that the $s$-state bound state pole can arise only when $Z=0$; i.e., because of (2.23), when ${ }^{3}$ 


$$
\mathcal{G}_{\infty 0}^{-1}=\frac{\left(e_{+}-\hat{E}\right)\left(e_{-}-\hat{E}\right)}{e_{0}-\hat{E}} \equiv I(E)
$$

where, on account of (2.24)-(2.26), $I(E)$ can also be written as

$$
I(E)=-\hat{E}+6+\frac{12}{\hat{f}}+\left(\frac{12}{\hat{f}}\right)^{2} \frac{1}{e_{0}-\hat{E}} .
$$

For $E$ negative, we may remove the $i 0+$ term in (2.13). The function $\mathcal{G}_{00}$ becomes real; it can be expressed as an average of

$$
\lambda \equiv\left[-\hat{E}+\left(\epsilon_{\vec{q}} / t\right)\right]^{-1}
$$

over the $\mathcal{N}$ sites in the momentum space, with

$$
\mathcal{G}_{00}=\langle\lambda\rangle \equiv \frac{1}{\mathcal{N}} \sum_{\vec{q}} \lambda .
$$

The derivative of $I(E)-\mathcal{G}_{00}^{-1}$ with respect to $\hat{E}=E / 2 t$ is

$$
\left.-1+\left(\frac{12}{\hat{f}}\right)^{2} \frac{1}{\left(e_{0}-\hat{E}\right)^{2}}+\frac{\left\langle\lambda^{2}\right\rangle}{\langle\lambda\rangle^{2}}\right\rangle 0,
$$

since the average $\left\langle\lambda^{2}\right\rangle$ is always larger than $\langle\lambda\rangle^{2}$.

When $\hat{E} \rightarrow \pm \infty, \mathcal{G}_{\infty} \rightarrow-\hat{E}^{-1}-6 \hat{E}^{-2}+\cdots$,

$$
\mathcal{G}_{00}^{-1} \rightarrow-\hat{E}+6+O\left(\hat{E}^{-1}\right)
$$

and therefore (assuming $f>0$ )

$$
I(E)-\mathcal{G}_{00}^{-1} \rightarrow \frac{12}{\hat{f}}>0 .
$$

Consequently, on account of (2.31) and (2.34), in order to have a negative $E$ satisfying (2.30), $I(E)$ must have a singularity along the negative $E$-axis, which implies

$$
e_{0}<0
$$


In this case, as $\hat{E}$ varies from $-\infty$ towards 0 , the function $I(E)-\mathcal{G}_{\infty}^{-1}$ increases from $\frac{12}{\hat{f}}$ at $\hat{E}=-\infty$ to $+\infty$ at $\hat{E}=e_{0}-$; it then jumps to $-\infty$ at $\hat{E}=e_{0}+$. and continues to increase with $\hat{E}$. At $\hat{E}=0$.

$$
\mathcal{G}_{00}=g_{0} \equiv \sum_{\bar{q}} \frac{1}{2 \mathcal{N}}\left(3-\sum_{\alpha} \cos q_{\alpha} \ell\right)^{-1}=0.25273
$$

The constant $g_{0}$ can also be expressed ${ }^{6}$ in terms of the elliptic integral:

$$
g_{0}=\frac{2}{\pi^{2}}(18+12 \sqrt{2}-10 \sqrt{3}-7 \sqrt{6}) K^{2}((2-\sqrt{3})(\sqrt{3}-\sqrt{2}))
$$

where

$$
K(k)=\int_{0}^{\frac{\pi}{2}}\left(1-k^{2} \sin ^{2} \phi\right)^{-\frac{1}{2}} d \phi .
$$

Thus, in order to have a zero for $I(E)-\mathcal{G}_{00}^{-1}$ at a negative $E$, we must have, in addition to (2.37), a positive $I(E)-\mathcal{G}_{\infty}^{-1}$ at $E=0$. Writing

$$
I_{0} \equiv \frac{e_{+} e_{-}}{e_{0}}
$$

for $I(0)$, we require, as the condition for a bound state,

$$
I_{0} \geq g_{0}^{-1}
$$

i.e.,

$$
\frac{6(6-\hat{g})-(12 \hat{g} / \hat{f})}{6-\hat{g}-(12 / \hat{f})} \geq 3.9568
$$

in which the equality sign holds when the binding energy is zero. Combining (2.37) with (2.41)-(2.42), we see that $e_{-}<0$. When $\hat{f}=0,(2.43)$ reduces to (1.27).

It is instructive to plot $12 / \hat{f}$ versus $\hat{g}$ at a fixed $I_{0}$. Equation (2.41) for different $I_{0}$ gives a family of hyperbolas:

$$
\left(\frac{12}{\hat{f}}+6-I_{0}\right)\left(\hat{g}-I_{0}\right)=\left(6-I_{0}\right)^{2} .
$$

It is remarkable that, for any $I_{0}$, at least one branch of the hyperbola passes through the same point 


$$
\hat{g}=6 \text { and } \frac{12}{\hat{f}}=0
$$

with the same slope -1 , as shown in Figure 1 . The area to the right of the hyperbola $I_{0}=g_{0}^{-1}$ (solid curve) and above $12 / \hat{f}=0$ in the upper right quadrant of the figure denotes the region in which the bound state exists, as indicated by the slanted shaded region. (Here, we are concerned only with the case $\hat{f}>0$; for $\hat{f}<0$, the limiting value (2.36) is no longer positive, and the condition for a bound state is altered, as will be discussed below in Section 2.4.)

\subsection{Poles on the Unphysical Sheet}

Continuing the discussion for the case $\vec{P}=0$ and $g, f$ both positive, we note that for $E$ real and positive, $\mathcal{G}_{00}$ is given by

$$
\mathcal{G}_{00}=\frac{2 t}{\mathcal{N}} \sum_{\vec{q}} \frac{1}{2 \epsilon_{\vec{q}}-E-i 0 t} .
$$

Thus, as $\mathcal{N} \rightarrow \infty$, there is a cut along the real axis from $E=0$ to $E_{\max }$, determined by the boundary of the Brillouin zone. The physical scattering region lies infinitesimally above the cut. In Appendix $B$, we prove that, except for the real axis, the scattering amplitude is analytic in the entire $E$ plane (called the physical sheet). To trace the resonance boson state, we must make the appropriate analytical continuation to the unphysical sheet.

As $\mathcal{N} \rightarrow \infty .(2.46)$ can be written as

$$
\mathcal{G}_{00}=\frac{\ell^{3}}{8 \pi^{3}} \int d^{3} q \rho(\vec{q}) \frac{2 t}{2 \epsilon_{\vec{q}}-E-i 0+}
$$

where $\rho(\vec{q})$ denotes the phase space density. For $E$ near zero, the analytical behavior of $\mathcal{G}_{00}$ is dominated by the behavior of $\epsilon_{\vec{q}}$ and $\rho(\vec{q})$ for small $\vec{q}$, which is the same as the continuum limit; that allows us to analytically continue $\mathcal{G}_{00}$ to the unphysical sheet (at least when $E$ is small).

In the following, in orcar to simplify our discussion, we assume the continuum approximation (replacing cubic symmetry by spherical symmetry):

$$
\epsilon_{\vec{q}}=t q^{2} \ell^{2}
$$


where $q=|\vec{q}|$ and

$$
\rho(\vec{q})= \begin{cases}1 & \text { if } 2 \epsilon_{\vec{q}}<E_{\max } \\ 0 & \text { otherwise }\end{cases}
$$

with $E_{\max }$ determined by the condition that $\mathcal{G}_{00}=g_{0}$ at $E=0$, as in (2.38). Define the variable $z$ by

$$
\hat{E}=\frac{E}{2 t} \equiv z^{2}
$$

(2.47) becomes

$$
\mathcal{G}_{00}=\frac{1}{4 \pi^{2}} \int_{-z_{m}}^{z_{m}} \frac{z^{2} d z^{\prime}}{z^{2}-z^{2}-i 0+}
$$

where

$$
z_{m}=\left(E_{\max } / 2 t\right)^{\frac{1}{2}}=2 \pi^{2} g_{0}=4.9887 .
$$

The continuum approximation (2.48)-(2.49) is valid only for $q$ small. Hence, the extension to the unphysical sheet (lower $z$-plane) for large $|z|$ is not reliable. Nevertheless, it provides a simple prototype, enabling us to gain insight into the kind of complications that can arise on the unphysical sheet. (In Appendix $C$, we give the analytical properties of $\mathcal{G}_{00}(E)$, together with a detailed analysis of the scattering amplitude poles on the "second unphysical sheet", without any approximations.)

From the integral representation (2.51), we see that $\mathcal{G}_{00}$ as a function of $z$ has a cut along the real axis from $z=-z_{m}$ to $+z_{m}$. Regard $z$ and $\hat{E}$ both as complex variables. When transformed from $z$ to $\hat{E}$, the upper half $z$-plane maps onto the entire physical $E$-plane. For $z$ real and greater than $z_{m}, \mathcal{G}_{00}$ is real. For $z$ real between $-z_{m}$ and $z_{m}$, but infinitesimally above the cut, $\mathcal{G}_{00}$ acquires an imaginary part $i \frac{z}{4 \pi}$ :

$$
\mathcal{G}_{00}=g_{0}+\frac{z}{4 \pi^{2}}\left(\ln \left|\frac{z_{m}-z}{z_{m}+z}\right|+i \pi\right),
$$

in which the logarithm is real.

To make the analytic continuation of $z$ into the regions $\operatorname{lm} z<0$ and $-z_{m}<$ $\operatorname{Re} z<+z_{m}$, we define the function

$$
\ln \left(\frac{z_{m}-z}{z_{m}+z}\right)
$$

to be real for $z$ real between $-z_{m}$ and $+z_{m}$, and impose two cuts along the real $z$-axis, one from $-\infty$ to $-z_{m}$ and the other from $+z_{m}$ to $+\infty$ (as shown by the 
wavy lines in Figure 2). Thus, (2.54) acquires an imaginary part above these cuts (e.g., its imaginary part is $-i \pi$ above the cut from $z=+z_{m}$ to $+\infty$ ). In terms of (2.54), introduce

$$
\mathcal{G}(z) \equiv g_{0}+\frac{z}{4 \pi^{2}}\left(\ln \frac{z_{m}-z}{z_{m}+z}+i \pi\right) .
$$

Clearly, for $\operatorname{Im} z=0+$

$$
\mathcal{G}(z)=\mathcal{G}_{\infty}
$$

along the entire real $z$-axis. Since $\mathcal{G}(z)$ is analytic in the region $\operatorname{Re} z$ between $-z_{m}$ and $z_{m}$, the analytic continuation to the unphysical sheet with $\operatorname{Im} z<0$ becomes easy.

Now, when mapped from $z$ to $\hat{E}$ through (2.50), the lower half $z$-plane be comes the entire second unphysical $E$-sheet. The continuum approximation (2.48)(2.49) enables us to trace explicitly the trajectory of the analytical continuation of the bound state pole' on the unphysical sheet when $I_{0}$ is less than $g_{0}^{-1}$; i.e., the bound state condition (2.42) is violated.

The pole and its analytical continuation are defined to be the solutions of

$$
\mathcal{G}(z)^{-1}=\frac{\left(e_{+}-z^{2}\right)\left(e_{-}-z^{2}\right)}{e_{0}-z^{2}} \equiv I(z)
$$

in the $z=x+i y$ plane. As in (2.31). $I(z)$ can also be written as

$$
I(z)=-z^{2}+6+\frac{12}{\hat{f}}+\left(\frac{12}{\hat{f}}\right)^{2} \frac{1}{e_{0}-z^{2}} .
$$

In the following, we shall show that: (i) When $\hat{f}=0$ and $\hat{g}$ is sufficiently large, (2.57) has no solution at any finite $z$ in the lower half-plane, whereas there is a bound state pole in the upper half-plane, in accordance with (1.27). (ii) With increasing $\hat{f}$ or decreasing $\hat{g}$, poles can enter the lower half-plane, but only along the negative $y$-axis, either from above (the physical sheet), or from below ( $y=$ $-\infty$ ). (iii) When $\hat{f}=0$, the latter does not happen and there is only one pole

$$
z=i y_{1}
$$

along the $y$-axis: for $\hat{g}>g_{0}^{-1}, y_{1}>0$ (bound state) and for $\hat{g}<g_{0}^{-1}, y_{1}<0$, which corresponds to neither resonance nor bound state. (iv) For $\hat{f}>0$, there 
are two solutions of (2.57): for $I_{0}=\frac{e_{+} e_{-}}{e_{0}}>g_{0}^{-1}$, both are along the $y$-axis, one with $y=y_{1}>0$ denoting the bound state and the other $y=y_{2}<0$, as shown in Figure 2. By gradually decreasing $I_{0}$ to less than $g_{0}^{-1}$, we find $y_{1}$ and $y_{2}$ moving towards each other and both becoming negative. When $I_{0}$ reaches a critical value $I_{c}$, between $g_{0}^{-1}$ and 0 , these two poles coalesce. In Figure 1 , the dotted curve refers to the critical trajectory $I_{0}=I_{c}$; its explicit form will be given below.

(v) For $I_{0}<I_{c}$, the two poles form a pair $z= \pm x_{r}+i y_{r}$. Both remain within the lower $z$-plane, and are the only poles there. The one with $x_{r}>0$ becomes the pole for the resonance boson. Depending on its location, the resonance can be sharp or broad.

To establish these intricate patterns, we list some properties satisfied by $\mathcal{G}(z)$ and $I(z)$. Write in (2.55),

$$
z_{m}-z=\left|z_{m}-z\right| e^{i \alpha_{1}}
$$

and

$$
z_{m}+z=\left|z_{m}+z\right| e^{-i \alpha_{2}}
$$

both can be represented by vectors in the complex $z$-plane (as shown by the two dashed lines in Figure 2). We find

$$
\operatorname{lm} \mathcal{G}(z)=\frac{y}{4 \pi^{2}} \ln \left|\frac{z_{m}-z}{z_{m}+z}\right|+\frac{x}{4 \pi^{2}}\left(\pi+\alpha_{1}+\alpha_{2}\right)
$$

and

$$
-y \operatorname{Re} \mathcal{G}(z)+x \operatorname{lm} \mathcal{G}(y)=-y g_{0}+\frac{x^{2}+y^{2}}{4 \pi^{2}}\left(\pi+\alpha_{1}+\alpha_{2}\right)
$$

In the lower half $z$-plane, because $y<0, \alpha_{1}$ and $\alpha_{2}$ are between 0 and $\pi$, each term on the right side of $(2.62)$ is positive definite, $\mathcal{G}(z) \neq 0$; this and that at $z=0, \mathcal{G}(z)=g_{0}$, even though (2.62) vanishes, imply

$$
\mathcal{G}(z)^{-1} \quad \text { is bounded }
$$

Furthermore, since $\ln \left|\frac{z_{m}-z}{z_{m}+z}\right|$ is odd in $x$, it follows from (2.61) that, for $x \neq 0$ and $y<0, \operatorname{lm} \mathcal{G}(z)^{-1}$ is also odd in $x$, with

$$
x\left[\operatorname{lm} \mathcal{G}(z)^{-1}\right] \leq 0
$$


By using (2.24) and (2.58), we find as $\hat{f} \rightarrow 0, I(z) \rightarrow \hat{g}$ at all finite $z$. This and (2.63) lead to the property (i) mentioned above. To prove (ii), we draw the contour $C=C_{1}+C_{2}$, shown in Figure 2, where $C_{1}$ is a straight line along $y=0$ - from $x=-R$ to $R$, and $C_{2}$ is a half circle of radius $R$, with $R$ approaching infinity in the end. From (2.58) and (2.61), we see that $\operatorname{Im} I(z)$ is zero along $C_{1}$, but $\operatorname{Im} \mathcal{G}(z)$ is not, except at $x=0$. Since $\operatorname{Im} I(z)=-2 x y\left[1-(12 / \hat{f})^{-2}\left|e_{0}-z^{2}\right|^{-2}\right]$, for $R^{2}>\left|e_{0}\right|+(12 / \hat{f})$, along $C_{2}, \operatorname{Im} I(z)$ is odd in $x$ but

$$
x[\ln I(z)] \geq 0
$$

which carries an opposite sign from (2.64). Thus, along the contour $C$, except at its intersections with the negative $y$-axis, the pole equation (2.57) has no solution; (ii) then follows.

To establish (iii) and (iv), we need only examine $\mathcal{G}(z)$ and $I(z)$ along the $z=i y$ axis: both are real with

$$
\mathcal{G}(i y)=g_{0}-\frac{y}{4 \pi^{2}}\left(\pi+\alpha_{1}+\alpha_{2}\right)
$$

and

$$
I(i y)=y^{2}+6+\frac{12}{\hat{f}}+\left(\frac{12}{\hat{f}}\right)^{2} \frac{1}{e_{0}+y^{2}} .
$$

In Figure 3, the dashed curve is $\mathcal{G}^{-1}$ vs. $y$, and the solid curves are $I$ vs. $y$ for different values of $\hat{f}$ and $\hat{g}$. Figure $3(a)$ refers to $\hat{f}=0$, and $I=\hat{g}$ is a straight line. Since $\mathcal{G}^{-1}=g_{0}^{-1}$ at $y=0$, there is only one intersection point which is at $y=y_{1}>0$ if $\hat{g}>g_{0}^{-1}$, and at $y=y_{1}<0$ if $\hat{g}<g_{0}^{-1}$. Thus, (iii) is proved.

For $\hat{f} \neq 0$ (and finite $\hat{g}$ ), the parameters $e_{0}$ and $e_{ \pm}$are all finite, and the dependence of $I(i y)$ on $y$ is more complicated. From (2.24)-(2.26), (2.36) and (2.66), it follows that

$$
\begin{gathered}
\frac{\partial \ln I_{0}}{\partial \hat{g}}=\frac{(12 / \hat{f})^{2}}{e_{+} e_{-} e_{0}} \\
\frac{\partial \ln I_{0}}{\partial(12 / \hat{f})}=\frac{(6-\hat{g})^{2}}{e_{+} e_{-} e_{0}}
\end{gathered}
$$

$\partial I(i y) / \partial y=0$ at 


$$
y^{2}=0 \quad \text { and } \quad-e_{0} \pm(12 / \hat{f})
$$

and at $y=0$

$$
\partial^{2} I(i y) / \partial y^{2}=\frac{2}{e_{0}^{2}}\left[e_{0}^{2}-\left(\frac{12}{\hat{f}}\right)^{2}\right]
$$

To appreciate the different shapes of $I(i y)$ vs. $y$, we start from the upper right extreme of Figure 1, with $e_{0}, e_{-}$both negative and $I_{0}$ positive and large; we then move towards the origin along the $\hat{g}=12 / \hat{f}$ line. Before crossing the $\hat{g}=6$ vertical line (on which $e_{0}=-12 / \hat{f}$ ), the curvature $\left(\partial^{2} I(i y) / \partial y^{2}\right)$ is positive at $y=0$, and a typical example of $I(i y)$ is given in Figure 3(b). There are two solutions of

$$
\mathcal{G}(i y)^{-1}=I(i y)
$$

at $y=y_{1}$ and $y_{2}$. For $y>0$, there is at most one solution, as already proved in the previous section (see (2.34)-(2.36)). In this example, we have $y=y_{1}$ for the bound state. After crossing the $\hat{g}=6$ line, $\partial^{2} I(i y) / \partial y^{2}$ becomes negative at $y=0$. As we move along the $\hat{g}=12 / \hat{f}$ line towards the origin in Figure 1 , $I_{0}$ decreases steadily and the two solutions $y_{1}$ and $y_{2}$ move towards each other, both negative after crossing the $I_{0}=g_{0}^{-1}$ hyperbola (end of the bound state), as illustrated in Figure 3(c). If we decrease $I_{0}$ further to $I_{c}$, the two curves $\mathcal{G}(i y)^{-1}$ and $I(i y)$ become tangent at $y=y_{c}$. It is clear that $I_{c}$ is between $g_{0}^{-1}$ and 0 .

Introduce

$$
\theta=\tan ^{-1}\left(-z_{m} / y_{c}\right)
$$

as shown in Figure 2, where at $y=y_{c}$, both (2.70) and

$$
\partial \ln I(i y) / \partial y=-\partial \ln \mathcal{G}(i y) / \partial y
$$

are satisfied. It can readily be verified that the critical trajectory $I_{0}=I_{c}$ (the dotted curve in Figure 1) is given by

$$
\frac{12}{\hat{f}}=\frac{P(\theta)}{1-Q(\theta)}
$$

and

$$
\hat{g}=6+4 \pi^{4} g_{0}^{2} \cot ^{2} \theta+\frac{P(\theta)}{Q(\theta)}
$$


where

$$
P(\theta)=\left[g_{0}(1+(\pi-\theta) \cot \theta)\right]^{-1}-4 \pi^{4} g_{0}^{2} \cot ^{2} \theta-6
$$

and

$$
Q(\theta)=\left[1-\frac{\tan \theta}{8 \pi^{4} g_{0}^{3}} \frac{\theta-\pi-\frac{1}{2} \sin 2 \theta}{(1+(\pi-\theta) \cot \theta)^{2}}\right]^{\frac{1}{2}}
$$

The aforementioned (iv) is then established. For $I_{0}<I_{c}$, the poles leave the negative $y$-axis, and one of them becomes the resonance pole, as described by $(v)$.

\subsection{Resonance-Boson}

As mentioned before, the "continuum approximation" (2.48)-(2.49) is not valid for large $|z|$, even though it does give the correct qualitative picture of the polebehavior on the second unphysical sheet. In this section, we shall discuss the resonance pole without approximations. All equations up to $(2.47)$ remain valid. The integral representation (2.47) defines $\mathcal{G}_{00}$ on the physical sheet. Because the integration domain is limited by the Brillouin zone, with

$$
\epsilon_{\vec{q}} \leq 12 t
$$

(2.47) shows that $\mathcal{G}_{00}(E)$ has cuts from $E=0$ to

$$
E_{\max }=24 t
$$

with the physical region lying on the real axis for $E>E_{\max }$, and infinitesimally above the real axis (i.e., slightly above the cut) for $E_{\max }>E>0$. As in (2.50), we define $z^{2}=\hat{E}=E / 2 t$; the upper half $z$-plane corresponds to the physical $E$-sheet and the lower half $z$-plane becomes the second unphysical $E$-sheet. The lower right quadrant of the $z$-plane is of particular interest since it corresponds to the lower half plane of the second unphysical $E$-sheet, which borders on the physical region.

Figure 1 (except for the dotted curve) is valid without any approximation. For $\hat{f}>0$ and $I_{0}=\frac{e_{+} e_{-}}{e_{0}}>g_{0}^{-1}$, there is a bound state at $z=i y_{1}$ with $y_{1}>0$. When $I_{0}=g_{0}^{-1}$, the bound state pole is at $y_{1}=0$. If we gradually decrease $I_{0}$, this pole will move along the imaginary $z=i y$ axis, with $y_{1}<0$, until $I$ reaches a critical value $I_{c}<g_{0}^{-1}$. At that point it will coalesce with another pole, originally located at $z=i y_{2}$ with $y_{2}<y_{1}$. For $I>I_{c}$, these two poles form a 
pair at $z= \pm x_{r}+i y_{r}$. The one with $x_{r}>0$ will be called the resonance pole (as in (v) of Section 2.3). In Figure 4, we plot the trajectory of the resonance pole and its conjugate pair on the $z$-plane as a function of $12 / \hat{f}$ for a fixed $\hat{g}=5$. The imaginary part denotes the width. The coalescence occurs at $\hat{f}=\hat{f}_{c} \cong 6$. For $\hat{f}>\hat{f}_{c}, x_{r}>0$ and $y_{r}$ decreases with increasing $\hat{f}: y_{r}=0$ as $\hat{f} \rightarrow \infty$. as indicated by the solid line in Figure 4 . (When $\hat{f}=\infty$, the potential barrier is infinite, and therefore the resonance width becomes zero.)

The location of the resonance pole at large $\hat{f}$ can readily be determined. (See Appendix $C$ for further exact properties of scattering amplitude poles without assuming $\hat{f}$ large.) It is convenient to rewrite (2.30) as:

$$
\hat{E}=6-\hat{g}-\frac{12}{\hat{f}}-\left(\frac{12}{\hat{f}}\right)^{2} \frac{1}{\hat{E}-6-\frac{12}{\hat{f}}+\mathcal{G}_{00}^{-1}(E)} .
$$

Through iteration we can obtain its solution, $E=E_{\text {pole }}$, as

$$
E_{\text {pole }}=2 t\left[6-\hat{g}-\frac{12}{\hat{f}}-\left(\frac{12}{\hat{f}}\right)^{2} \frac{\mathcal{G}_{00}(6-\hat{g})}{1-\hat{g} \mathcal{G}_{00}(6-\hat{g})}+O\left(\frac{1}{\hat{f}^{3}}\right)\right] \equiv E_{0}-\frac{i}{2} \Gamma
$$

where $\mathcal{G}_{00}(6-\hat{g})$ denotes $\mathcal{G}_{00}(E)$ at $E=2 t(6-\hat{g})$.

$E_{0}=\operatorname{Re} E_{\text {pole }}=2 t\left[6-\hat{g}-\frac{12}{\hat{f}}-\left(\frac{12}{\hat{f}}\right)^{2} \frac{\operatorname{Re} \mathcal{G}_{00}(6-\hat{g})-\hat{g}\left|\mathcal{G}_{00}(6-\hat{g})\right|^{2}}{\left|1-\hat{g} \mathcal{G}_{00}(6-\hat{g})\right|^{2}}+O\left(\frac{1}{\hat{f}^{3}}\right)\right]$

and the resonance half-width

$$
\frac{\Gamma}{2}=-\operatorname{Im} E_{\text {pole }}=2 t\left(\frac{12}{\hat{f}}\right)^{2} \frac{\operatorname{lm} \mathcal{G}_{00}(6-\hat{g})}{\left|1-\hat{g} \mathcal{G}_{00}(6-\hat{g})\right|^{2}}>0
$$

since

$$
\operatorname{lm} \mathcal{G}_{00}(E)=2 t \ell^{3} \int \frac{d^{3} \vec{p}}{(2 \pi)^{3}} \delta\left(2 \epsilon_{p}-E\right)>0
$$

for $0<E<24 t$.

In the limit $\hat{f} \rightarrow \infty$, the pole (2) is settled at $E_{\text {pole }}=12 t-g$ in the physical region of $E$. This pole corresponds to a real boson state of a positive energy (assuming $g<12 t$ ), but is stabilized by the infinite barrier of the nearest-neighbor repulsion. The two fermions in this spin-singlet state stay always together on the 
same lattice site. The residue of the scattering amplitude at this pole is equal to zero and therefore the scattering amplitude is finite there.

It is instructive to examine the relevance of the resonance pole at a large $\hat{f}$ to the behavior of the scattering phase shift within the physical domain of $E$. The phase shift (2.28) for the $s$-wave can be decomposed into two factors:

$$
e^{2 i \delta_{0}(E)}=e^{2 i \delta_{0}(E)} e^{2 i \delta_{1}(E)}
$$

with

$$
e^{2 i \delta_{0}(E)}=\frac{\left(\hat{E}-6+\hat{g}+\frac{12}{\hat{f}}\right)+\left(\frac{12}{f}\right)^{2}\left(\hat{E}-6-\frac{12}{\hat{f}}+\mathcal{G}_{00}^{-1}(E)^{*}\right)^{-1}}{\left(\hat{E}-6+\hat{g}+\frac{12}{f}\right)+\left(\frac{12}{f}\right)^{2}\left(\hat{E}-6-\frac{12}{f}+\mathcal{G}_{00}^{-1}(E)\right)^{-1}}
$$

and

$$
e^{2 i \delta_{1}(E)}=\frac{1+\left(\hat{E}-6-\frac{12}{\hat{f}}\right) \mathcal{G}_{00}^{*}(E)}{1+\left(\hat{E}-6-\frac{12}{\hat{f}}\right) \mathcal{G}_{00}(E)}
$$

For a narrow resonance $(\hat{f}>>1)$, the variation of the phase shift in the vicinity of $E_{0}$, the real part of the resonance pole, is dominated by the factor $e^{2 i \delta_{0}(E)}$, which takes the simple Breit-Wigner form approximately:

$$
e^{2 i \delta_{0}(E)} \cong \frac{E-E_{0}-\frac{i}{2} \Gamma}{E-E_{0}+\frac{i}{2} \Gamma}
$$

Therefore, $\delta_{0}(E)$ sweeps rapidly over a range $\cong \pi$ as $E$ varies from $E<E_{0}$ to $E>E_{0}$ over an interval of the order of $\hat{f}^{-2}$. As long as the factor $e^{2 i \delta_{1}(E)}$ stays away from -1 , there exists an $\bar{E}=E_{0}+O\left(\frac{1}{f^{2}}\right)$ such that the phase shift $\delta_{s}(\bar{E})=$ an odd multiple of $\pi / 2$, in accordance with a different, but often used criterion for a resonance. The value $\bar{E}$ is determined by the condition that the real part of the denominator and that of the numerator, which is complex conjugate to the denominator, of (2.28) should vanish at $\bar{E}$, i.e.

$$
\frac{1}{I(\bar{E})}=\operatorname{Re} \mathcal{G}_{00}(\bar{E})
$$

Following the same procedures leading from $(2.30)$ to $(2.78)-(2.79)$, we find

$$
\bar{E}=2 t\left[6-\hat{g}-\frac{12}{\hat{f}}-\left(\frac{12}{\hat{f}}\right)^{2} \frac{\operatorname{Re} \mathcal{G}_{00}(6-\hat{g})}{1-\hat{g} \operatorname{Re} \mathcal{G}_{00}(6-\hat{g})}+O\left(\frac{1}{\hat{f}^{3}}\right)\right]
$$


Consequently, these two definitions of resonance energy $\bar{E}$ and $E_{0}$ are the same only to $O(12 / \hat{f})$, when the width is narrow.

The above expressions (2.79)-(2.88) are also valid for $g<0$, provided that $f$ is positive and sufficiently large. (In this case, both the same-site and the nearestneighbor spin singlet interactions are repulsive.)

\subsection{Negative $f$ Region}

In this paper, we are mainly concerned with the case $g$ and $f$ both positive since, in accordance with (1.25)-(1.26), the same site attraction due to a positive $g$ gives the appropriate binding for either the bound state or the resonance; a positive $f$ provides the nearest neighbor repulsion, which acts as a potential barrier and serves as an essential element for the resonance-width. The bound state region is represented by the slanted shaded area in the upper right quadrant in Figure 1 (to the right of the upper branch of the hyperbola $I_{0}=g_{0}^{-1}$ and above the abscissa $12(\hat{f}=0)$. The resonance area is to the left of $I_{0}=I_{c}$, the dotted curve in Figure 1.

From Figure 1, one sees that the upper branch of the hyperbola $I_{0}=g_{0}^{-1}$ also extends to $\hat{f}<0$ (indicated by the vertically shaded region in Figure 1 ). To understand the physical implication of this region, we give a brief discussion of the negative $f$ area in this section.

For $f<0$, the nearest neighbor singlet interaction is attractive. Instead of the positive sign in (2.36), we have, as $\hat{E} \rightarrow \pm \infty$.

$$
I(E)-\mathcal{G}_{\infty}^{-1} \rightarrow \frac{12}{\hat{f}}<0
$$

On the other hand, (2.34) remains valid; therefore

$$
\frac{\partial}{\partial \hat{E}}\left[I(E)-\mathcal{G}_{\infty}^{-1}\right]>0
$$

along the entire negative $E$-axis. In Figure 1, the lower $(\hat{f}<0)$ half-plane may be classified into four different regions:
(a) $e_{0}>0$ and $I_{0}>g_{0}^{-1}$,
(b) $e_{0}<0$ and $I_{0}<g_{0}^{-1}$, 


$$
\text { (c) } e_{0}<0 \text { and } I_{0}>g_{0}^{-1} \text {, }
$$

and

$$
\text { (d) otherwise (i.e., } e_{0}>0 \text { and } I_{0}<g_{0}^{-1} \text { ). }
$$

In (a), the function $I(E)-\mathcal{G}_{\infty}^{-1}$ has no singularity along the negative $E$-axis; varying $\hat{E}=E / 2 t$ from $-\infty$ to 0 , we see that the function begins with a negative value, $12 / \hat{f}$, and then increases steadily to $I_{0}-g_{0}^{-1}>0$ at $\hat{E}=0$. Thus, there is one bound state.

For $e_{0}<0$, along the negative $E$-axis the function $I(E)-\mathcal{G}_{00}^{-1}$ becomes singular at $\hat{E}=e_{0}$. Varying $\hat{E}$ from $-\infty$ to 0 , we see that the function again starts from $12 / \hat{f}<0$ at $\hat{E}=-\infty$, then approaches $+\infty$ as $\hat{E}=e_{0}-$; hence, there is one zero (bound state) at $\hat{E}<e_{0}$. At $\hat{E}=e_{0}+$, the function jumps to $-\infty$, then continues to increase and reaches $I_{0}-g_{0}^{-1}$ at $\hat{E}=0$. Consequently, for (b), $I_{0}<g_{0}^{-1}$, there is no additional bound state between $\hat{E}=e_{0}$ and 0 . But for (c), $I_{0}>9_{0}^{-1}$, there is an additional bound state. $((b)$ is indicated by the horizontally shaded region in Figure 1, and (c) by the vertically shaded region.)

For (d), there is no bound state. Here, as before, we only examine the $s$-state singlet levels. 


\section{3. $Q=2$ SECTOR OF $H$}

The Hamiltonian $H$ given by $(1.30)$ is quite different from $h$. Let $\mid 0>$ be the vacuum state of $H$, satisfying

$$
a_{j \sigma} \mid 0>=0 \text { and } b_{j} \mid 0>=0
$$

for all $j$ and $\sigma$. Unlike (2.2), in the $Q=2$ sector of $H$, there are now two types of states

$$
a_{\vec{p}_{1} \sigma_{1}}^{\dagger} a_{\vec{p}_{2} \sigma_{2}}^{\dagger} \mid 0>=0 \text { and } b_{\vec{P}}^{\dagger} \mid 0>
$$

where $a_{\vec{k} \sigma}$ and $b_{\vec{k}}$ are related, in the same way as (2.3), to the operators $a_{j \sigma}$ and $b_{j}$, introduced in (1.3). In accordance with the equivalence theorem, the finite energy spectrum and the scattering matrix of $H$ become the same as those of $h$ when the hard core interaction $H_{\text {core }}$ ( $(1.31)$, becomes infinite (i.e., in the $Q=2$ sector, when the coupling constant $\left.\xi_{0} \rightarrow \infty\right)$, as we shall see.

In the following we rewrite $H_{0}$ of (1.32) as a sum of two terms

$$
H_{0}=H_{\text {free }}+H_{t}
$$

where

$$
H_{\text {free }}=\sum_{\vec{E}}\left(\epsilon_{\vec{k}} a_{\vec{k} \sigma}^{\dagger} a_{\vec{k} \sigma}+m_{0} b_{\vec{k}}^{\dagger} b_{\vec{k}}\right)
$$

and

$$
\begin{aligned}
H_{t}= & -t \sum_{<i j>}\left[\left(a_{i \uparrow}^{\dagger} a_{j \downarrow}^{\dagger}-a_{i \downarrow}^{\dagger} a_{j \uparrow}^{\dagger}\right)\left(b_{i}+b_{j}\right)+\text { h.c. }\right] \\
& +t \sum_{<i j>}\left(b_{i}^{\dagger} a_{j \sigma}^{\dagger} a_{i \sigma} b_{j}+b_{j}^{\dagger} a_{i \sigma}^{\dagger} a_{j \sigma} b_{i}\right)
\end{aligned}
$$

with

$$
m_{0}=12 t-g
$$

and $\epsilon_{\vec{k}}$ is given by (2.5). In the interaction representation with the zeroth order Hamiltonian given by $H_{\text {free }}$ and the interaction Hamiltonian being

$$
H_{\text {int }} \equiv H_{t}+H_{f}+H_{\text {core }}
$$

the relevant bare propagators and vertices for the $Q=2$ sectors are listed in Figure 5. The propagator for the fermion is $i\left(k_{0}-\epsilon_{\vec{k}}+i 0+\right)^{-1}$, represented by a solid 
line; that of the boson is $i\left(k_{0}-m_{0}+i 0+\right)^{-1}$, represented by a dashed line, where $k_{0}$ is the "virtual" energy variable, conjugate to time. All arrows are in the direction of charge-flow. The vertex for the scattering of two fermions of momentum-spin

$$
\left(\frac{\vec{P}}{2}+\vec{p}, \uparrow\right)+\left(\frac{\vec{P}}{2}-\vec{p}, \downarrow\right) \rightarrow\left(\frac{\vec{P}}{2}+\vec{q}, \uparrow\right)+\left(\frac{\vec{P}}{2}-\vec{q}, \downarrow\right)
$$

is

$$
\frac{-i}{\mathcal{N}} 2 t \tilde{u}(\vec{q}) \kappa u(\vec{p})
$$

with the $4 \times 4$ diagonal matrix

$$
\kappa \equiv \frac{1}{2 t}\left(\begin{array}{llll}
\xi_{0} & & & \\
& f & & \\
& & f & \\
& & & f
\end{array}\right)
$$

and $u(\vec{p})$ given by (2.12). The vertex for the transition between a boson (denoted by $b$ ) of momentum $\vec{P}$ to a fermion spin singlet-pair

is

$$
b \rightarrow\left(\frac{\vec{P}}{2}+\vec{p}, \uparrow\right)+\left(\frac{\vec{P}}{2}-\vec{p}, \downarrow\right)
$$

$$
\frac{i}{\sqrt{\mathcal{N}}} 4 t \sum_{\alpha} \cos \left(\frac{P}{2}\right)_{\alpha} \ell \cos p_{\alpha} \ell
$$

All the summation conventions are the same as before.

\subsection{Boson Propagator}

In the Hamiltonian $H$, both bosons and fermions represent independent degrees of freedom. The definition of the boson propagator, either the bare one or the dressed one, is unambiguous. To derive the dressed boson propagator, represented by a wavy line in Figure $6(\mathrm{~b})$, we need the sum $\hat{\Gamma}$ of all one-particle irreducible scattering diagrams of two fermions for the transition (3.8). Figure $6(a)$ gives the integral equation

$$
\begin{aligned}
& <\vec{q}\left|\hat{\Gamma}\left(P_{0}, \vec{P}\right)\right| \vec{p}>=2 t \tilde{u}(\vec{q}) \kappa u(\vec{p}) \\
& \quad+\frac{i}{\mathcal{N}} \sum_{\vec{k}} \int \frac{d k_{0}}{2 \pi} \frac{\left.2 t \tilde{u}(\vec{q}) \kappa u(\vec{k})<\vec{k}\left|\hat{\Gamma}\left(P_{0}, \vec{P}\right)\right| \vec{p}\right\rangle}{\left(\frac{P_{0}}{2}+k_{0}-\epsilon_{\frac{\beta}{2}+\vec{k}}+i 0+\right)\left(\frac{P_{0}}{2}-k_{0}-\epsilon_{\frac{\vec{P}}{2}-\vec{k}}+i 0+\right)}
\end{aligned}
$$


where $\vec{P}$ is the total momentum and $P_{0}$ the total "energy". In Figure 6(a), $\vec{p}_{1}+\vec{p}_{2}=\vec{q}_{1}+\vec{q}_{2}=\vec{P}, \vec{p}_{1}-\vec{p}_{2}=2 \vec{p}$ and $\vec{q}_{1}-\vec{q}_{2}=2 \vec{q}$. As in (2.15), this integral equation can readily be solved; the solution is

$$
\left\langle\vec{q}\left|\hat{\Gamma}\left(P_{0}, \vec{P}\right)\right| \vec{p}\right\rangle=2 t \tilde{u}(\vec{q}) \kappa(1+\mathcal{G} \kappa)^{-1} u(\vec{p})
$$

with $\mathcal{G}$ given by (2.13). (For the Hamiltonian $H$, the complete two-fermion scattering amplitude $\Gamma$ contains also one-particle reducible diagrams; $\Gamma \neq \hat{\Gamma}$, as will be discussed in the next section.)

Let $-i \Pi\left(P_{0}, \vec{P}\right)$ be the sum of all one-particle irreducible boson propagator diagrams, with the external lines amputated. It can be separated into two terms, $-i \Pi_{1}\left(P_{0}, \vec{P}\right)$ and $-i \Pi_{2}\left(P_{0}, \vec{P}\right)$. The former does not contain $\hat{\Gamma}$ and the latter does; they are given by the two diagrams on the right-hand side of Figure $6(\mathrm{c})$. We find

$$
\Pi_{1}=-2 t(6-\hat{E})\left[(6-\hat{E}) \mathcal{G}_{\infty}-1\right]
$$

and, by using (2.16) and (3.14),

$$
\Pi_{2}=2 t\left[(6-\hat{E})^{2} \mathcal{G} \kappa(1+\mathcal{G} \kappa)^{-1} \mathcal{G}+\kappa(1+\mathcal{G} \kappa)^{-1}(1-2(6-\hat{E}) \mathcal{G})\right]_{\infty}
$$

where

$$
\hat{E}=E / 2 t=P_{0} / 2 t
$$

and the subscripts on the right-hand side denote the 00 element of the $4 \times 4$ matrix inside the square bracket. In terms of

$$
\Pi\left(P_{0}, \vec{P}\right)=\Pi_{1}\left(P_{0}, \vec{P}\right)+\Pi_{2}\left(P_{0}, \vec{P}\right)
$$

the inverse of the dressed boson propagator is given by

$$
D^{-1}\left(P_{0}, \vec{P}\right)=-i\left[P_{0}-12 t+g-\Pi\left(P_{0}, \vec{P}\right)+i 0+\right]^{-1}
$$

We now take the limit $\xi_{0} \rightarrow \infty$. Consider the matrix

$$
\mathcal{G}+\kappa^{-1}
$$

At $\xi_{0}=\infty$, it becomes 


$$
\hat{\mathcal{F}}=\mathcal{G}+\hat{f}^{-1}\left(\begin{array}{llll}
0 & & & \\
& 1 & & \\
& & 1 & \\
& & & 1
\end{array}\right)
$$

where, as before, $\hat{f}=f / 2 t$, and the off-diagonal elements of a diagonal matrix are not exhibited. Since

$$
\hat{\mathcal{F}}_{\mu 0}=\mathcal{G}_{\mu 0}
$$

and

$$
\kappa(1+\mathcal{G} \kappa)^{-1}=\left(\mathcal{G}+\kappa^{-1}\right)^{-1},
$$

we have, in the limit $\xi_{0}=\infty$.

$$
\left[\kappa(1+\mathcal{G} \kappa)^{-1} \mathcal{G}\right]_{\infty 0}=\sum_{\mu=0}^{3} \hat{\mathcal{F}}_{0 \mu}^{-1} \mathcal{G}_{\mu 0}=1
$$

and

$$
\left[\mathcal{G} \kappa(1+\mathcal{G} \kappa)^{-1} \mathcal{G}\right]_{\infty}=\sum_{\mu, \nu} \mathcal{G}_{0 \mu} \hat{\mathcal{F}}_{\mu \nu}^{-1} \mathcal{G}_{\nu 0}=\mathcal{G}_{00}
$$

substituting these formulas into (3.15)-(3.19), we find that the boson propagator can be expressed as the ratio of two determinants:

$$
D\left(P_{0}, \vec{P}\right)=\frac{i \operatorname{det} \hat{\mathcal{F}}\left(P_{0}, \vec{P}\right)}{g \operatorname{det} \mathcal{F}\left(P_{0}, \vec{P}\right)}
$$

where

$$
\mathcal{F}=\mathcal{G}+\mathcal{K}^{-1}
$$

with $\mathcal{K}$ given by (2.14). When $g^{-1}=0, \mathcal{F}$ reduces to $\hat{\mathcal{F}}$.

In terms of the two $3 \times 1$ column matrices

$$
v \equiv\left(\begin{array}{l}
\mathcal{G}_{10} \\
\mathcal{G}_{20} \\
\mathcal{G}_{30}
\end{array}\right) \quad, \quad w \equiv\left(\begin{array}{c}
\cos \frac{p_{1}}{2} \ell \\
\cos \frac{P_{2}}{2} \ell \\
\cos \frac{P_{3}}{2} \ell
\end{array}\right)
$$

and the $3 \times 3$ symmetric matrix

$$
G \equiv\left(\mathcal{G}_{\alpha \beta}\right)
$$


with the subscripts $\alpha, \beta$ varying from 1 to $3,(2.16)$ becomes

$$
2 G w=(6-\hat{E}) v
$$

and

$$
2 \tilde{v} w=(6-\hat{E}) \mathcal{G}_{\infty}-1
$$

the matrices $\hat{\mathcal{F}}$ and $\mathcal{F}$ can be written as

$$
\hat{\mathcal{F}}=\left(\begin{array}{cc}
\mathcal{G}_{00} & \tilde{v} \\
v & F
\end{array}\right), \quad \mathcal{F}=\left(\begin{array}{cc}
\mathcal{G}_{00}-\hat{g}^{-1} & \tilde{v} \\
v & F
\end{array}\right)
$$

where $\hat{g}=g / 2 t$, as before, and $F$ is a $3 \times 3$ matrix given by

$$
F=G+\hat{f}^{-1}
$$

Substituting

$$
\begin{gathered}
\operatorname{det} \hat{\mathcal{F}}=\left(\mathcal{G}_{\infty}-\tilde{v} F^{-1} v\right) \operatorname{det} F, \\
\operatorname{det} \mathcal{F}=\left(\mathcal{G}_{00}-\hat{g}^{-1}-\tilde{v} F^{-1} v\right) \operatorname{det} F
\end{gathered}
$$

and

$$
F^{-1}=G^{-1}-\hat{f}^{-1}\left[G\left(G+\hat{f}^{-1}\right)\right]^{-1}
$$

into (3.24), and using (3.28) we obtain, for $P_{0}=E$.

$$
D(E, \vec{P})=\frac{i}{g} \frac{1+4 \hat{f}^{-1}(6-\hat{E})^{-1} \tilde{w} G\left(G+\hat{f}^{-1}\right)^{-1} w}{1-(6-\hat{E}) \hat{g}^{-1}+4 \hat{f}^{-1}(6-\hat{E})^{-1} \tilde{w} G\left(G+\hat{f}^{-1}\right)^{-1} w}
$$

This expression is especially useful in the case of a narrow width resonance boson, when $\hat{f}$ is large.

The pole position

$$
E=E_{\text {pole }}
$$

is given by

$$
D^{-1}\left(E_{\text {pole }}, \vec{P}\right)=0 \text {. }
$$

In a series expansion in powers of $\hat{f}^{-1}$, we find 


$$
\operatorname{Re} E_{\text {pole }}=12 t-g-24 t \hat{f}^{-1}+4 t \hat{f}^{-1} \sum_{\alpha=1}^{3}\left(1-\cos P_{\alpha} \ell\right)+O\left(\hat{f}^{-2}\right)
$$

and

$$
\operatorname{lm} E_{\text {pole }}=8 t \hat{f}^{-2} \operatorname{lm} \tilde{w} G^{-1} w+O\left(\hat{f}^{-3}\right)
$$

From (3.35), one sees that the hopping parameter for the boson is $2 t / \hat{f}$ for large $\hat{f}$, smaller than that of the fermion by a factor $2 / \hat{f}$.

When $\vec{P}=0$.

$$
w=\left(\begin{array}{l}
1 \\
1 \\
1
\end{array}\right), \quad v=\mathcal{G}_{10}\left(\begin{array}{l}
1 \\
1 \\
1
\end{array}\right)
$$

therefore, on account of (3.28), $w$ is an eigenvector of $G$ and

$$
\mathcal{G}_{10}(E, 0)=\left(1-\frac{\hat{E}}{6}\right) \mathcal{G}_{00}(E, 0)-\frac{1}{6}
$$

The boson propagator becomes

$$
D(E, 0)=\frac{i}{g} \frac{1+12 \hat{f}^{-1} \mathcal{G}_{10}\left[(6-\hat{E}) \mathcal{G}_{10}+2 \hat{f}^{-1}\right]^{-1}}{1-(6-\hat{E}) \hat{g}^{-1}+12 \hat{f}^{-1} \mathcal{G}_{10}\left[(6-\hat{E}) \mathcal{G}_{10}+2 \hat{f}^{-1}\right]^{-1}}
$$

and correspondingly (3.36) reduces to (neglecting $O\left(\hat{f}^{-3}\right)$ )

$$
\operatorname{Im} E_{\text {pole }}=-8 t \hat{f}^{-2}\left|\mathcal{G}_{01}(E, 0)\right|^{-2} \operatorname{Im} \mathcal{G}_{00}(E, 0)
$$

which is negative. From (3.39), one can verify that $D^{-1}\left(E_{\text {pole }}, 0\right)=0$ is the same as $E=E_{\text {pole }}$ satisfying (2.30), in accordance with the equivalence theorem.

\subsection{Two Fermion Scattering Amplitude}

The dressed vertex function $-i V$ for the transition (3.11) is given by the sum of all corresponding one-particle irreducible diagrams which, through the use of Figure $6(a)$, can be reduced to the sum of two diagrams given by Figure $7(a)$. The 
evaluation is straightforward. By using (3.14) and following steps essentially the same as (3.21)-(3.30), we find (when $\xi_{0} \rightarrow \infty$ )

$$
\begin{aligned}
<b\left|V\left(P_{0}, \vec{P}\right)\right| \vec{p}>=-\frac{4 t \hat{f}^{-1}}{\sqrt{\mathcal{N}}}\left[6-\hat{E}+4 \hat{f}^{-1} \tilde{w} G\left(G+\hat{f}^{-1}\right)^{-1} w\right]^{-1} \\
\cdot\left[-(6-\hat{E}-2 \tilde{w} \hat{u}) 2 \tilde{w} G\left(G+\hat{f}^{-1}\right)^{-1} w+(6-\hat{E}) \tilde{w}(G+\hat{f})^{-1} \hat{u}\right]
\end{aligned}
$$

where

$$
\hat{u}=\left(\begin{array}{c}
\cos p_{1} \ell \\
\cos p_{2} \ell \\
\cos p_{3} \ell
\end{array}\right)
$$

The two-fermion spin-singlet scattering amplitude for the process (3.8) is given by Figure $7(b)$ :

$$
\begin{aligned}
\left\langle\vec{q}\left|\Gamma\left(P_{0}, \vec{P}\right)\right| \vec{p}\right\rangle= & \left.<\vec{q}\left|\hat{\Gamma}\left(P_{0}, \vec{P}\right)\right| \vec{p}\right\rangle \\
& +<\vec{q}\left|V\left(P_{0}, \vec{P}\right)\right| b>D\left(P_{0}, \vec{P}\right)\left\langle b\left|V\left(P_{0}, \vec{P}\right)\right| \vec{p}\right\rangle,
\end{aligned}
$$

in which $\left\langle\vec{q}\left|V\left(P_{0}, \vec{P}\right)\right| b\right\rangle=\left\langle b\left|V\left(P_{0}, \vec{P}\right)\right| \vec{q}\right\rangle$. In the limit $\xi_{0} \rightarrow \infty$ the above expression reduces to the same $(\vec{q}|\gamma(E, \vec{P})| \vec{p})$ of $(2.15)$ on the mass-shell; the proof is given in Appendix $D$.

The scattering length $a$ is determined by the amplitude (3.43) at $P_{0}=0$ and $\vec{P}=\vec{p}=\vec{q}=0$. Setting

$$
<0|r(0,0)| 0>=\frac{2 t}{\mathcal{N}} \xi
$$

we have

$$
a=\frac{\ell}{4 \pi} \xi
$$

and

$$
\xi=\frac{\hat{g}\left(1+2 \hat{f}^{-1}-6 \hat{g}^{-1}\right)}{\hat{g}\left(g_{1}+2 g_{0} \hat{f}^{-1}\right)-2\left(3 g_{1}+\hat{f}^{-1}\right)}
$$

with $g_{0}=\mathcal{G}_{00}(0,0)$ given by $(2.38)$ and

$$
g_{1}=\mathcal{G}_{10}(0,0)=g_{0}-\frac{1}{6}
$$




\section{4. $Q=3$ AND 4 SECTORS OF $H$}

\subsection{Boson-Fermion Scattering}

From $H_{t}$ and $H_{\text {core }}$ of (3.5) and (1.31), the vertex for the scattering of a boson $b$ of momentum $\frac{\vec{p}}{2}+\vec{p}$ and a fermion of momentum $\frac{\vec{p}}{2}-\vec{p}$ and spin $\sigma=\uparrow$ or $\downarrow$.

$$
b\left(\frac{\vec{P}}{2}+\vec{p}\right)+f\left(\frac{\vec{P}}{2}-\vec{q}, \sigma\right) \rightarrow b\left(\frac{\vec{P}}{2}+\vec{q}\right)+f\left(\frac{\vec{P}}{2}-\vec{q}, \sigma\right)
$$

(given by Figure $8(a)$ ) is

$$
-i \frac{1}{\mathcal{N}} 2 t \tilde{U}(\vec{q}) \lambda^{-1} U(\vec{p})
$$

where $U(\vec{p})$ is a $7 \times 1$ column matrix, given by

$$
U(\vec{p})=\left(\begin{array}{c}
1 \\
\cos p_{1} \ell \\
\cos p_{2} \ell \\
\cos p_{3} \ell \\
\sin p_{1} \ell \\
\sin p_{2} \ell \\
\sin p_{3} \ell
\end{array}\right)
$$

and $\lambda$ is a $7 \times 7$ diagonal matrix whose diagonal elements are

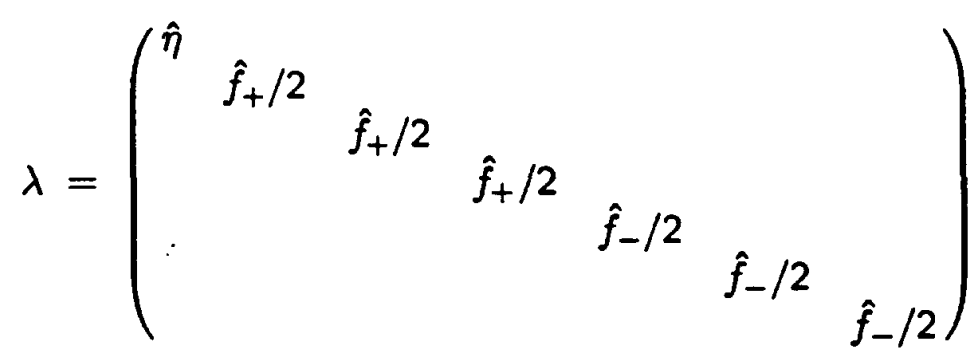

where

$$
\hat{\eta}=\eta_{0} / 2 t, \quad \hat{f}_{ \pm}=\hat{f} \pm 2
$$

and $\hat{f}=f / 2 t$, as before. Figure $8(\mathrm{c})$ gives the sum of diagrams for the scattering amplitude $\left\langle\vec{q}\left|S\left(P_{0}, \vec{P}\right)\right| \vec{p}\right\rangle$ times $-i$; in turn, $-i S$ can be decomposed into the chain sum $-i<\vec{q}\left|\hat{S}\left(P_{0}, \vec{P}\right)\right| \vec{p}>$ of Figure $8(\mathrm{~b})$, plus the remaining diagrams 
in Figure $8(c)$, all involving three-body scattering; the latter will be denoted by $\left.-i<\vec{q}\left|\delta S\left(P_{0}, \vec{P}\right)\right| \vec{p}\right\rangle$. It is straightforward to verify that

$$
<\vec{q}\left|\hat{S}\left(P_{0}, \vec{P}\right)\right| \vec{p}>=\frac{2 t}{\mathcal{N}} \tilde{U}(\vec{q}) \wedge\left(P_{0}, \vec{P}\right) U(\vec{p})
$$

where

$$
\Lambda\left(P_{0}, \vec{P}\right)^{-1}=\lambda^{-1}+i \sum_{\vec{k}} \frac{2 t}{\mathcal{N}} D\left(P_{0}-\epsilon_{\frac{p}{2}-\vec{k}}, \frac{\vec{P}}{2}+\vec{k}\right) U(\vec{k}) \tilde{U}(\vec{k})
$$

and

$$
\left.<\vec{q}\left|\delta S\left(P_{0}, \vec{P}\right)\right| \vec{p}\right\rangle=O\left(\hat{f}^{-2}\right)
$$

Hence, for $\vec{P}=\vec{p}=\vec{q}=0$ and $P_{0}=M \equiv 12 t-g-\left(48 t^{2} / f\right)+O\left(\hat{f}^{-2}\right)$, the on-mass-shell value (3.35), we have

$$
<0|S(M, 0)| 0>=\frac{2 t}{\mathcal{N}} \eta
$$

where, as $\eta_{0} \rightarrow \infty$,

$$
\eta=\frac{1}{2 g_{1}}\left[1+\hat{f}^{-1}\left(2-\frac{1}{3 g_{1}}\right)+O\left(\hat{f}^{-2}\right)\right]
$$

and

$$
g_{1}=g_{0}-\frac{1}{6}=0.08606
$$

\subsection{Boson-Boson Scattering}

The vertex for the scattering of two bosons,

$$
b\left(\frac{\vec{P}}{2}+\vec{p}\right)+b\left(\frac{\vec{P}}{2}-\vec{p}\right) \rightarrow b\left(\frac{\vec{P}}{2}+\vec{q}\right)+b\left(\frac{\vec{P}}{2}-\vec{q}\right)
$$

given by Figure $9(a)$, is

$$
-i \frac{2 t}{\mathcal{N}} \tilde{u}(\vec{q}) \kappa^{\prime} u(\vec{p})
$$

where $\kappa^{\prime}$ is a $4 \times 4$ diagonal matrix, whose diagonal matrix elements are, similar to $(3.10)$, 


$$
\kappa^{\prime}=\frac{1}{2 t}\left(\begin{array}{llll}
\zeta_{0} & & & \\
& f & & \\
& & f & \\
& & & f
\end{array}\right)
$$

Figure $9(\mathrm{c})$ gives the sum of diagrams for the scattering amplitude $\left\langle\vec{q}\left|T\left(P_{0}, \vec{P}\right)\right| \vec{p}\right\rangle$ times $-i$, which in turn can be decomposed into the chain sum $-i\left\langle\vec{q}\left|\hat{T}\left(P_{0}, \vec{P}\right)\right| \vec{p}\right\rangle$ (which satisfies the integral relation specified by Figure $9(b)$ ) plus the remaining diagrams in $9(c)$, all involving three-body scattering; the latter will be denoted by $\left.-i<\vec{q}\left|\delta T\left(P_{0}, \vec{P}\right)\right| \vec{p}\right\rangle$. One can readily verify that

$$
\left\langle\vec{q}\left|\hat{T}\left(P_{0}, \vec{P}\right)\right| \vec{p}\right\rangle=\frac{2 t}{\mathcal{N}} \tilde{u}(\vec{q}) \kappa^{\prime}\left(1+\frac{\hat{f}}{2} \mathcal{G} \kappa^{\prime}\right)^{-1} u(\vec{p})
$$

where $\mathcal{G}=\mathcal{G}\left(P_{0}, \vec{P}\right)$ is the $4 \times 4$ matrix of (2.13), and

$$
\left.<\vec{q}\left|\delta T\left(P_{0}, \vec{P}\right)\right| \vec{p}\right\rangle=O\left(\hat{f}^{-2}\right)
$$

Hence, for $\vec{P}=\vec{p}=\vec{q}=0, P_{0}$ is the zero kinetic energy on-mass-shell value

$$
P_{0}=2 M=2\left[12 t-g-\left(48 t^{2} / \hat{f}\right)+O\left(\hat{f}^{-2}\right)\right] ;
$$

the corresponding scattering length is determined by (as in (3.44)-(3.45))

$$
<0|T(2 M, 0)| 0>=\frac{2 t}{\mathcal{N}} \zeta
$$

where, as $\zeta_{0} \rightarrow \infty$.

$$
\zeta=\frac{2}{\hat{f} g_{1}}+O\left(\hat{f}^{-2}\right)
$$

with $g_{1}$ given by (4.11).

The many-body sectors of the same model will be analysed in a separate publication.

\section{ACKNOWLEDGMENT}

We wish to thank Y. Pang for helpful advice.

$$
J-1<\hat{f}_{-} / 2
$$

where 


\section{APPENDIX A}

In this appendix, we shall analyze the wave function of the scattering state of two fermions in the coordinate space and derive the expressions (2.28) and (2.29) for the phase shifts. The total momentum $\vec{P}$ of the two fermions is assumed to be zero throughout the discussion, so that the full cubic symmetry is realized.

\section{A.1. The Two-fermion Scattering Wave Function}

In the coordinate space, the two-fermion wave function with $\vec{P}=0$ is, up to a normalization factor, the Fourier transformation of (2.10); i.e.

$$
\psi(\vec{r} \mid \vec{p})=\sum_{\vec{q}} F_{0}(\vec{q} \mid \vec{p}) e^{i \vec{q} \vec{r}}=\hat{\psi}(\vec{r})+\psi_{\mathrm{sc}}(\vec{r}),
$$

where

$$
\hat{\psi}(\vec{r})=\cos \vec{p} \cdot \vec{r}
$$

and, as $\mathcal{N} \rightarrow \infty$.

$$
\psi_{\mathrm{sc}}(\vec{r})=\ell^{3} \int \frac{d^{3} \vec{q}}{(2 \pi)^{3}} \frac{(\vec{q}|\gamma(E, 0)| \vec{p})}{E-2 \epsilon_{\vec{p}}+i 0+} e^{i \vec{q} \vec{r}}
$$

with $\vec{r}$ the relative coordinate of the two fermions, $\vec{p}$ the initial relative momentum and $(\vec{q}|\gamma(E, 0)| \vec{p})$ given by (2.19). The wave function (A.1) is an eigenfunction of the Hamiltonian (1.22) with the energy $E=2 \epsilon_{\bar{p}}$.

The 24-member proper cubic group has five irreducible representations: There are two one-dimensional representations $A_{1}$ and $A_{2}$ (represented by the functions 1 and $x y z$ ), one two-dimensional representation $E$ (consisting of the doublet functions $x^{2}-y^{2}$ and $\left.x^{2}+y^{2}-2 z^{2}\right)$ and two three-dimensional representations, $T_{1}$ and $T_{2}$ (represented by two triplets $(x, y, z)$ and $(y z, z x, x y)$ ). Out of the four components of (2.12), two are type $A$

$$
s(\vec{p})=1
$$

and

$$
s^{\prime}(\vec{p})=\sum_{\alpha} \cos p_{\alpha} \ell
$$

the other two form a doublet of type $E$.

$$
d_{1}(\vec{p})=\frac{1}{\sqrt{2}}\left(\cos p_{1} \ell-\cos p_{2} \ell\right)
$$


and

$$
d_{2}(\vec{p})=\frac{1}{\sqrt{6}}\left(\cos p_{1} \ell+\cos p_{2} \ell-2 \cos p_{3} \ell\right) .
$$

If we expand (A.4)-(A.7) to the second order in the momentum $\vec{p}$ and examine the dependence of the expansion on the orientation $\hat{p}$, we find that (A.4) and (A.5) are isotropic while (A.6) and (A.7) are proportional to the components of the rank two spherical harmonics. Therefore we call (A.4) and (A.5) s-waves, and (A.6) and (A.7) $d$-waves. The $X$ and $Y$ in (2.21) and (2.22), written in terms of (A.4)-(A.5) read

$$
\begin{aligned}
X= & \frac{12}{\hat{f}}\left(-\hat{g}\left[2+\hat{f}(6-\hat{E}) \mathcal{G}_{01}(E)\right] s(\vec{q}) s(\vec{p})+2 \sqrt{3} \hat{g} \hat{f} \mathcal{G}_{01}(E)\right. \\
& {\left[s(\vec{q}) s^{\prime}(\vec{p})+s^{\prime}(\vec{q}) s(\vec{p})\right]+2 \hat{f}\left(1-\hat{g} \mathcal{G}_{00}(E)\right) s^{\prime}(\vec{q}) s^{\prime}(\vec{p}) }
\end{aligned}
$$

and

$$
Y=\hat{f}\left[1+\hat{f}\left(\mathcal{G}_{11}-\mathcal{G}_{12}\right)\right]^{-1}\left(d_{1}(\vec{q}) d_{1}(\vec{p})+\left(d_{2}(\vec{q}) d_{2}(\vec{p})\right)\right.
$$

For the scattering matrix, only the on-mass-shell elements are relevant; hence, $2 \epsilon_{\vec{q}}=$ $2 \epsilon_{\bar{p}}=E$ and, on account of (2.5), $s$ and $s^{\prime}$ are proportional to each other; therefore, in the large-distance asymptotic region, there is only one independent $s$-wave in (A.4)-(A.5), and the $X$ of (A.8) becomes

$$
X=\left(\hat{E}-e_{-}\right)\left(\hat{E}-e_{+}\right) s(\vec{q}) s(\vec{p})
$$

on the mass shell. The functions $s(\vec{p}), d_{1}(\vec{p})$ and $d_{2}(\vec{p})$ satisfy the following relations on the mass shell:

$$
\begin{gathered}
\ell^{3} \int \frac{d^{3} \vec{q}}{(2 \pi)^{3}} s^{2}(\vec{p}) \delta\left(2 \epsilon_{\vec{p}}-E\right)=\frac{1}{\pi} \operatorname{Im} \mathcal{G}_{00}(E, 0), \\
\ell^{3} \int \frac{d^{3} \vec{q}}{(2 \pi)^{3}} d_{m}^{2}(\dot{\vec{p}}) \delta\left(2 \epsilon_{\vec{p}}-E\right)=\frac{1}{\pi} \operatorname{lm}\left(\mathcal{G}_{11}(E, 0)-\mathcal{G}_{12}(E, 0)\right), \\
\ell^{3} \int \frac{d^{3} \vec{q}}{(2 \pi)^{3}} s(\vec{p}) d_{m}(\vec{p}) \delta\left(2 \epsilon_{\vec{p}}-E\right)=\ell^{3} \int \frac{d^{3} \vec{q}}{(2 \pi)^{3}} d_{1}(\vec{p}) d_{2}(\vec{p}) \delta\left(2 \epsilon_{\vec{p}}-E\right)=0
\end{gathered}
$$

with the subscript $m=1$ or 2 .

Analogous to the partial wave analysis of a scattering process in the continuum, we project out the $s$ - and $d$-partial waves from the wave functions (A.1) and (A.2): 


$$
\begin{gathered}
\psi_{s}(\vec{r})=\ell^{3} \int \frac{d^{3} \vec{p}}{(2 \pi)^{3}} \psi(\vec{r} \mid \vec{p}) \delta\left(2 \epsilon_{\vec{p}}-E\right), \\
\hat{\psi}_{s}(\vec{r})=\ell^{3} \int \frac{d^{3} \vec{p}}{(2 \pi)^{3}} \cos \vec{p} \cdot \vec{r} \delta\left(2 \epsilon_{\vec{p}}-E\right), \\
\psi_{d, m}(\vec{r})=\ell^{3} \int \frac{d^{3} \vec{p}}{(2 \pi)^{3}} d_{m}(\vec{p}) \psi(\vec{r} \mid \vec{p}) \delta\left(2 \epsilon_{\vec{p}}-E\right), \\
\hat{\psi}_{d, m}(\vec{r})=\ell^{3} \int \frac{d^{3} \vec{p}}{(2 \pi)^{3}} d_{m}(\vec{p}) \cos \vec{p} \cdot \vec{r} \delta\left(2 \epsilon_{\vec{p}}-E\right) .
\end{gathered}
$$

Of course, there are functions of $\vec{p}$ other than those listed in (A.4)-(A.7) which also transform according to the irreducible representations of the cubic group and are orthogonal to those of (A.4)-(A.7) on the mass shell. However, these functions do not enter into the scattering described by the Hamiltonian (1.22).

\section{A.2. Asymptotic Behavior and Phase Shifts}

The Brillouin zone for a simple cubic lattice is bounded in the momentum space by the condition $-\pi \leq p_{\alpha} \ell \leq \pi$ with $\alpha=1,2$ and 3 . Denote the surface of constant energy, $2 \epsilon_{\bar{p}}=E$, within the Brillouin zone by $\mathcal{S}_{E}$. With the $\epsilon_{\bar{p}}$ given by (2.5), the surface $\mathcal{S}_{E}$ is closed within the zone for $0<E<8 t$ and connects continuously to its periodic images across the boundary of the zone for $E>8 t$. The group velocity of the fermion at $\vec{p}$ on $\mathcal{S}_{E}$ is

$$
\vec{v}_{\vec{p}}=\vec{\nabla}_{\vec{p}} \epsilon_{\vec{p}}
$$

Geometrically, $\vec{v}_{\vec{p}}$ is parallel to the normal of $\mathcal{S}_{E}$ at $\vec{p}$ and points from the side with $2 \epsilon_{\vec{p}}<E$ to the side with $2 \epsilon_{\vec{p}}>E$, and

$$
\vec{p} \cdot \vec{v}_{\vec{p}}>0
$$

for all $\vec{p}$ on $\mathcal{S}_{E}$.

Consider the scattering term (A.3) of the wave function (A.1). At large $r$. the contribution to the integral is dominated by the pole of the integrand, which corresponds to $\vec{q}$ on $\mathcal{S}_{E}$. Decompose the integration over $\vec{q}$ into an integration over the radial component $q$ and that over the solid angle spanned by $\hat{q}=\vec{q} / q$. By carrying out the radial integration to the leading order in $r^{-1}$, we find 


$$
\psi_{s c}(\vec{r})=-\frac{i}{2} \ell^{3} \int \frac{d^{2} \hat{q}}{(2 \pi)^{2}} \frac{q^{2}(\hat{q})}{\hat{q} \cdot \vec{v}_{\vec{q}}} e^{i \vec{q} \cdot \vec{r}} \theta\left(\hat{q} \cdot \vec{v}_{\vec{q}}\right)(\vec{q}|\gamma(E, 0)| \vec{p})
$$

where $\vec{q}$ is confined to $\mathcal{S}_{E}$ and the step function $\theta(\xi)$ is 0 for $\xi<0$ and 1 for $\xi>0$. The integration over the solid angle can be evaluated by the stationary phase approximation for large $r$. There are two points $\pm \vec{k}$ on $\mathcal{S}_{E}$ at which the phase of the integrand of (A.20) is stationary; the group velocity at $\vec{k}(-\vec{k})$ is parallel (antiparallel) to the vector $\vec{r}$. The antiparallel one is ruled out by the step function in (A.20) on account of (A.19). Physically, these two points give rise to the outgoing -wave and the incoming wave; here, the choice of the infinitesimal imaginary part in the denominator of (A.3) gives rise to the $\theta$-function in (A.20) and selects the outgoing wave. Set a Cartesian coordinate system $(\xi, \eta, \zeta)$ with its origin $\vec{q}=\vec{k}$, the $\zeta$-axis parallel to $\vec{r}$, and the $\xi \eta$-plane tangent to $\mathcal{S}_{E}$; the surface $\mathcal{S}_{E}$ can be parameterized in the neighborhood of $\vec{k}$ as

$$
\zeta=-\frac{1}{2}\left(\frac{\xi^{2}}{\rho_{1}}+\frac{\eta^{2}}{\rho_{2}}\right)
$$

with $\rho_{1}$ and $\rho_{2}$ the two principal radii of curvature of $\mathcal{S}_{E}$ at $\vec{q}=\vec{k}$. The phase of the exponential in (A.20) can be written as

$$
\vec{k} \cdot \vec{r}-\frac{r}{2}\left(\frac{\xi^{2}}{\rho_{1}}+\frac{\eta^{2}}{\rho_{2}}\right)
$$

Replacing $q^{2}(\hat{q}) d^{2} \hat{q}$ in (A.19) by $d \xi d \eta$, we find the integration to be Gaussian and derive

$$
\psi(\vec{r} \mid \vec{p})=\cos \vec{p} \cdot \vec{r}-\frac{\ell^{3}}{4 \pi} \frac{\sqrt{\left|\rho_{1} \rho_{2}\right|}}{\hat{k} \cdot \vec{v}_{\vec{k}}}(\vec{k}|\gamma(E, 0)| \vec{p}) \frac{e^{i(\vec{k} \cdot \vec{r}+\chi)}}{r}
$$

where $\chi=0$ for $\rho_{1}, \rho_{2}$ both $>0, \chi=\pi$ for $\rho_{1}, \rho_{2}$ both $<0$ and $\chi=\frac{\pi}{2}$, otherwise. The approximation breaks down if one or two of $\rho_{1}$ and $\rho_{2}$ vanish.

We evaluate next the large $r$ behavior of the $s$-partial wave (A.15) and the $d$-partial wave (A.17) of the plane wave (A.2). At a fixed $\vec{r}$, we average (A.2) over all $\vec{p}$ on the surface $\mathcal{S}_{E}$, defined by $E=2 \epsilon_{\vec{p}}$. The stationary phase approximation again picks out the same two points $\vec{p}=\vec{k}$ and $-\vec{k}$ on $\mathcal{S}_{E}$; however, now both 
outgoing and incoming waves contribute. Neglecting $O\left(r^{-2}\right)$, the result for the $s$-wave is

$$
\hat{\psi}_{s}(\vec{r})=\frac{i \ell^{3}}{4 \pi} \frac{\sqrt{\left|\rho_{1} \rho_{2}\right|}}{\hat{k} \cdot \vec{v}_{\vec{k}}} \frac{\sin (\vec{k} \cdot \vec{r}+\chi)}{r}
$$

and that for the $d$-wave is

$$
\hat{\psi}_{d, m}(\vec{r})=\frac{i \ell^{3}}{4 \pi} \frac{\sqrt{\left|\rho_{1} \rho_{2}\right|}}{\hat{k} \cdot \vec{v}_{\vec{k}}} \frac{\sin (\vec{k} \cdot \vec{r}+\chi)}{r} d_{m}(\vec{k})
$$

with $m=1$ or 2 , as before.

Combining (A.23)-(A.25) and utilizing (A.9)-(A.12), we obtain the large-distance asymptotic behavior of the $s$ - and $d$-partial waves (A.14) and (A.16) of the wave function (A.1):

$$
\psi_{s}(\vec{r})=e^{i \delta_{s}(E)} \frac{i \ell^{3}}{4 \pi} \frac{\sqrt{\left|\rho_{1} \rho_{2}\right|}}{\hat{k} \cdot \vec{v}_{\vec{k}}} \frac{\sin \left(\vec{k} \cdot \vec{r}+\chi+\delta_{s}(E)\right)}{r}
$$

and

$$
\psi_{d, m}(\vec{r})=e^{i \delta_{d}(E)} \frac{i \ell^{3}}{4 \pi} \frac{\sqrt{\left|\rho_{1} \rho_{2}\right|}}{\hat{k} \cdot \vec{v}_{\vec{k}}} \frac{\sin \left(\vec{k} \cdot \vec{r}+\chi+\delta_{d}(E)\right)}{r} d_{m}(\vec{k})
$$

with the phase shifts given by (2.28) and (2.29). 


\section{APPENDIX B}

\section{Analyticity on the Physical Sheet}

In this appendix, we prove that the scattering amplitude $\langle\vec{q}|\gamma(E, 0)| \vec{p}\rangle$ of (2.19) is analytic on the physical complex $E$-plane, excluding the real axis. Consider first a large but finite lattice of $\mathcal{N}$ sites. As we shall see, the solutions of (2.23) = 0.

$$
Z=\left(\hat{E}-e_{0}\right)+\left(\hat{E}-e_{+}\right)\left(\hat{E}-e_{-}\right) \mathcal{G}_{\infty}=0
$$

must all be real. For $\mathcal{N}$ finite and $\hat{E}=E / 2 t$ complex, we can omit the $i 0+$ in (2.13); since $\vec{P}=0$.

$$
\mathcal{G}_{00}=\frac{2 t}{\overline{\mathcal{N}}} \sum_{\vec{q}} \frac{1}{2 \epsilon_{\bar{q}}-E}
$$

which, with $\hat{\epsilon}_{\vec{q}}=\epsilon_{\vec{q}} / 2 t$, can be expressed in the form

$$
\mathcal{G}_{00}(\hat{E})=\prod_{\vec{q}}\left(2 \hat{\epsilon}_{\vec{q}}-\hat{E}\right)^{-1} \prod_{n=1}^{\mathcal{N}-1}\left(z_{n}-\hat{E}\right)
$$

where $z_{n}$ are the zeroes of $\mathcal{G}_{00}(\hat{E})$. Noting that the coefficient of $\hat{E}^{\mathcal{N}+1}$ is zero in the product $Z \prod_{\vec{q}}\left(2 \hat{\epsilon}_{\vec{q}}-\hat{E}\right)$, we can write $Z=0$ as

$$
\prod_{n=1}^{\mathcal{N}}\left(\hat{E}-\hat{E}_{n}\right)=0
$$

At the roots, $\hat{E}=\hat{E}_{n}$, we have

$$
\mathcal{G}_{\infty}=I^{-1}(\hat{E}) \equiv \frac{e_{0}-\hat{E}}{\left(e_{+}-\hat{E}\right)\left(e_{-}-\hat{E}\right)}
$$

with $I(\hat{E})$ the same one given by $(2.31)$.

Recall that when $\hat{E} \rightarrow \pm \infty$

$$
\mathcal{G}_{00}(\hat{E}) \rightarrow-\hat{E}^{-1}-6 \hat{E}^{-2}+O\left(\hat{E}^{-3}\right)
$$




$$
I^{-1}(\hat{E}) \rightarrow-\hat{E}^{-1}-\left(6+\frac{12}{\hat{f}}\right) \hat{E}^{-2}+O\left(\hat{E}^{-3}\right)
$$

and therefore

$$
\mathcal{G}_{00}(\hat{E})-I^{-1}(\hat{E}) \rightarrow\left(\frac{12}{\hat{f}}\right) \hat{E}^{-2}+O\left(\hat{E}^{-3}\right) .
$$

For $\hat{E}$ real, (2.34) gives $\frac{\partial}{\partial \hat{E}}\left(I-\mathcal{G}_{00}^{-1}\right)>0$; i.e.

$$
\frac{\partial \mathcal{G}_{00}}{\partial \hat{E}}>\left(I \mathcal{G}_{00}\right)^{2} \frac{\partial I^{-1}}{\partial \hat{E}} .
$$

Hence, when the root $\hat{E}_{n}$ of (B.4) is real, at $\hat{E}=\hat{E}_{n}$ we have $I \mathcal{G}_{00}=1$ and

$$
\frac{\partial}{\partial \hat{E}}\left(\mathcal{G}_{00}-I^{-1}\right)>0 .
$$

In addition, from (2.24)-(2.27), $e_{0} \leq e_{-}$and $e_{+}$is always positive (since $\hat{g}$ and $\hat{f}$ are positive). Therefore,

$$
I^{-1}(\hat{E})>0 \quad \text { for } \quad e_{+}>\hat{E}>e_{-} .
$$

To simplify the discussion, we assume the surface of the lattice to be sufficiently irregular so that there is no degeneracy in $\epsilon_{\bar{q}}$; yet these irregularities are minute and confined to a shallow layer parallel to the surface, so that in the limit $\mathcal{N} \rightarrow \infty$, $\vec{q}$ becomes the usual continuous momentum bounded by the Brillouin zone, $\hat{\epsilon}_{\bar{q}}=$ $\sum_{\alpha}\left(1-\cos q_{\alpha} \ell\right) \leq 6$. For $\mathcal{N}$ finite, write $2 \hat{\epsilon}_{\vec{q}}=e_{\vec{q}}$ and order the set $\left\{2 \hat{\epsilon}_{\vec{q}}\right\}$ as

$$
e_{1}<e_{2}<\cdot<e_{\mathcal{N}}
$$

which are all positive: Equation (B.2) becomes

$$
\mathcal{G}_{00}=\sum_{n=1}^{\mathcal{N}} \frac{1}{e_{n}-\hat{E}}
$$

In Figure 10, the solid line represents $\mathcal{G}_{00}$ vs. $\hat{E}$ and the dashed line $I^{-1}$ vs. $\hat{E}$. There are altogether $\mathcal{N}+1$ segments of the solid line. As $\hat{E}$ increases from $-\infty . \mathcal{G}_{00}$ varies from $0+$ to $+\infty$ at $\hat{E}=e_{1}-$, then from $-\infty$ at $\hat{E}=e_{1}+$ to $+\infty$ at $\hat{E}=e_{2}-$, etc. There are three segments of the dashed line. As $\hat{E}$ 
increases from $-\infty, I^{-1}$ varies from $0+$ to $-\infty$ at $\hat{E}=e_{-}$, then from $+\infty$ at $\hat{E}=e_{-}+$decreases to a positive minimum between $e_{-}$and $e_{+}$(in accordance with (B.10)) and increases to $+\infty$ at $\hat{E}=e_{+}-$; it then jumps to $-\infty$ at $\hat{E}=e_{-}+$ and increases to $0-$ at $\hat{E}=+\infty$. Because of (B.9), each dashed-line segment can only intersect a solid-line segment at most once. Furthermore, on account of (B.7),

$$
\mathcal{G}_{00}>I^{-1} \text { as } \hat{E} \rightarrow \pm \infty
$$

We distinguish the following two cases (illustrated by Figures $10(a)$ and $10(b)$ ):

(a) $e_{-}<e_{1}$ and $e_{n}<e_{+}<e_{n+1}$.

There are $n$ intersections between the solid- and dashed-line segments from $\hat{E}=-\infty$ to $e_{n}$, and $\mathcal{N}-n$ intersections between those from $\hat{E}=e_{n+1}$ to $+\infty$. with the solid-line segment between $e_{n}$ and $e_{n+1}$ not intersected (since there are at most $\mathcal{N}$ intersections).

(b) $e_{m}<e_{-}<e_{m+1}$ and $e_{n}<e_{+}<e_{n+1}$.

There is no intersection from $\hat{E}=-\infty$ to $e_{1}, m-1$ intersections from $\hat{E}=$ $e_{1}$ to $e_{m}, n-m-1$ intersections from $e_{m+1}$ to $e_{n}$ and $\mathcal{N}-n$ intersections from $e_{n+1}$ to $+\infty$. The solid-line segment between $e_{m}$ and $e_{m+1}$ has two intersections with two different dashed-line segments, and the one between $e_{n}$ and $e_{n+1}$ not intersected (since there are at most $\mathcal{N}$ intersections).

The same considerations can also be extended to the physically extreme cases $e_{+}>e_{\mathcal{N}}$ or/and $e_{+}>e_{-}>e_{\mathcal{N}}$. In all of these situations one sees that there are $\mathcal{N}$ real roots $\hat{E}_{1}, \cdot \cdot, \hat{E}_{\mathcal{N}}$. The analyticity on the physical $E$-plane follows; it remains valid in the limit $\mathcal{N} \rightarrow \infty$. 


\section{APPENDIX C}

Exact Properties of Scattering Amplitude Poles on the "Second Sheet"

Poles of the scattering amplitude are determined by the equation

$$
Z(\hat{E})=0
$$

where $Z$ is given by (2.23) and therefore shares the analytic structure of $\mathcal{G}_{00}(\hat{E})$. (As in Sections 2.2-2.3, the total momentum $\vec{P}$ is zero and $\hat{E}=E / 2 t$. Instead of (2.48)-(2.49), we retain the full cubic symmetry of the problem.) On the physical sheet $\mathcal{G}_{00}$ is given by (2.13) as (for an infinite cubic lattice in $N$ dimensions)

$$
\mathcal{G}_{00}(\hat{E})=\frac{1}{(2 \pi)^{N}} \int_{-\pi}^{\pi} d q_{1} \cdots d q_{N} \frac{1}{\sum_{\alpha=1}^{N}\left(2-2 \cos q_{\alpha}\right)-\hat{E}}
$$

which is manifestly analytic except for $0<\hat{E}<4 N$ on the real axis. For $\operatorname{Re} \hat{E}<0$ we may use the representation

$$
\frac{1}{M}=\int_{0}^{\infty} e^{-\lambda M} d \lambda(\operatorname{Re} M>0)
$$

to write (C.2) as

$$
\mathcal{G}_{00}(\hat{E})=\int_{0}^{\infty} e^{(\hat{E}-2 N) \lambda} I_{0}(2 \lambda)^{N} d \lambda
$$

where $I_{0}$ is the modified Bessel function,

$$
I_{0}(u)=\frac{1}{2 \pi} \int_{-\pi}^{\pi} e^{u \cos q} d q
$$

(This function has nothing to do with the $I_{0}$ defined in (2.41).)

We may continue (C.4) to positive real $\hat{E}$ with the aid of the functions

$$
L_{n}^{ \pm}(x) \equiv \int_{0}^{\infty} e^{-(x-2 N+4 n) \lambda} K_{0}(2 \lambda)^{N-n} K_{ \pm}(2 \lambda)^{n} d \lambda
$$

where $K_{0}$ is the modified Hankel function,

$$
K_{0}(u)=\int_{0}^{\infty} e^{-u \cosh \alpha} d \alpha \quad(\operatorname{Re} u>0)
$$


and $K_{ \pm}$is its continuation

$$
K_{ \pm}(u)=K_{0}\left(u e^{ \pm i \pi}\right)
$$

On account of the identity

$$
i \pi I_{0}(u)=K_{0}(u)-K_{+}(u)=K_{-}(u)-K_{0}(u)
$$

we have (still for $\hat{E}<0$ )

$$
\mathcal{G}_{00}(\hat{E})=\left(\frac{ \pm 1}{i \pi}\right)^{N} \sum_{n=0}^{N}\left(\begin{array}{l}
N \\
n
\end{array}\right)(-1)^{n} L_{n}^{ \pm}(4(N-n)-\hat{E})
$$

For simplicity, we shall now specialize to three dimensions, but the methods are evidently applicable for any $N$. For $N=3$ :

$$
\begin{aligned}
L_{0}^{+}(x)=L_{0}^{-}(x)=L_{0}(x) & =\int_{0}^{\infty} e^{-(x-6) \lambda} K_{0}^{3} d \lambda \\
L_{1}^{ \pm}(x) & =\int_{0}^{\infty} e^{-(x-2) \lambda} K_{0}^{2} K_{ \pm} d \lambda \\
L_{2}^{ \pm}(x) & =\int_{0}^{\infty} e^{-(x+2) \lambda} K_{0} K_{ \pm}^{2} d \lambda \\
L_{3}^{ \pm}(x) & =\int_{0}^{\infty} e^{-(x+6) \lambda} K_{ \pm}^{3} d \lambda
\end{aligned}
$$

where the argument $(2 \lambda)$ is everywhere understood, and

$$
\begin{aligned}
\mathcal{G}_{\infty}(\hat{E}) & =\frac{1}{(i \pi)^{3}}\left[L_{0}(12-\hat{E})-3 L_{1}^{+}(8-\hat{E})+3 L_{2}^{+}(4-\hat{E})-L_{3}^{+}(-\hat{E})\right] \\
& =\frac{1}{(-i \pi)^{3}}\left[L_{0}(12-\hat{E})-3 L_{1}^{-}(8-\hat{E})+3 L_{2}^{-}(4-\hat{E})-L_{3}^{-}(-\hat{E})\right]
\end{aligned}
$$

for $\operatorname{Re} \hat{E}<0$.

For $u \rightarrow+\infty$ we have $K_{0}(u) \propto \frac{e^{-u}}{\sqrt{u}}, K_{ \pm}(u) \propto \frac{e^{+u}}{\sqrt{u}}$ and therefore each $L_{n}^{ \pm}$is defined by $(C .11)$ for $\operatorname{Re} x>0$, but has a branch point at $x=0$. We may continue $L_{n}^{+}$counterclockwise about the origin by simultaneously deforming the $\lambda$-contour clockwise in (C.11) so that after rotating $180^{\circ} \lambda$ runs from 0 to 
$e^{-i \pi} \infty$; then replacing the symbol $\lambda$ by $\lambda^{\prime}=e^{-i \pi} \lambda$ where the new $\lambda$ runs from 0 to $\infty$, we have

$$
\begin{aligned}
L_{0}\left(x e^{i \pi}\right) & =\int_{0}^{\infty} e^{-\left(x e^{i \pi}-6\right) \lambda e^{-i \pi}} K_{0}\left(2 \lambda e^{-i \pi}\right)^{3} d \lambda e^{i \pi} \\
& =-\int_{0}^{\infty} e^{-(x+6) \lambda} K_{-}(2 \lambda)^{3} d \lambda \\
& =-L_{3}^{-}(x)
\end{aligned}
$$

and likewise

$$
\begin{aligned}
& L_{1}^{+}\left(x e^{i \pi}\right)=-L_{2}^{-}(x), \\
& L_{2}^{+}\left(x e^{i \pi}\right)=-L_{1}^{-}(x), \\
& L_{3}^{+}\left(x e^{i \pi}\right)=-L_{0}^{-}(x)
\end{aligned}
$$

where $x$ is taken on the sheet where (C.11) holds.

But $L_{n}^{+}$cannot be continued clockwise by these formulas, as the function $K_{0}\left(2 \lambda e^{2 i \pi}\right)$ does not have the needed exponential decay. However, we have

$$
\begin{aligned}
& L_{0}\left(x e^{-i \pi}\right)=-L_{3}^{+}(x), \\
& L_{1}^{-}\left(x e^{-i \pi}\right)=-L_{2}^{+}(x), \\
& L_{2}^{-}\left(x e^{-i \pi}\right)=-L_{1}^{+}(x), \\
& L_{3}^{-}\left(x e^{-i \pi}\right)=-L_{0}(x) .
\end{aligned}
$$

On account of the relation

$$
K_{+}+K_{-}=2 K_{0}
$$

which follows from (C.9), all the $L_{n}^{+}$can be expressed in terms of $L_{n^{\prime} \leq n}^{-}$and vice versa:

$$
\begin{aligned}
& L_{1}^{+}(x)=2 L_{0}(x+4)-L_{1}^{-}(x) \\
& L_{2}^{+}(x)=4 L_{0}(x+8)-4 L_{1}^{-}(x+4)+L_{2}^{-}(x) \\
& L_{3}^{+}(x)=8 L_{0}(x+12)-12 L_{1}^{-}(x+8)+6 L_{2}^{-}(x+4)-L_{3}^{-}(x)
\end{aligned}
$$

in which all terms are well-defined by (C.11) for positive $\operatorname{Re} x$.

Thus (C.13)-(C.15) suffice to continue all the $L$-functions to the whole domain $(-\pi<\arg x<\pi)$. Writing $G(\hat{E})$ for $\mathcal{G}_{00}(\hat{E})$ on the physical sheet, we have for Im $\hat{E}>0$, using the second line of (C.12), 


$$
\begin{aligned}
& 0<\operatorname{Re} \hat{E}<4: G(\hat{E})=\frac{1}{(-i \pi)^{3}}\left[L_{0}(12-\hat{E})-3 L_{1}^{-}(8-\hat{E})+3 L_{2}^{-}(4-\hat{E})+L_{0}(\hat{E})\right] \\
& \begin{aligned}
& 4<\operatorname{Re} \hat{E}<8: G(\hat{E})=\frac{1}{(-i \pi)^{3}}\left[L_{0}(12-\hat{E})-3 L_{1}^{-}(8-\hat{E})-3 L_{1}^{+}(\hat{E}-4)+L_{0}(\hat{E})\right] \\
& 8<\operatorname{Re} \hat{E}<12: G(\hat{E})=\frac{1}{(-i \pi)^{3}}\left[L_{0}(12-\hat{E})+3 L_{2}^{+}(\hat{E}-8)-3 L_{1}^{+}(\hat{E}-4)+L_{0}(\hat{E})\right] \\
& \operatorname{Re} \hat{E}>12: G(\hat{E})=\frac{1}{(-i \pi)^{3}}\left[-L_{3}^{+}(\hat{E}-12)+3 L_{2}^{+}(\hat{E}-8)-3 L_{1}^{+}(\hat{E}-4)+L_{0}(\hat{E})\right] \\
&=-G(12-\hat{E}) .
\end{aligned}
\end{aligned}
$$

For $\operatorname{lm} \hat{E}<0$ we have similar expressions:

$$
\begin{aligned}
& 0<\operatorname{Re} \hat{E}<4: G(\hat{E})=\frac{1}{(i \pi)^{3}}\left[L_{0}(12-\hat{E})-3 L_{1}^{+}(8-\hat{E})+3 L_{2}^{+}(4-\hat{E})+L_{0}(\hat{E})\right] \\
& 4<\operatorname{Re} \hat{E}<8: G(\hat{E})=\frac{1}{(i \pi)^{3}}\left[L_{0}(12-\hat{E})-3 L_{1}^{+}(8-\hat{E})-3 L_{1}^{-}(\hat{E}-4)+L_{0}(\hat{E})\right] \text {, } \\
& 8<\operatorname{Re} \hat{E}<12: G(\hat{E})=\frac{1}{(i \pi)^{3}}\left[L_{0}(12-\hat{E})+3 L_{2}^{-}(\hat{E}-8)-3 L_{1}^{-}(\hat{E}-4)+L_{0}(\hat{E})\right] \text {, } \\
& \operatorname{Re} \hat{E}>12: G(\hat{E})=\frac{1}{(i \pi)^{3}}\left[-L_{3}^{-}(\hat{E}-12)+3 L_{2}^{-}(\hat{E}-8)-3 L_{1}^{-}(\hat{E}-4)+L_{0}(\hat{E})\right] \\
& =-G(12-\hat{E}) \text {. }
\end{aligned}
$$

The agreement of the last lines, $G(\hat{E})=-G(12-\hat{E})$, shows that the cut on the real line does not extend to $E>12$. (This is no longer so on other sheets.)

Each of the four lines of (C.18) can be extended without formal modification to $\operatorname{Im} \hat{E}<0$, but the result (when re-expressed according to (C.17)) will not agree with (C.19) except in the last line, $\operatorname{Re} \hat{E}>12$. Thus one encounters singularities only at $E=0,4,8,12$; by going between these singularities, one arrives at any of three unphysical sheets. Still more sheets can be explored by following a nontrivial path encircling the various singularities in arbitrary sequence.

If any line of (C.18) is extended downward to $\operatorname{Im} \hat{E}<0$ and the $L^{+}$functions are replaced by $L^{-}$functions according to (C.17), we can continue to $\operatorname{Re} \hat{E}<0$ on the new sheet by a second application of (C.15), and then apply (C.17) or its inverse once more so as to express the result entirely in terms of $L^{+}$'s or of $L^{-1}$ s. These two expressions can serve as new starting points for continuation by (C.14) or (C.15) to a more remote sheet. Hence on every sheet $\mathcal{G}_{00}(\hat{E})$ is a linear combination 
of the same four functions which for $\operatorname{Re} \hat{E}<0$ are given according to (C.11) by $L_{0}(12-\hat{E}), L_{1}^{+}(8-\hat{E}), L_{2}^{+}(4-\hat{E}), L_{3}^{+}(-\hat{E})$; or, alternatively, of the four functions $L_{0}(12-\hat{E}), L_{1}^{-}(8-\hat{E}), L_{2}^{-}(4-\hat{E}), L_{3}^{-}(-\hat{E})$. Only the constant coefficents vary from sheet to sheet.

It follows that no singularities ever appear in $\mathcal{G}_{00}$ other than at $0,4,8,12$ (which are related to the Van Hove singularities ${ }^{7}$ ); but at $\infty$, except on the physical sheet, there may be a singularity of order $(\ln \hat{E})^{2} / \hat{E} ;$ in $N$ dimensions this becomes $(\ln \hat{E})^{N-1} / \hat{E}$. This induces a cut from 12 to $+\infty$ on the unphysical sheets.

We are particularly interested in the sheet reached by encircling the point $\hat{E}=$ 0 clockwise from the physical sheet. We shall call this the second sheet. The value of $\mathcal{G}_{00}(\hat{E})$ on the second sheet will be denoted by $\tilde{G}(\hat{E})$.

The process of alternately applying (C.15) and (C.17) is aided by defining new functions

$$
\begin{aligned}
& F_{0}(x)=\int_{0}^{\infty} e^{-(x+6) \lambda} I_{0}(2 \lambda)^{3} d \lambda=G(-x), \\
& F_{1}(x)=\int_{0}^{\infty} e^{-(x+2) \lambda} I_{0}(2 \lambda)^{2} K_{0}(2 \lambda) d \lambda, \\
& F_{2}(x)=\int_{0}^{\infty} e^{-(x-2) \lambda} I_{0}(2 \lambda) K_{0}(2 \lambda)^{2} d \lambda, \\
& F_{3}(x)=\int_{0}^{\infty} e^{-(x-6) \lambda} K_{0}(2 \lambda)^{3} d \lambda,
\end{aligned}
$$

all analytic for $\operatorname{Re} x>0$ and singular at $x=0$. Using (C.9), we have

$$
\begin{aligned}
& L_{0}(x)=F_{3}(x), \\
& L_{1}^{ \pm}(x)=F_{3}(x+4) \mp i \pi F_{2}(x), \\
& L_{2}^{ \pm}(x)=F_{3}(x+8) \mp 2 i \pi F_{2}(x+4)-\pi^{2} F_{1}(x), \\
& L_{3}^{ \pm}(x)=F_{3}(x+12) \mp 3 i \pi F_{2}(x+8)-3 \pi^{2} F_{1}(x+4) \pm i \pi^{3} F_{0}(x) .
\end{aligned}
$$

Now in the second line of (C.12), the only term singular at $\hat{E}=0$ is the last term, which is easily continued a full circle clockwise about the singularity: $L_{3}^{-}\left(x e^{-2 i \pi}\right)=-L_{0}\left(x e^{-i \pi}\right)=L_{3}^{+}(x)$. Hence, for $\hat{E}<0$.

$$
\begin{aligned}
\tilde{G}(\hat{E})-G(\hat{E}) & =\frac{1}{(-i \pi)^{3}}\left(L_{3}^{-}(-\hat{E})-L_{3}^{+}(-\hat{E})\right) \\
& =\frac{2}{(-i \pi)^{3}}\left(+3 i \pi F_{2}(8-\hat{E})-i \pi^{3} F_{0}(-\hat{E})\right) \\
& =\frac{6}{\pi^{2}} F_{2}(8-\hat{E})-2 F_{0}(-\hat{E})
\end{aligned}
$$


by substitution from (C.21); and since $F_{0}(-\hat{E})=G(\hat{E})$ by $(C .20)$, we have

$$
\tilde{G}(\hat{E})=\frac{6}{\pi^{2}} F_{2}(8-\hat{E})-G(\hat{E}) \text {. }
$$

Since $F_{2}(8-\hat{E})$ is analytic at $\hat{E}=0$, a second clockwise encirclement of the origin yieids $\tilde{\tilde{G}}(\hat{E})=\frac{6}{\pi^{2}} F_{2}(8-\hat{E})-\tilde{G}(\hat{E})=G(\hat{E})$. Thus the second sheet is the same as would have been reached by encircling the origin counterclockwise from the physical sheet.

This result generalizes to all four singularities; each one is locally of the "square root" type, so that encircling it twice in succession leads back to the same sheet. This follows from the fact that each $L$-function has a square root singularity at the origin. For example we have from (C.14) and (C.17), for $0<x<4$,

$$
\begin{aligned}
L_{2}^{+}\left(x e^{2 i \pi}\right) & =-L_{1}^{-}\left(x e^{i \pi}\right) \\
& =-2 L_{0}\left(x e^{i \pi}+4\right)+L_{1}^{+}\left(x e^{i \pi}\right) \\
& =-2 L_{0}(4-x)-L_{2}^{-}(x) \\
& =-2 L_{0}(4-x)-\left(4 L_{0}(x+8)-4 L_{1}^{+}(x+4)+L_{2}^{+}(x)\right) \\
& =M(x)-L_{2}^{+}(x)
\end{aligned}
$$

where $M(x)=4 L_{1}^{+}(x+4)-4 L_{0}(x+8)-2 L_{0}(4-x)$ is analytic at $x=0$. It follows that

$$
L_{2}^{+}\left(x e^{4 i \pi}\right)=M\left(x e^{2 i \pi}\right)-L_{2}^{+}\left(x e^{2 i \pi}\right)=M(x)-L_{2}^{+}\left(x e^{2 i \pi}\right)=L_{2}^{+}(x) .
$$

This works for all the $L$ 's, and hence for $\mathcal{G}_{00}$ at each singularity.

The square-root nature of each singularity holds in all odd dimensions, but in even dimensions the $L$-term in the last line of (C.24) becomes positive, and hence each singularity is logarithmic.

It is still possible to reach many distinct sheets by encircling different singularities successively. The fundamental homotopy group is not commutative.

We now develop. some facts about $\tilde{G}(e-i 0)$ for real $e$; that is, about $\mathcal{G}_{00}(\hat{E})$ on the second sheet just below the cut.

For $e<0, \tilde{G}(e-i 0)=\tilde{G}(e)$ is real by (C.23).

For $0<e<8, F_{2}(8-e)$ is still real and analytic by (C.20), and so $\operatorname{Im} \tilde{G}(e-$ $i 0)=-\operatorname{lm} G(e-i 0)>0$ by (C.2). 
To continue $F_{2}(8-\hat{E})$ counterclockwise about $\hat{E}=8$, we write the third line of (C.20) as

$$
F_{2}(x)=\int_{0}^{\infty} e^{-(x-2)} K_{0}^{2} \frac{1}{i \pi}\left(K_{0}-K_{+}\right) d \lambda=\frac{1}{i \pi}\left(L_{0}(x+4)-L_{1}^{+}(x)\right)
$$

so that, by (C.14),

$$
F_{2}\left(x e^{i \pi}\right)=\frac{1}{i \pi}\left(L_{0}(-x+4)-L_{2}^{-}(x)\right) \text { for } 0<x<4
$$

Substituting this along with the third line of (C.19) into (C.23) gives, for $8<e<$ 12.

$$
\begin{aligned}
\tilde{G}(e-i 0)= & \frac{1}{i \pi} \frac{6}{\pi^{2}}\left(L_{0}(12-e)+L_{2}^{-}(e-8)\right) \\
& -\frac{1}{(i \pi)^{3}}\left(L_{0}(12-e)+3 L_{2}^{-}(e-8)-3 L_{1}^{-}(e-4)+L_{0}(e)\right) \\
= & \frac{1}{i \pi^{3}}\left(7 L_{0}(12-e)+9 L_{2}^{-}(e-8)-3 L_{1}^{-}(e-4)+L_{0}(e)\right)
\end{aligned}
$$

Then applying (C.21) we obtain, for $8<e<12$,

$$
\begin{aligned}
\tilde{G}(e-i 0)= & \frac{1}{i \pi^{3}}\left[7 F_{3}(12-e)+9 F_{3}(e)+18 i \pi F_{2}(e-4)-9 \pi^{2} F_{1}(e-8)\right. \\
& \left.-3 F_{3}(e)-3 i \pi F_{2}(e-4)+F_{3}(e)\right]
\end{aligned}
$$

yielding

$$
\operatorname{Re} \tilde{G}(e-i 0)=\frac{15}{\pi^{2}} F_{2}(e-4)=\frac{15}{\pi^{2}} \int_{0}^{\infty} \exp (-(e-6) \lambda) K_{0}(2 \lambda)^{2} I_{0}(2 \lambda) d \lambda
$$

and

$$
\begin{aligned}
\operatorname{lm} \tilde{G}(e-i 0)= & -\frac{7}{\pi^{3}}\left(F_{3}(12-e)+F_{3}(e)\right)+\frac{9}{\pi} F_{1}(e-8) \\
= & -\frac{14}{\pi^{3}} \int_{0}^{\infty} \cosh (e-6) \lambda K_{0}(2 \lambda)^{3} d \lambda \\
& +\frac{9}{\pi} \int_{0}^{\infty} \exp (-(e-6) \lambda) K_{0}(2 \lambda) I_{0}(2 \lambda)^{2} d \lambda
\end{aligned}
$$


Finally we study $e>12$. This is most easily done by combining (C.27) with (C.23) to obtain

$$
\tilde{G}(e-i 0)=\frac{6}{i \pi^{3}}\left[L_{0}(12-e)+L_{2}^{-}(e-8)\right]-G(e-i 0) \quad(8<e<12)
$$

and applying (C.14) to the $L_{0}$ term to find, for $e>12$,

$$
\begin{aligned}
\tilde{G}(e-i 0)= & \frac{6}{i \pi^{3}}\left[-L_{3}^{-}(e-12)+L_{2}^{-}(e-8)\right]-G(e) \\
= & \frac{6}{i \pi^{3}}\left[-F_{3}(e)-3 i \pi F_{2}(e-4)+3 \pi^{2} F_{1}(e-8)+i \pi^{3} F_{0}(e-12)\right. \\
& \left.+F_{3}(e)+2 i \pi F_{2}(e-4)-\pi^{2} F_{1}(e-8)\right]+G(12-e)
\end{aligned}
$$

whence

$$
\operatorname{Re} \tilde{G}(e-i 0)=7 F_{0}(e-12)-\frac{6}{\pi^{2}} F_{2}(e-4)
$$

and

$$
\operatorname{lm} \tilde{G}(e-i 0)=-\frac{12}{\pi} F_{1}(e-8)<0
$$

Hence there is a cut from 12 to $\infty$ in the second sheet.

Collecting our results, we see that $\operatorname{Im} \bar{G}(e-i 0)$ is

$$
\begin{array}{cl}
\text { zero for } & e<0, \\
\text { positive for } & 0<e<8,
\end{array}
$$

monotonically decreasing for $8<e<12$, and negative for $12<e<\infty$.

Therefore there is just one point between 8 and 12 where $\tilde{G}$ is real: by (C.31), this takes place at $e_{*}-i 0$ where

$$
\frac{\int_{0}^{\infty} \cosh \left(e_{*}-6\right) \lambda K_{0}(2 \lambda)^{3} d \lambda}{\int_{0}^{\infty} e^{-\left(e_{*}-6\right) \lambda} K_{0}(2 \lambda) I_{0}(2 \lambda)^{2} d \lambda}=\frac{9 \pi^{2}}{14} .
$$

It follows from this and (C.30) that

$$
\tilde{G}_{*} \equiv \bar{G}\left(e_{*}\right)=\operatorname{Re} \tilde{G}\left(e_{*}\right)=\frac{15}{\pi^{2}} F_{2}\left(e_{*}-4\right)
$$


where $e_{*} \cong 8.43$ and $\tilde{G}_{*} \cong 1.56$.

Substituting (C.37) into the equation $Z\left(e_{*}\right)=0$, we find that $Z$ has a root at $e_{*}$ whenever

$$
e_{*}-6+\hat{g}+\frac{12}{\hat{f}}=\left[\hat{g} \frac{12}{\hat{f}}-\left(e_{*}-6\right)\left(e_{*}-6+\hat{g}\right)\right] \tilde{G}_{*}
$$

The locus of this equation is a rectangular hyperbola $h_{*}$ in the $\hat{g}-(12 / \hat{f})$ plane, concave upward with the vertical asymptote at $\left(\hat{g}=\frac{1}{\hat{G}_{0}}, 12 / \hat{f}=\infty\right)$ and horizontal asymptote at $\left(\hat{g}=\infty, 12 / \hat{f}=e_{*}-6+\tilde{G}_{*}^{-1}\right)$. Below $h_{*}, Z\left(e_{*}\right)$ is positive, above $h_{*}, Z\left(e_{*}\right)$ is negative.

We now study the roots of $Z(\hat{E})$ on the lower half-plane of the second sheet, by following the " $Z$-trajectory" traced in the complex $Z$-plane as $\hat{E}$ traces a large semi-circle encircling the lower half-plane counterclockwise, from $+\infty-i 0$ to $-\infty-i 0$ just below the real line and then back from $-\infty$ to $+\infty$ along an arc through large negative $\operatorname{Im} \hat{E}$. By Cauchy's theorem applied to $\frac{d}{d E} \ln Z$, the $Z$ trajectory will encircle the origin counterclockwise as many times as there are roots in the lower-half plane. Since our semi-circle excludes real roots, the $Z$-trajectory will execute a small half-circle clockwise around the origin every time a real root is encountered on the way from $\hat{E}=+\infty$ to $\hat{E}=-\infty$.

We may write (2.23) as

$$
\begin{aligned}
Z & =\left(\hat{E}-e_{+}\right)\left(\hat{E}-e_{-}\right) \mathcal{G}_{00}(\hat{E})+\hat{E}-e_{0} \\
& =(\hat{E}-6+\hat{g})\left[(\hat{E}-6) \mathcal{G}_{00}(\hat{E})+1\right]+\frac{12}{\hat{f}}\left(1-\hat{g} \mathcal{G}_{\infty}(\hat{E})\right)
\end{aligned}
$$

At $\hat{E} \rightarrow-\infty . \tilde{G}(\hat{E}) \sim \frac{1}{6-\hat{E}}(\ln (6-\hat{E}))^{2}$ as seen from the form of $F_{2}(8-\hat{E})$ in (C.20). Therefore the dominant term in $Z$ is $-\hat{E}(\ln (-\hat{E}))^{2}$, which makes an arc into the upper half-plane of $Z$, running from $Z=+\infty$ to $Z=-\infty$ as $\hat{E}$ goes from $-\infty$ to $+\infty$, and crossing the negative $Z$-axis because $\ln (-\hat{E})$ acquires a positive imaginary part. As $\hat{E}$ runs from $+\infty$ to 0 below the cut, $\operatorname{lm} Z=\left(\hat{E}-e_{+}\right)\left(\hat{E}-e_{-}\right) \operatorname{lm} \tilde{G}(\hat{E})$ starts out negative and changes sign at $e_{+}$, at $e_{*}$, and (if $e_{-}>0$ ) at $e_{-}$. Hence the $Z$-trajectory makes at most three excursions into the upper half-plane: the arc at $\hat{E}=\infty$, which contributes +1 to the winding number; an excursion between $e_{+}$and $e_{*}$; and, if $e_{-}>0$, an excursion between $e_{-}$and 0 . 
By (2.37) and (2.41)-(2.42), $Z$ has no negative real root on the physical sheet unless $e_{0}<0<\frac{e_{-}}{e_{0}}$, which implies $e_{-}<0$. Thus if $e_{-}>0$, the sign of $Z(0)$ agrees with that of $Z(-\infty)$ on the physical sheet, which is positive. Since $\tilde{G}(0)=G(0), Z(0)$ is also positive on the second sheet if $e_{-}>0$. But $Z\left(e_{-}\right)=e_{-}-e_{0}$ is also positive by (2.27). Hence the excursion between $e_{-}$and 0 can be reduced to the real line without changing the winding number.

The excursion between $e_{+}$and $e_{*}$ is ambiguous. We have $Z\left(e_{+}\right)=e_{+}-e_{0}>$ 0 , so that if $Z\left(e_{*}\right)>0$ this excursion can also be ignored. But if $Z\left(e_{*}\right)<0$ the excursion encircles the origin; since $\tilde{G}\left(e_{*}\right)>0$ by (C.37), and $e_{*}>6>e_{-}$, this can happen only if $e_{+}>e_{*}$, so that the excursion is counterclockwise.

Thus if $Z$ has no real negative roots on the second sheet, then there is one root in the lower half-plane due to the excursion at $\hat{E}=\infty$, and if $Z\left(e^{*}\right)<0$ a second root appears on account of the excursion at $e_{*}<\hat{E}<e_{+}$. But each real root for which $d Z / d \hat{E}<0$ produces an additional clockwise excursion lowering the winding number by 1 .

To follow the transformation of real to complex roots, let $\hat{g}$ decrease from $\infty$ to 0 while $\hat{f}$ is fixed at a value $\hat{f}>\hat{f}_{*}$ where

$$
\hat{f}_{*} \equiv \frac{12 \tilde{G}_{*}}{1+\left(e_{*}-6\right) \tilde{G}_{*}},
$$

so that $Z\left(e_{*}\right)>0$ for all $\hat{g}$. Then the excursion $\left(e_{*}<\hat{E}<e_{+}\right)$does not contribute to the winding number.

From (C.39) we have

$$
Z(0)=(\hat{g}-6)\left(1-6 \mathcal{G}_{\infty 0}(0)\right)+\frac{12}{f}\left(1-\hat{g} \mathcal{G}_{\infty}(0)\right)
$$

the same on both sheets. Since $\mathcal{G}_{00}(0)>0, Z(0)$ becomes negative for very large $\hat{g}$; but $Z(-\infty)>0$ on both sheets, and so there is a negative real root (bound state) on the physical sheet, and also one on the second sheet. As $\hat{E}$ moves from 0 to $-\infty$ below the real line, $Z(\hat{E})$ moves from $Z(0)$ to $+\infty$ passing above the origin so that the excursion to the upper half-plane at infinite $\hat{E}$ is neutralized; the winding number is zero and there is no complex root.

As $\hat{g}$ decreases, $Z(0)$ increases monotonically (since $6 \mathcal{G}_{00}(0)>1$ ) and is manifestly positive when $\hat{g}=0$. Hence for some $\hat{g}, Z(0)$ becomes positive, the bound state disappears, and the physical root passes through $\hat{E}=0$ to the second 
sheet. Now the second sheet contains two negative real roots, so that $Z$ must become negative for some $\hat{E}<0$; hence the $Z$-trajectory makes a full clockwise circle about the origin while passing from $Z(0)$ to $Z(-\infty)$. This again nullifies the arc at $\hat{E}=\infty$ so that there are no complex roots.

As $\hat{g}$ decreases further, the two roots move toward each other, meet, and separate in the imaginary direction. (This must happen because (C.39) shows that $Z(\hat{E})>0$ for all $\hat{E}<0$ if $\hat{g}=0$.) The disappearance of the real roots means that $Z$ no longer dips below the origin. Thus the clockwise encirclement is lost, the arc at $\infty$ is no longer neutralized, and there is now one root in the lower half of the second sheet, the other having moved to the upper half.

This picture agrees entirely with that described for $\hat{f}>0$ in Section (2.3), using the "continuum approximation" (2.48)-(2.49). But we now find that for $\hat{f}<\hat{f}_{*}$ the second sheet has an extra root in the lower half-plane for large $\hat{g}$, but not for small $\hat{g}$. As $\hat{g}$ decreases passing through the hyperbola $h_{*}$, the sign of $Z\left(e_{*}\right)$ changes and the extra root disappears through the cut at $e_{*}$ into a more remote sheet (not into the physical sheet, since $e_{*}>4$ ).

We should also mention that when $\hat{f} \rightarrow 0$ the second-sheet root corresponding to $y_{2}$ in Section 2.3 (following Eq. (2.58)) does not disappear to infinity as implied by (i) and (iii) stated there. The difference arises because $\tilde{G}(\hat{E}) \rightarrow 0$ as $\hat{E} \rightarrow-\infty$. whereas in the continuum approximation $\tilde{G}(\hat{E})$ grows as $\sqrt{\frac{|\hat{E}|}{2 \pi}}$ : see (2.63) for $y \rightarrow-\infty, \alpha_{1}=\alpha_{2}=\frac{\pi}{2}$.

To summarize, the second sheet may contain from 1 to 4 roots of $Z$, according to the following cases.

I: $\quad Z\left(e_{*}\right)>0, Z(0)<0$ (bound state): one root on second sheet, must be negative real.

II: $Z\left(e_{*}\right)>0, Z(0)>0$ (no bound state): two roots on second sheet, either both negative real or a conjugate complex pair.

III: $Z\left(e_{*}\right)<0, Z(0)<0$ (bound state): three roots on the second sheet, one negative real and two conjugate complex.

IV: $Z\left(e_{*}\right)<0, Z(0)>0$ (no bound state): four roots on second sheet; either two negative real plus a conjugate pair, or two conjugate pairs.

There can be no additional real roots in cases I and II because each pair would induce a clockwise encirclement of the origin, making the winding number negative 
which is impossible because $Z(\hat{E})$ has no poles. In cases III and IV our arguments do not exclude the possibility that the "extra" pair is also found on the negative real line, inducing a clockwise encirclement that cancels the arc from $Z\left(e_{+}\right)$to $Z\left(e_{*}\right)$; but this would require an improbable migration in view of further description below. There can never be more than the stated number of roots on the whole second sheet.

In the $\hat{g}-(12 / \hat{f})$ plane the four regions are separated by the two hyperbolas $h_{*}$, defined by $(C .38)$, and $h_{0}$, defined by $Z(0)=0$ or

$$
\hat{g}-6+\frac{12}{\hat{f}}=\left[6(\hat{g}-6)+\hat{g} \frac{12}{\hat{f}}\right] \hat{g}_{0} .
$$

The curve $h_{0}$ is also a rectangular hyperbola, concave upward in the $\hat{g}-(12 / \hat{f})$ plane, and has $\hat{g}$-intercept $(\hat{g}=6,12 / \hat{f}=0)$ and vertical asymptote $\left(\hat{g}=g_{0}^{-1}\right.$. $12(\hat{f}=\infty)$.

On account of (C.23), since $G(0)=\tilde{G}(0)=g_{0}$, we have

$$
g_{0}=\frac{3}{\pi^{2}} F_{2}(8)<\frac{3}{\pi^{2}} F_{2}\left(e_{*}-4\right)
$$

since $e_{*}<12$ and $F_{2}$ is a decreasing function by (C.20). Comparing with (C.37), we find

$$
\frac{1}{g_{0}}>5 \frac{1}{\tilde{G}_{*}}
$$

so that $h_{0}$ intersects the $\hat{g}$-axis to the right of $h_{*}$. This establishes Region IV as lying between the upper segments of $h_{0}$ and $h_{*}$ and open to $12 / \hat{f} \rightarrow \infty$, and shows that $h_{0}$ and $h_{*}$ intersect at some point $(\bar{g}, 12 / \bar{f})$. (See Figure 11.)

We can now describe the migration of the roots as the potential well becomes shallow. First suppose that the well has high walls: $\hat{f}$ very large. Then we expect a good resonance. Indeed, we see that as $\hat{f} \rightarrow \infty$, (C.39) has a root on each sheet near $\hat{E}=6-\hat{g}$. This root is real for large $\hat{g}$, but as $\hat{g}$ decreases through 6 the physical root passes through $\hat{E}=0$ to the second sheet, and the two roots pass along either side of the cut on the second sheet, up to $\hat{E}=6$; the root below the cut represents the expected resonance. (The factor $\left[(\hat{E}-6) \mathcal{G}_{00}(\hat{E})+1\right]$ evidently cannot vanish on either sheet, as we have proved there are no other roots for large $\hat{f}$.)

If $\hat{f}$ is only moderately large, the two roots no longer coincide on the two sheets; the physical root reaches $\hat{E}=0$ first and must turn back on the second 
sheet to meet the other. The migration of the complex root toward $\hat{E} \approx 6$ below the cut then follows a trajectory with a significant negative imaginary part, representing the width of the resonance.

Now let $\hat{f}$ be very small. By neglecting the first term of (C.39) we find a root at $\mathcal{G}_{00}(\hat{E})=\frac{1}{\hat{g}}$. which for $\hat{g}>g_{0}^{-1}$ defines $\hat{E}$ as real and negative on both sheets, but more negative on the second sheet. When $\hat{g}$ descends below $g_{0}^{-1}$. the physical root turns back onto the second sheet, and the two roots meet when $\hat{g}=1 / \max _{\hat{E}<0} \tilde{G}(\hat{E}) \cong 3.096$. They then become a complex conjugate pair, each migrating along a trajectory defined by $\operatorname{lm} \tilde{G}=0$ until (at $\hat{g}=\frac{1}{G_{*}}$ ) it reaches $e_{*}$ and disappears through the cut.

There is no doubt that this happens for sufficiently small $\hat{f}$, because some root must disappear through the cut, and the only other root in each half-plane is found by taking $\hat{E}$ very large and positive. In that limit (C.39) is approximated on the second sheet by

$$
\hat{E}^{2} \tilde{G}(\hat{E})+\frac{12}{\hat{f}} \cong-\hat{E}(\ln (-\hat{E}))^{2}+\frac{12}{\hat{f}},
$$

where in the lower half-plane $\ln (-\hat{E})$ acquires a positive imaginary part. Thus by giving $\hat{E}$ a suitable negative imaginary part we make $\hat{E}(\ln (-\hat{E}))^{2}$ real, and its magnitude can be adjusted to make (C.44) vanish. Thus the "extra" root stays far away at $+\infty$ for all $\hat{g}$, and the one that disappears into $e_{*}$ must have migrated from the negative real axis.

On the other hand, we could have raised $\hat{f}$ gradually while keeping $\hat{g}$ very large. Then the bound state would persist on the physical sheet, and the real root on the second sheet would have no opportunity to become complex. Consequently as $\hat{f}$ increases past $\hat{f}_{*}$ it is the root from $+\infty$ that must disappear into the cut.

These examples show that we cannot really determine which is the "extra" root. Either root can be sent to $e_{*}$, depending on the path chosen in the $\hat{g}-(12 / \hat{f})$ plane.

We may sharpen this observation by tracing the boundary of Region IV. We begin at $(\hat{g}=\bar{g}, 12 / \hat{f}=12 / \bar{f})$ where $Z(0)=Z\left(e_{*}\right)=0$. The four roots are at 0 , at some negative real $\hat{E}$, and at $e_{*}$ on each side of the cut. Passing along $h_{*}$ to $\left(\hat{g}=\frac{1}{\hat{G}_{*}}, 12 / \hat{f}=\infty\right)$ we see the two real roots move together and migrate as a conjugate pair to $+\infty$, while the root at $e_{*}$ does not move. Now increasing $\hat{g}$ to $g_{0}^{-1}$ with $\hat{f}$ fixed at zero, the roots at $+\infty$ stay put while the pair at $e_{*} \pm i 0$ 
migrates to the negative real axis, separates and one member moves to zero. Finally we move along $h_{0}$ to $(\hat{g}=\bar{g}, 12 / \hat{f}=12 / \bar{f})$; the real roots remain on the negative real axis while the ones at $+\infty$ come to $e_{*}$. The two pairs of roots have been exchanged.

It follows that somewhere within Region IV is a critical point $(\hat{g}, 12 / \hat{f})$ at which a double root exist in each half-plane. Circling this point causes two single roots to change places.

An alternative scenario not excluded by our arguments is that the roots from $+\infty$ visit the negative real axis on the way to $e_{*}$. In this case the critical $(\hat{g}, 12 / \hat{f})$ might yield a quadruple zero at negative real $\hat{E}$. 


\section{APPENDIX D}

In this appendix, we shall establish the relationship between the four-point function (2.15) for the scattering of two fermions, derived from the Hamiltonian $h$ of (1.22), and the corresponding four-point function (3.43) from the equivalent boson-fermion Hamiltonian $H$ of (1.30) when $\xi_{0}, \eta_{0}$ and $\zeta_{0}$ of (1.31) become $+\infty$. (Each of these functions is characterized by four "momentum-points" $\frac{\vec{p}}{2} \pm \vec{p}$ and $\frac{\vec{P}}{2} \pm \vec{q}$.)

We first generalize the four-point function (2.15) off the mass shell ( $E=$ $\left.\epsilon_{\frac{\beta}{2}+\vec{p}}+\epsilon_{\frac{p}{2}-\vec{p}}\right)$ through the replacement of $E$ in the matrix $\mathcal{G}$ by an independent variable $P_{0}$. The generalized four-point function

$$
\left(\vec{q}\left|\gamma\left(P_{0}, \vec{P}\right)\right| \vec{p}\right)=2 t \tilde{u}(\vec{q}) \mathcal{F}^{-1}\left(P_{0}, \vec{P}\right) u(\vec{p}),
$$

with $u$ given by (2.12) and $\mathcal{F}$ by (3.25), can be induced by a source term

$$
h_{\chi}=\sum_{j}\left(\bar{\chi}_{j \sigma} \alpha_{j \sigma}+\alpha_{j \sigma}^{\dagger} \chi_{j \sigma}\right)
$$

added to the Hamiltonian $h$ of (1.22), where the external sources $\chi, \bar{\chi}$ are Grassmann numbers, ${ }^{8}$ which are anticommuting with each other and with fermion operators, but commuting with boson operators. $\chi_{j \sigma}$ and $\bar{\chi}_{j \sigma}$ are arbitrary functions of time $t$, subject to the condition that $\lim _{t \rightarrow \pm \infty} \chi_{j \sigma}=\lim _{t \rightarrow \pm \infty} \bar{\chi}_{j \sigma}=0$. Let $\mid g d)$ be the ground state of $h$. Assume the state to be $\mid g d)$ at $t=-\infty$. Under the action of the source term (D.2), the state will change in time; the probability amplitude for the system to remain in the same state $\mid g d)$ at $t=+\infty$ is

$$
(g d|U(\infty,-\infty)| g d)=e^{W}
$$

where

$$
U\left(t_{2}, t_{1}\right)=T e^{-i \int_{t_{1}}^{t_{2}} d t h_{x}(t)}
$$

with $T$ denoting the time-ordering product. The time development of the operators in $h_{\chi}(t)$ is given by

$$
O(t)=e^{i h t} O(0) e^{-i h t} .
$$

The logarithm of the probability amplitude $W$ in (D.3) can be expanded according to the powers of $\chi$ and $\bar{\chi}$; i.e. 


$$
W=\sum_{n} W^{(n)}
$$

with $W^{(n)}$ representing the sum of all connected diagrams, each with $n$ incoming fermion lines all stemming from $\chi$ 's and $n$ outgoing lines all ending at $\bar{\chi}$. The vertex at which the external source creates, or annihilates, a fermion of energy $p_{0}$. momentum $\vec{p}$ and spin $\sigma$ gives a factor $-i \chi_{\vec{p}, \sigma}\left(p_{0}\right)$, or $-i \bar{\chi}_{\bar{p}, \sigma}\left(p_{0}\right)$, where

$$
\chi_{\bar{p}, \sigma}\left(p_{0}\right)=\sum_{j} \int_{-\infty}^{\infty} d t e^{i p_{0} t-i \vec{p} \cdot \bar{r}_{j}} \chi_{j \sigma}(t)
$$

and

$$
\bar{\chi}_{\vec{p}, \sigma}\left(p_{0}\right)=\sum_{j} \int_{-\infty}^{\infty} d t e^{-i p_{0} t+i \bar{p} \cdot \bar{r}_{j}} \bar{\chi}_{j \sigma}(t)
$$

For $n=2$, the diagrams corresponding to $W^{(2)}$ are shown in Figure 12 in which the bare vertex with two fermions in and two fermions out gives rise to, in accordance with the Hamiltonian (1.22), a factor $-i \cdot 2 t \sum_{\mu} \mathcal{K}_{\mu} u_{\mu}(\vec{q}) u_{\mu}(\vec{p})$ with $\mathcal{K}_{\mu}$ given by the $\mu^{\text {th }}$ diagonal element of (2.14) and $\vec{p}, \vec{q}$ denoting the initial and final relative momenta. It follows that

$$
\begin{aligned}
W^{(2)}=\ell^{9} & \int \frac{d^{4} p_{1}}{(2 \pi)^{4}} \int \frac{d^{4} p_{2}}{(2 \pi)^{4}} \int \frac{d^{4} q_{1}}{(2 \pi)^{4}} \int \frac{d^{4} q_{2}}{(2 \pi)^{4}}(2 \pi)^{4} \\
& \cdot \delta^{3}\left(\vec{p}_{1}+\vec{p}_{2}-\vec{q}_{1}-\vec{q}_{2}\right) \delta\left(p_{10}+p_{20}-q_{10}-q_{20}\right) \\
& \cdot \bar{\chi}_{\vec{p}_{2} \downarrow}\left(p_{20}\right) \bar{\chi}_{\vec{p}_{1} \uparrow}\left(p_{10}\right) \chi_{\vec{q}_{1} \uparrow}\left(q_{10}\right) \chi_{\vec{q}_{2} \downarrow}\left(q_{20}\right) G\left(p_{1}, p_{2} ; q_{1}, q_{2}\right)
\end{aligned}
$$

with

$G\left(p_{1}, p_{2} ; q_{1}, q_{2}\right)=\frac{(-i)\left(\vec{q}\left|\gamma\left(P_{0}, \vec{P}\right)\right| \vec{p}\right)}{\left(p_{10}-\epsilon_{\vec{p}_{1}}+i 0+\right)\left(p_{20}-\epsilon_{\vec{p}_{2}}+i 0+\right)\left(q_{10}-\epsilon_{\vec{q}_{1}}+i 0+\right)\left(q_{20}-\epsilon_{\overrightarrow{q_{2}}}+i 0+\right)}$

where $d^{4} p \equiv d p_{0} d^{3} \vec{p}, \vec{P}=\vec{p}_{1}+\vec{p}_{2}=\vec{q}_{1}+\vec{q}_{2}, \quad P_{0}=p_{10}+p_{20}=q_{10}+q_{20}, \vec{p}=$ $\frac{1}{2}\left(\vec{p}_{1}-\vec{p}_{2}\right)$ and $\vec{q}=\frac{1}{2}\left(\vec{q}_{1}-\vec{q}_{2}\right)$. Therefore, the off-mass-shell four-point function (D.1) is the amputated part (the part with all the fermion propagators connecting to the external source removed) of the diagrams in Fig. 12.

Now we come to the boson-fermion Hamiltonian $H$ of (1.30). Corresponding to (D.2), we add a term 


$$
H_{\chi}=\sum_{j}\left(\bar{\chi}_{j \sigma} a_{j \sigma}+a_{j \sigma}^{\dagger} \chi_{j \sigma}\right)+\sum_{j} \epsilon_{\sigma \sigma^{\prime}}\left(b_{j}^{\dagger} a_{j \sigma^{\prime}} \chi_{j \sigma}+\bar{\chi}_{j \sigma} a_{j \sigma^{\prime}}^{\dagger} b_{j}\right)
$$

to the Hamiltonian (1.30), where $\epsilon_{\uparrow \downarrow}=-\epsilon_{\downarrow \uparrow}=1$ and $\epsilon_{\uparrow \uparrow}=\epsilon_{\downarrow \downarrow}=0$. The probability amplitude for the system, which is in the ground state of $H, \mid g d>$ at $t=-\infty$, to remain in the same state at $t=+\infty$ is

$$
<g d\left|U^{\prime}(\infty,-\infty)\right| g d>=e^{W^{\prime}}
$$

with

$$
U^{\prime}\left(t_{2}, t_{1}\right)=T e^{-i \int_{t_{1}}^{t_{2}} d t H_{x}(t)}
$$

The time development of the operators in $H_{\chi}(t)$ is given by

$$
O(t)=e^{i H t} O(0) e^{-i H t}
$$

Similar to (D.6), $W^{\prime}$ can also be expanded according to the powers of $\chi$ and $\bar{\chi}$. i.e.

$$
W^{\prime}=\sum_{n} W^{\prime(n)}
$$

with $W^{\prime(n)}$ corresponding to the sum of all connected diagrams with $n \chi$-dependent vertices and $n \bar{\chi}$-dependent vertices. These diagrams are complicated by the observation that $\chi, \bar{\chi}$ in (D.11) can also induce transitions between fermions and bosons. The vertex at which a fermion of energy $p_{0}$, momentum $\vec{p}$ and spin $\sigma$ is turned into a boson of energy $P_{0}$ and momentum $\vec{P}$ via $\chi$ contributes a factor $-i \epsilon_{\sigma^{\prime} \sigma} \chi_{P-\vec{p}, \sigma^{\prime}}\left(P_{0}-p_{0}\right)$, and the vertex at which a boson of energy $P_{0}$ and momentum $\vec{P}$ is turned into a fermion of energy $p_{0}$. momentum $\vec{p}$ and spin $\sigma$ via $\bar{\chi}$ contributes a factor $-i \epsilon_{\sigma \sigma^{\prime}} \bar{\chi}_{\vec{P}-\bar{p}_{1} \sigma^{\prime}}\left(P_{0}-p_{0}\right)$, with the definitions of these Fourier transforms given by (D.7)-(D.8). Following the same strategy as in Section 1 , it can be shown that with the same external sources $\chi$ and $\bar{\chi}$, the Hamiltonian $h+h_{\chi}$ and the finite energy sector of the Hamiltonian $H+H_{\chi}$ are equivalent upon sending $\xi_{0}, \eta_{0}$ and $\zeta_{0}$ of $(1.31)$ to $+\infty$. Therefore, we expect that

$$
W^{(n)}=W^{\prime(n)}
$$


In the following, we prove this equality explicitly for the case $n=2$. The diagrams corresponding to $W^{\prime(2)}$ are shown in Fig. 12(b) of which only the last two give rise to the vertex function $\left\langle\vec{q}\left|\Gamma\left(P_{0}, \vec{P}\right)\right| \vec{p}\right\rangle$ of (3.43), as we shall see. It follows that

$$
\begin{aligned}
W^{\prime}(2)=\ell^{9} & \int \frac{d^{4} p_{1}}{(2 \pi)^{4}} \int \frac{d^{4} p_{2}}{(2 \pi)^{4}} \int \frac{d^{4} q_{1}}{(2 \pi)^{4}} \int \frac{d^{4} q_{2}}{(2 \pi)^{4}}(2 \pi)^{4} \\
& \cdot \delta^{3}\left(\vec{p}_{1}+\vec{p}_{2}-\vec{q}_{1}-\vec{q}_{2}\right) \delta\left(p_{10}+p_{20}-q_{10}-q_{20}\right) \\
& \cdot \bar{\chi}_{\overrightarrow{p_{2} \downarrow}}\left(p_{20}\right) \bar{\chi}_{\vec{p}_{1} \uparrow}\left(p_{10}\right) \chi_{\vec{q}_{1} \uparrow}\left(q_{10}\right) \chi_{\vec{q}_{2} \downarrow}\left(q_{20}\right) G^{\prime}\left(p_{1}, p_{2} ; q_{1}, q_{2}\right)
\end{aligned}
$$

where

$$
G^{\prime}\left(p_{1}, p_{2} ; q_{1}, q_{2}\right)=\frac{\left.(-i)<\vec{q}\left|\gamma^{\prime}\left(P_{0}, \vec{P}\right)\right| \vec{p}\right\rangle}{\left(p_{10}-\epsilon_{\vec{p}_{1}}+i 0+\right)\left(p_{20}-\epsilon_{\vec{p}_{2}}+i 0+\right)\left(q_{10}-\epsilon_{\vec{q}_{1}}+i 0+\right)\left(q_{20}-\epsilon_{\bar{q}_{2}}+i 0+\right)}
$$

and we have isolated out the amputated part, $\left\langle\vec{q}\left|\gamma^{\prime}\left(P_{0}, \vec{P}\right)\right| \vec{p}\right\rangle$, in the same manner as in (D.10) for the sake of comparison. Written in detail, it is

$$
\left.<\vec{q}\left|\gamma^{\prime}\left(P_{0}, \vec{P}\right)\right| \vec{p}\right\rangle=A+B
$$

where

$$
\begin{aligned}
& A=(-i)\left[P_{0}-\epsilon_{\frac{P}{2}+\vec{q}}-\epsilon_{\frac{P}{2}-\vec{q}}+<\vec{q}\left|V\left(P_{0}, \vec{P}\right)\right| b>\right] \\
& \cdot\left[P_{0}-\epsilon_{\frac{\vec{P}}{2}+\vec{p}}-\epsilon_{\frac{\vec{p}}{2}-\vec{p}}+<\vec{p}\left|V\left(P_{0}, \vec{P}\right)\right| b>\right] D\left(P_{0}, \vec{p}\right), \\
& B=\left\langle\vec{q}\left|\hat{\Gamma}\left(P_{0}, \vec{P}\right)\right| \vec{p}\right\rangle=2 t \tilde{u}(\vec{q}) \hat{\mathcal{F}}\left(P_{0}, \vec{P}\right) u(\vec{p})
\end{aligned}
$$

with $\left\langle\vec{q}\left|V\left(P_{0}, \vec{P}\right)\right| b\right\rangle$ given by (3.41), $D\left(P_{0}, \vec{P}\right)$ by (3.24) and $\hat{\mathcal{F}}$ by (3.21). Introducing a $4 \times 1$ column matrix

$$
W(\vec{P})=\left(\begin{array}{c}
0 \\
w
\end{array}\right)
$$

with $w$ the $3 \times 1$ column matrix in (3.26), we have

$$
\epsilon_{\frac{\beta}{2}+\vec{q}}+\epsilon_{\frac{p}{2}-\vec{q}}=2 t(6-2 \tilde{u}(\vec{q}) W(\vec{P}))
$$

and

$$
\left\langle\vec{q}\left|V\left(P_{0}, \vec{P}\right)\right| b\right\rangle=-\frac{4 t}{\hat{f}} \tilde{u}(\vec{q}) \hat{\mathcal{F}}^{-1}\left(P_{0}, \vec{P}\right) W(\vec{P})
$$


With the aid of the identities (2.16), we find that

$$
\begin{gathered}
P_{0}-\epsilon_{\frac{p}{2}+\vec{q}}-\epsilon_{\frac{p}{2}-\vec{q}^{+}}+\left\langle\vec{q}\left|V\left(P_{0}, \vec{P}\right)\right| b>=-2 t \sum_{\mu} u_{\mu}(\vec{q})\left[\hat{\mathcal{F}}^{-1}\left(P_{0}, \vec{P}\right)\right]_{\mu 0}\right. \\
=-2 t \frac{\operatorname{det} \mathcal{F}\left(P_{0}, \vec{P}\right)}{\operatorname{det} \hat{\mathcal{F}}\left(P_{0}, \vec{P}\right)} \sum_{\mu} u_{\mu}(\vec{q})\left[\hat{\mathcal{F}}^{-1}\left(P_{0}, \vec{P}\right)\right]_{\mu 0}
\end{gathered}
$$

and $A$ is simplified to be

$$
A=2 t \frac{\operatorname{det} \mathcal{F}\left(P_{0}, \vec{P}\right)}{\hat{g} \operatorname{det} \hat{\mathcal{F}}\left(P_{0}, \vec{P}\right)} \sum_{\mu} u_{\mu}(\vec{q})\left[\mathcal{F}^{-1}\left(P_{0}, \vec{P}\right)\right]_{\mu 0} \sum_{\nu}\left[\mathcal{F}^{-1}\left(P_{0}, \vec{P}\right)\right]_{0_{\nu}} u_{\nu}(\vec{p}) \text {. }
$$

Using the algebraic identity

$$
\left(\hat{\mathcal{F}}^{-1}\right)_{\mu \nu}=\left(\mathcal{F}^{-1}\right)_{\mu \nu}-\frac{\operatorname{det} \mathcal{F}}{\hat{g} \operatorname{det} \hat{\mathcal{F}}}\left(\mathcal{F}^{-1}\right)_{\mu 0}\left(\mathcal{F}^{-1}\right)_{0 \nu}
$$

we end up with

$$
\left\langle\vec{q}\left|\gamma^{\prime}\left(P_{0}, \vec{P}\right)\right| \vec{p}\right\rangle=\left(\vec{q}\left|\gamma\left(P_{0}, \vec{P}\right)\right| \vec{p}\right)
$$

as expected. If we impose the on-mass-shell condition

$$
P_{0}=\epsilon_{\frac{p}{2}+\vec{p}}+\epsilon_{\frac{p}{2}-\vec{p}}=\epsilon_{\frac{p}{2}+\vec{q}}+\epsilon_{\frac{p}{2}-\vec{q}},
$$

the sum of the first eight diagrams of Figure 12, upon amputation, is proportional to

$$
\begin{aligned}
& \left(P_{0}-\epsilon_{\frac{\beta}{2}+\vec{q}}-\epsilon_{\frac{P}{2}-\vec{q}}\right)\left(P_{0}-\epsilon_{\frac{\beta}{2}+\vec{p}}-\epsilon_{\frac{P}{2}-\vec{p}}\right)+\left(P_{0}-\epsilon_{\frac{\beta}{2}+\vec{q}}-\epsilon_{\frac{\beta}{2}-\vec{q}}\right)<\vec{p}\left|V\left(P_{0}, \vec{P}\right)\right| b> \\
& \left.\quad+\left(P_{0}-\epsilon_{\frac{\beta}{2}+\vec{p}}-\epsilon_{\frac{\vec{p}}{2}-\vec{p}}\right)<\vec{q}\left|V\left(P_{0}, \vec{P}\right)\right| b\right\rangle
\end{aligned}
$$

and vanishes on account of (D.29). Therefore $\left\langle\vec{q}\left|\gamma^{\prime}\left(P_{0}, \vec{P}\right)\right| \vec{p}\right\rangle$ reduces to $\left\langle\vec{q}\left|\Gamma\left(P_{0}, \vec{P}\right)\right| \vec{p}\right\rangle$ of (3.43) only on the mass shell and (D.28) implies the equality between the $S$-matrix elements for two-fermion scattering derived from the Hamiltonian (1.22) and that from the Hamiltonian (1.30), as expected from the equivalence of these two Hamiltonians when $\xi_{0}, \eta_{0}$ and $\zeta_{0}$ of (1.31) becomes $+\infty$. 


\section{REFERENCES}

1. R. Friedberg, T. D. Lee and H. C. Ren, Ann.Phys. 228, 52 (1993).

2. P. Nozieres and S. Schmitt-Rink, J.Low Temperature Physics 59, 195 (1985).

3. R. Micnas, J. Ranninger and S. Robaszkiewicz, Rev.Mod.Phys. 62, 113 (1990) and the references cited therein.

4. R. Friedberg and T. D. Lee, Phys.Lett. 138A, 423 (1989), Phys.Rev. B40, 6745 (1989).

5. R. Friedberg. T. D. Lee and H. C. Ren, Phys.Rev. B45, 10732 (1992), B46, 14150 (1992).

6. G. N. Watson, Quart.J.Math. 10, 266 (1939).

7. L. Van Hove, Phys.Rev. 89, 1189 (1953).

8. See for example, T. D. Lee, Particle Physics and Introduction to Field Theory (Chur, Harwood Academic Publishers, 1981). 


\section{FIGURE CAPTIONS}

Figure 1. Family of hyperbolas given by (2.44), with the solid curve for $I_{0}=$ $g_{0}^{-1}=3.9568$ (the boundary of a bound state), the dashed curve for $e_{-}=0$ ( $I_{0}=$ $0)$, and the straight line for $e_{0}=0 \quad\left(I_{0}=\infty\right)$. The dotted curve is the trajectory $I_{0}=I_{c}$ given by (2.73)-(2.75), when the two roots of (2.70) coalesce on the second unphysical sheet. For $f>0$, there is at most one bound state, which exists only in the slanted shaded region. For $f<0$ and $e_{0}<0$, there are two bound states if $I_{0}>g_{0}^{-1}$ (vertically shaded region) and only one bound state if $I_{0}<g_{0}^{-1}$ (horizontally shaded region). All states are $s$-wave.

Figure 2. The upper half $z$-plane corresponds to the physical $E$-sheet and the lower half $z$-plane to the second unphysical $E$-sheet. The two wavy lines along the $x$-axis are the cuts in $\mathcal{G}(z)$, given by (2.55). The points $z=i y_{1}$ and $i y_{2}$ are the roots of (2.70), and $z=i y_{c}$ is where these two roots coalesce.

Figure 3. The solid curves are $I(i y)$ vs $y$ and the dashed curves are $\mathcal{G}(i y)^{-1}$ vs. $y$ (see (2.65)-(2.66)); their intersections are the roots of (2.70). (a) refers to the bound state case when $\hat{f}=0$ and $I(i y)=\hat{g}>g_{0}^{-1}$. (b) gives an example of the bound state case when $\hat{f}>0$ and $\hat{g}>6$ (therefore $\partial^{2} I(i y) / \partial y^{2}$ is positive at $y=0$ ). (c) is an example of no bound state, with both roots $y_{1}$ and $y_{2}$ negative. In both (b) and (c), the solid curve has three segments, each in the regions $y<-\sqrt{-e_{0}},-\sqrt{-e_{0}}<y<\sqrt{-e_{0}}$ and $y>\sqrt{-e_{0}}$.

Figure 4. The trajectories of the poles $z= \pm x_{r}+i y_{r}$ as a function of $12 / \hat{f}$ at $\hat{g}=5$; the one with $+x_{r}>0$ is the resonance pole. The solid line is for $0<12 / \hat{f}<12 / \hat{f}_{c} \cong 2$ and the dashed line is for $12 / \hat{f}<0$. The lower half $z$-plane corresponds to the second unphysical sheet.

Figure 5. The solid and dashed lines are the bare fermion and boson propagators. The four-point and three-point vertices are given by (3.8)-(3.12). 
Figure 6. (a) gives the integral equation (3.13) for $\left\langle\vec{q}\left|\hat{\Gamma}\left(P_{0}, \vec{P}\right)\right| \vec{p}\right\rangle$; (b) is the dressed boson propagator $D\left(P_{0}, \vec{P}\right)$ given by $(3.19)$ and $(\mathrm{c})$ denotes $-i \Pi\left(P_{0}, \vec{P}\right)$. the sum of all one-particle irreducible boson propagator diagrams (with the external lines amputated). The spin indices and some of the momentum labels are often omitted on the right-hand sides of these, as well as the following figures.

Figure 7. (a) gives the dressed vertex function $-i\left\langle b\left|V\left(P_{0}, \vec{P}\right)\right| \vec{p}\right\rangle$ of (3.41) and (b) is the full two-fermion scattering function $\left\langle\vec{q}\left|\Gamma\left(P_{0}, \vec{P}\right)\right| \vec{p}\right\rangle$ of (3.43).

Figure 8. (a) refers to the bare boson-fermion scattering vertex (4.2); (b) is the sum $-i<\vec{q}\left|\hat{\mathcal{S}}\left(P_{0}, \vec{P}\right)\right| \vec{p}>$ of all chain diagrams, and (c) gives the sum $-i<\vec{q}\left|\mathcal{S}\left(P_{0}, \vec{P}\right)\right| \vec{p}>$ of all boson-fermion scattering diagrams.

Figure 9. (a) refers to the bare boson-boson scattering vertex (4.12); (b) is the sum $<-i \dot{\vec{q}}\left|\hat{T}\left(P_{0}, \vec{P}\right)\right| \vec{p}>$ of all chain diagrams, and (c) gives the sum $-i<\vec{q}\left|T\left(P_{0}, \vec{P}\right)\right| \vec{p}>$ of all boson-boson scattering diagrams.

Figure 10. Solutions of the secular equation (B.12). The solid line, consisting of $\mathcal{N}+1$ segments, represents $\mathcal{G}_{\infty}$ vs. $\hat{E}$ and the dashed line, consisting of three segments, is $I^{-1}$ vs. $\hat{E}$ : (a) refers to the case $e_{-}<e_{1}$ and $e_{n}<e_{+}<e_{n+1}$; (b) denotes the case $e_{m}<e_{-}<e_{m+1}$ and $e_{n}<e_{+}<e_{n+1}$.

Figure 11. The four regions, I-IV, analysed in Appendix $C$, with each region corresponding to a particular case of the poles of the exact two-fermion scattering amplitude on the second sheet: I: $Z\left(e_{*}\right)>0, Z(0)<0$ : one root on second sheet, must be negative real. II: $Z\left(e_{*}\right)>0, Z(0)>0$ : two roots on second sheet, either both negative real or a conjugate complex pair. III: $Z\left(e_{*}\right)<0$, $Z(0)<0$ : three roots on the second sheet, one negative real and two conjugate complex. IV: $Z\left(e_{*}\right)<0, Z(0)>0$ : four roots on second sheet; either two negative real plus a conjugate pair, or two conjugate pairs.

Figure 12. (a) gives the four point function $W^{(2)}$, given by (D.9) and (D.10). (b) gives the four point function $W^{\prime(2)}$, given by (D.17) and (D.18). Here $1=$ $\vec{p}_{1}, 2=\vec{p}_{2}, 1^{\prime}=\vec{q}_{1}$ and $2^{\prime}=\vec{q}_{2}$. 


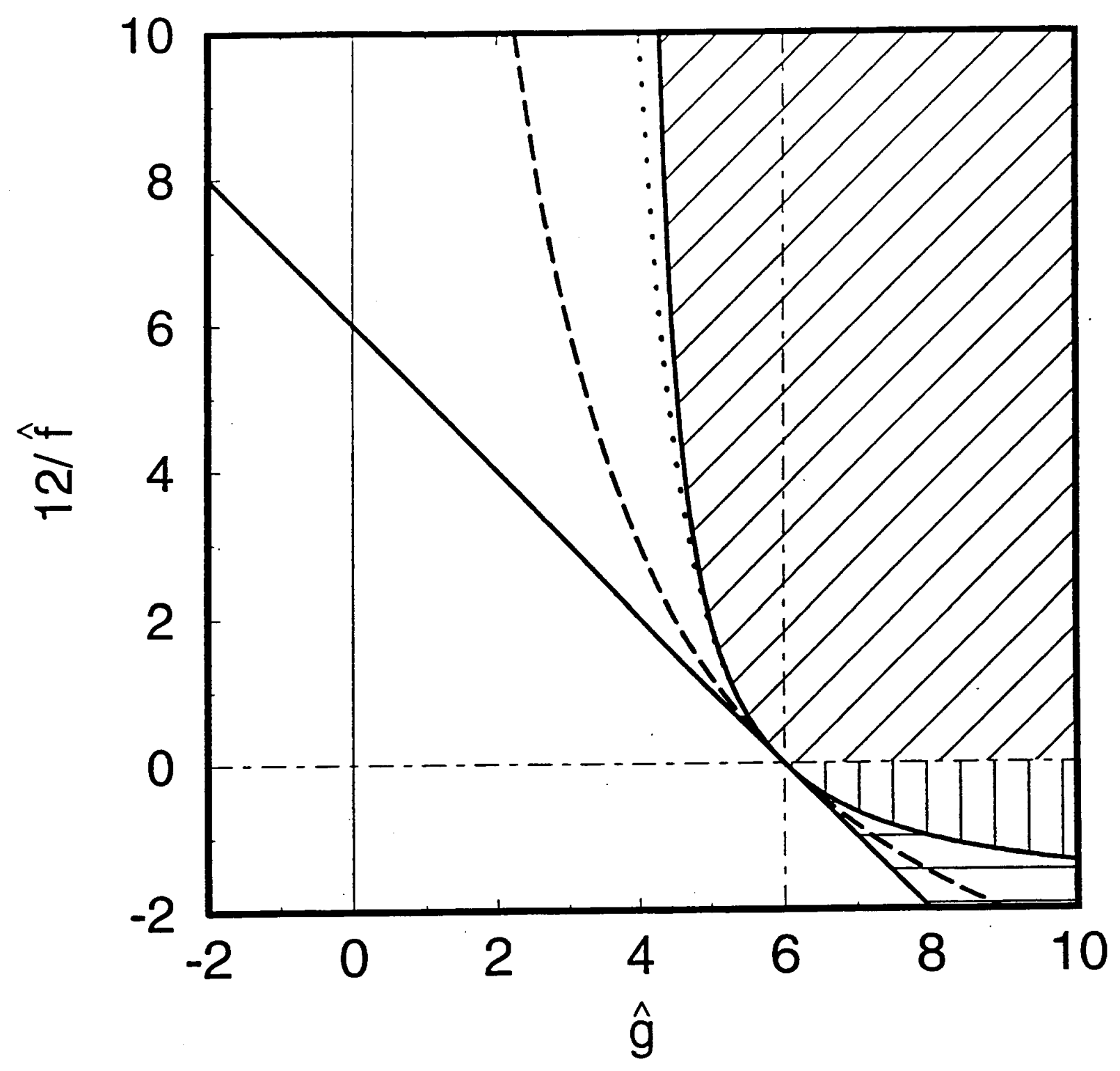

Figure 1. 


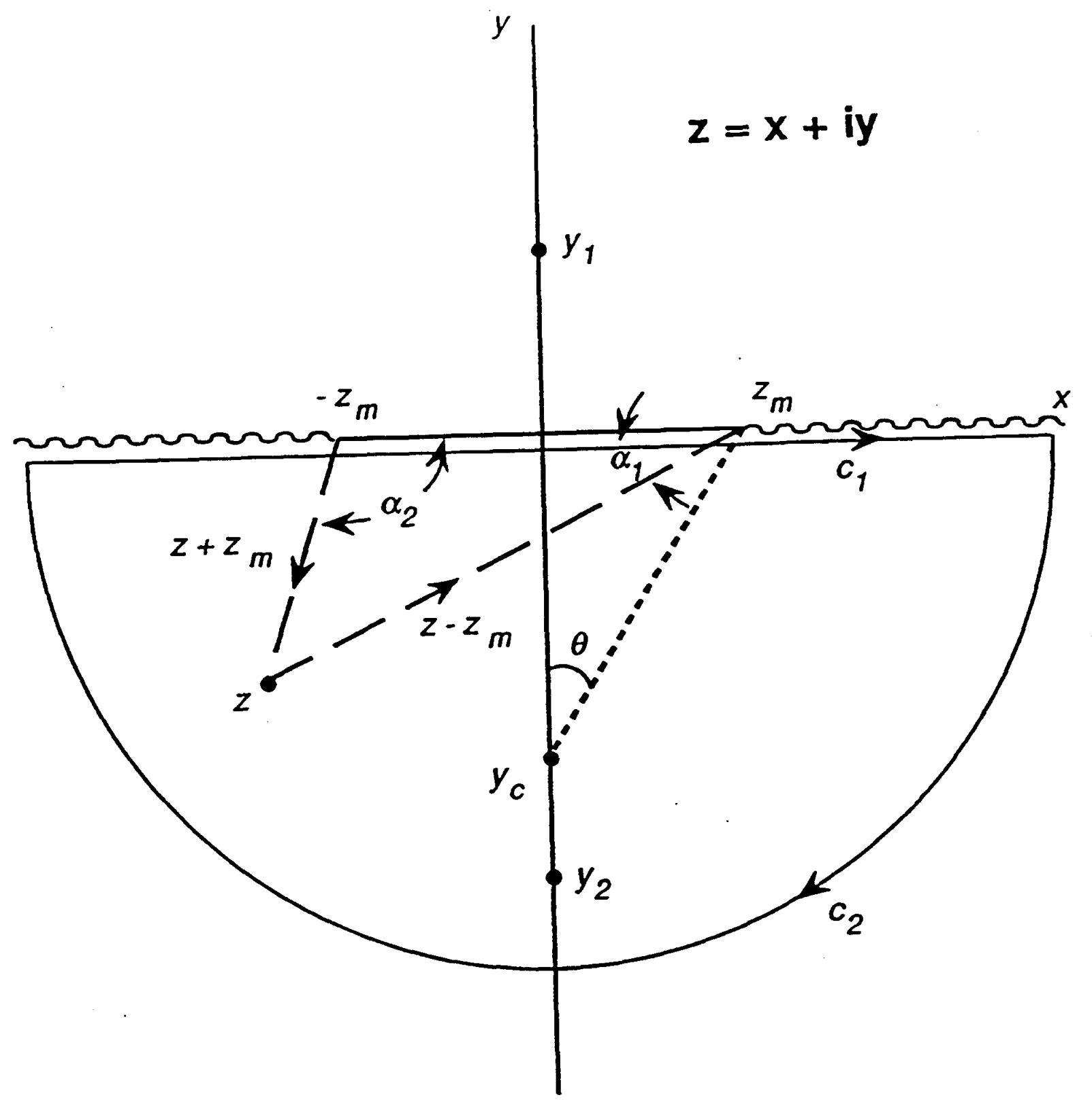

Figure 2. 
(a)

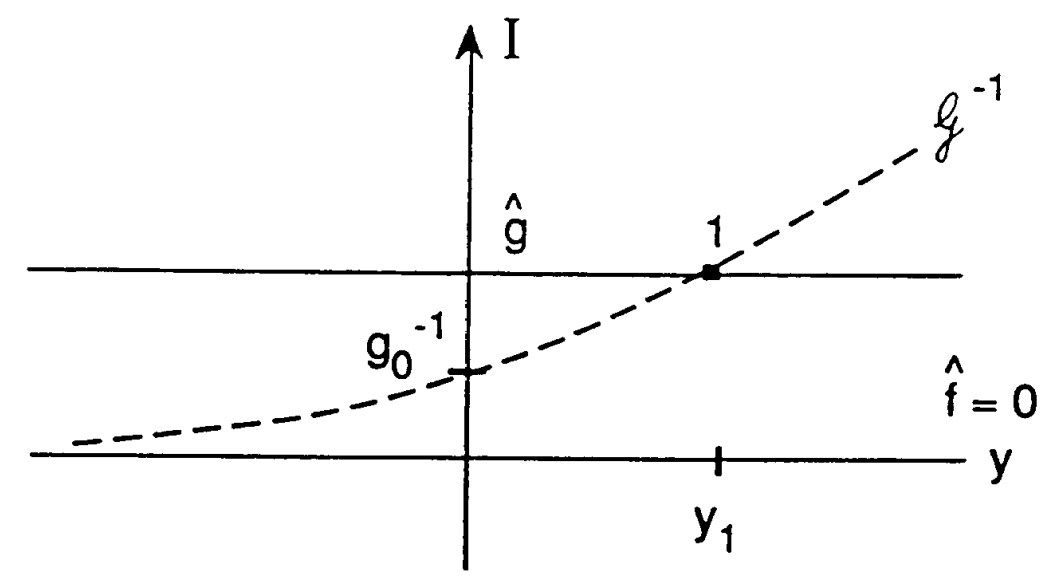

(b)

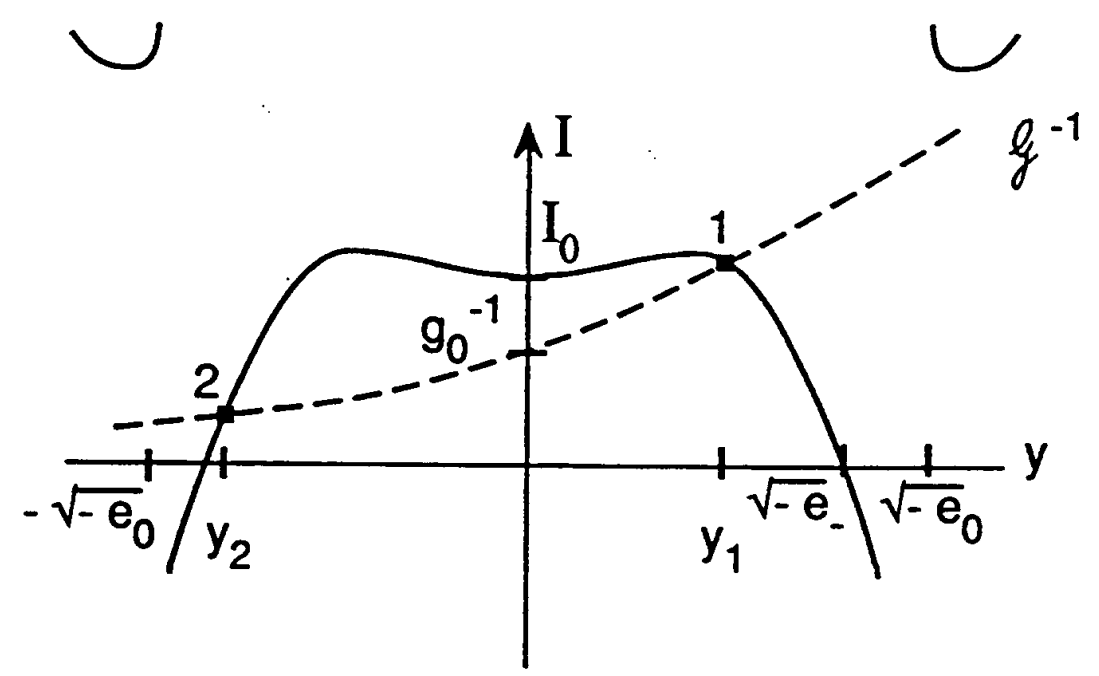

(c)

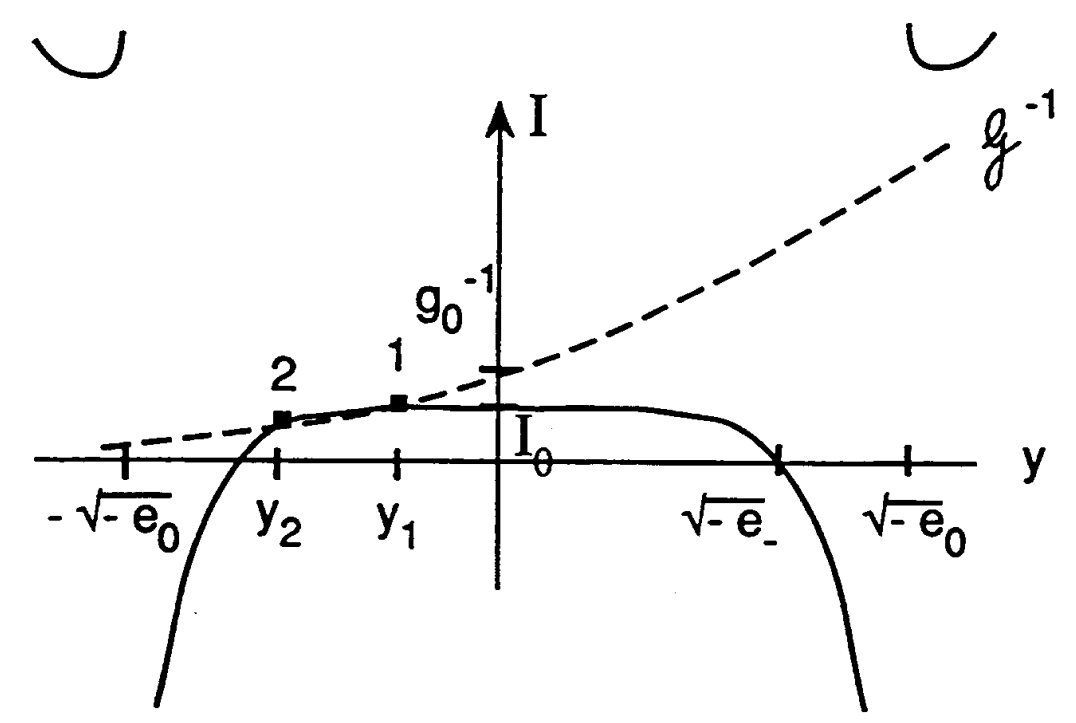

Figure 3. 


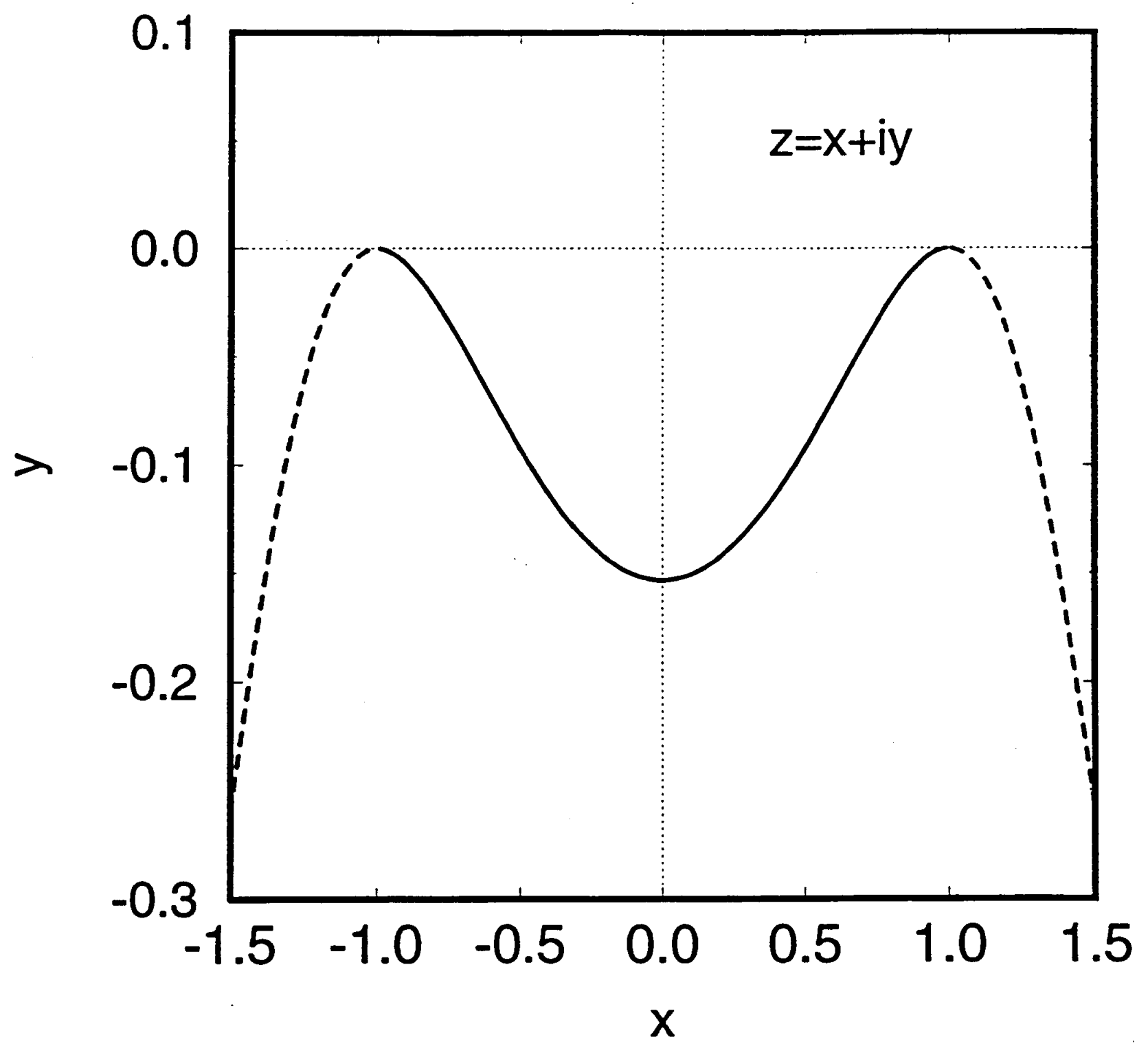

Figure 4. 

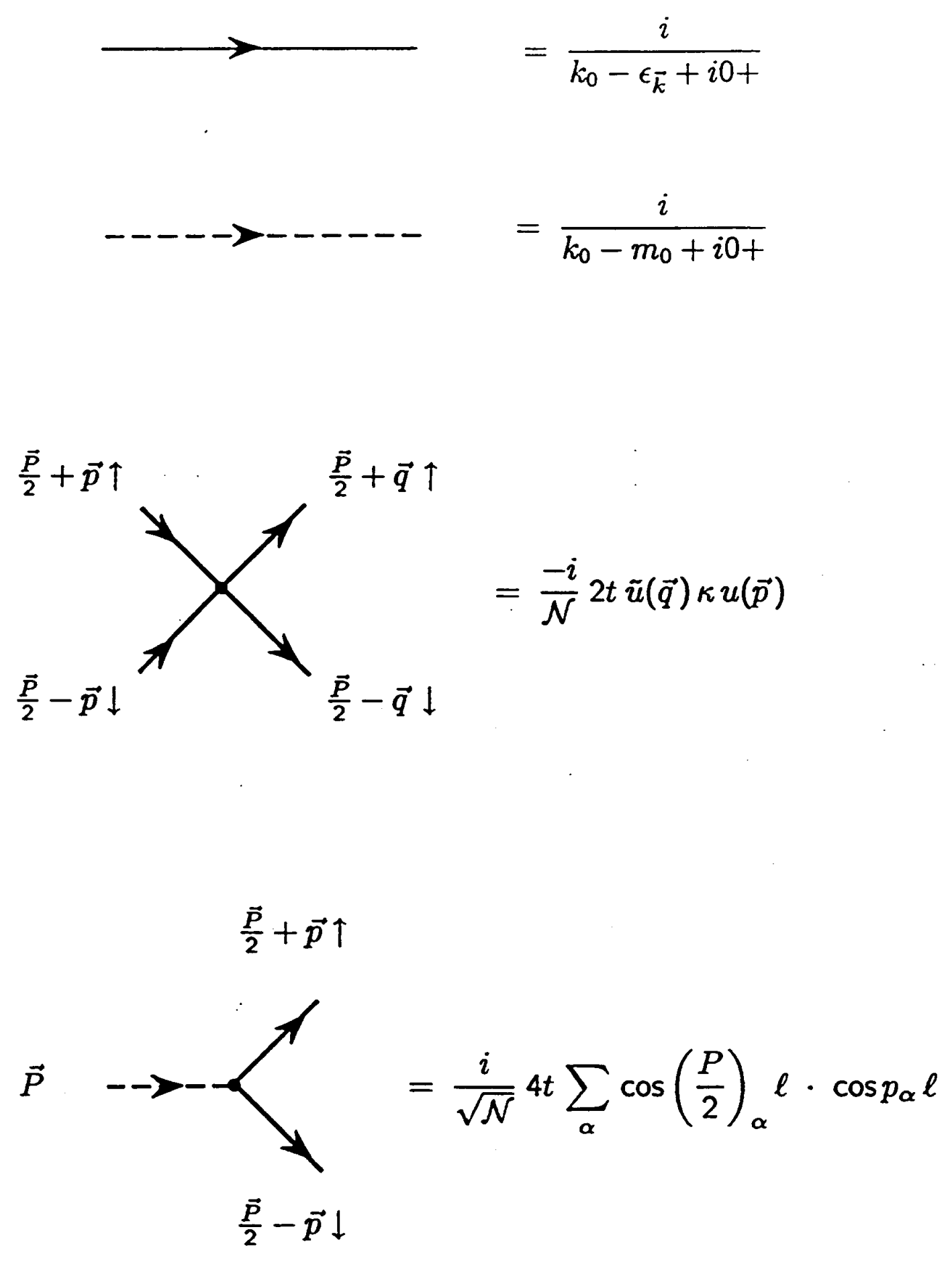

Figure 5. 


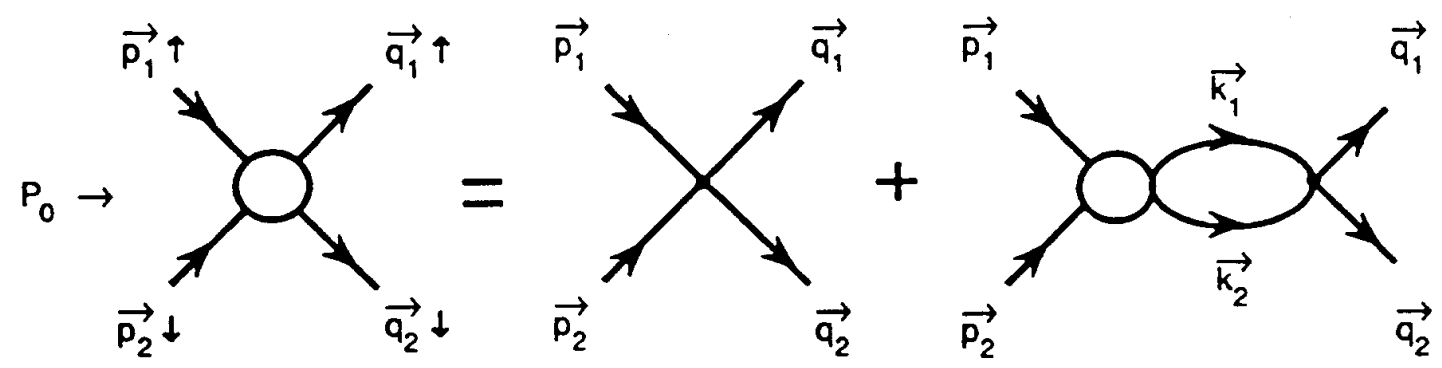

(a)

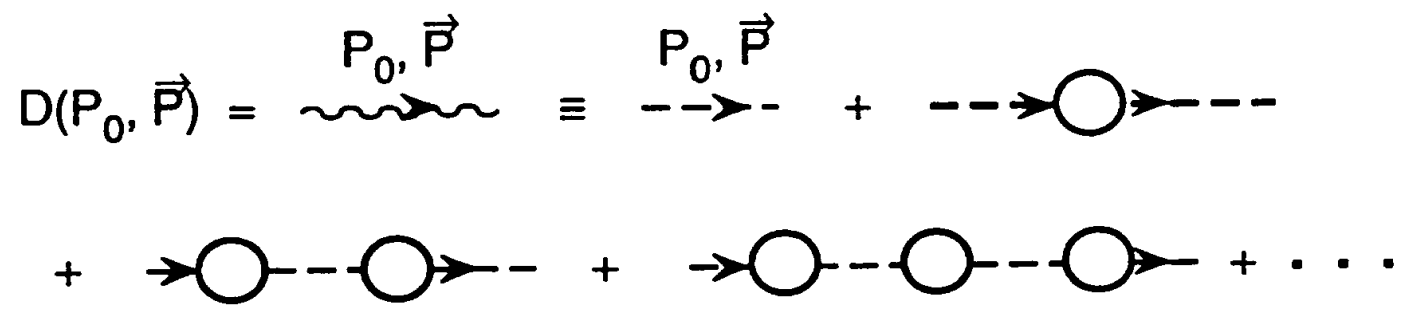

(b)

where

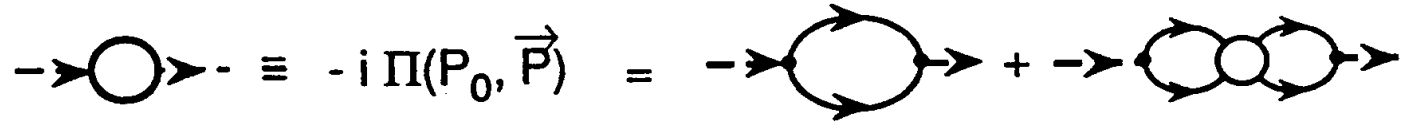

(c)

Figure 6. 

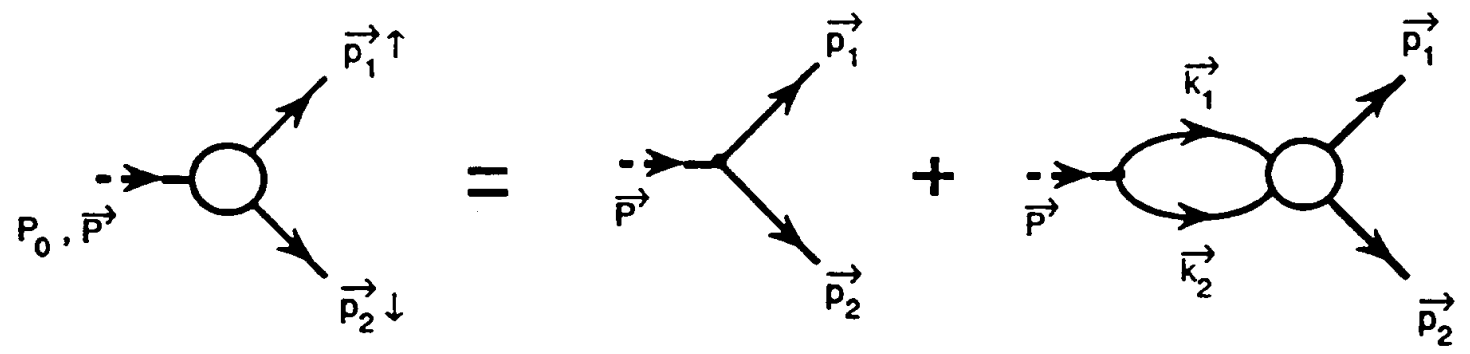

(a)

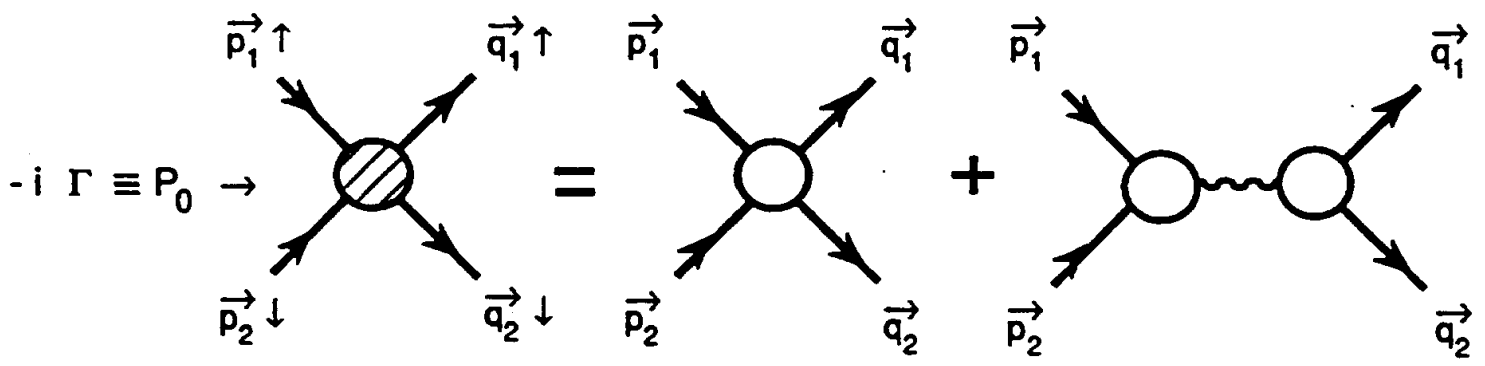

(b)

Figure 7. 


$$
\begin{aligned}
& \frac{\vec{p}}{2}+\vec{p} \quad \frac{\vec{p}}{2}+\vec{q} \\
& =\frac{-i}{r q} \text { 2t } \tilde{U}(\vec{q}) \lambda^{-1} U(\vec{p})
\end{aligned}
$$

$\frac{\vec{p}}{2} \cdot \vec{p}, \sigma \quad \frac{\vec{p}}{2} \cdot \vec{q}, \sigma$

$\frac{\vec{p}}{2}+\vec{p} \quad \frac{\vec{p}}{2}+\vec{q}$

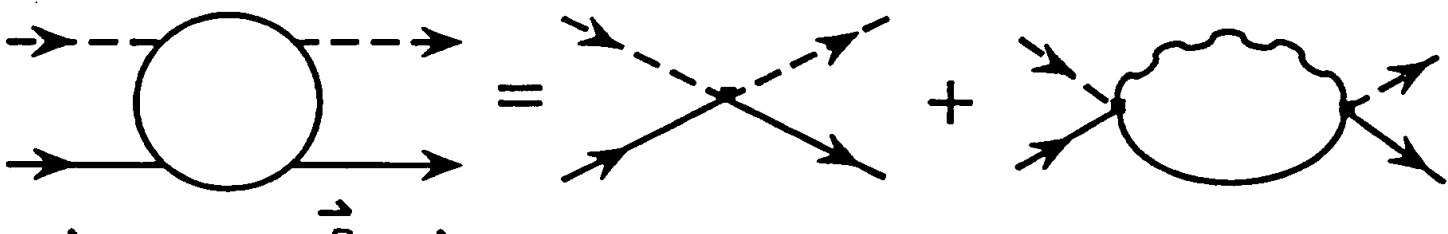

$\frac{\vec{p}}{2} \cdot \vec{p}, \sigma \quad \frac{\vec{p}}{2} \cdot \vec{q}, \sigma$

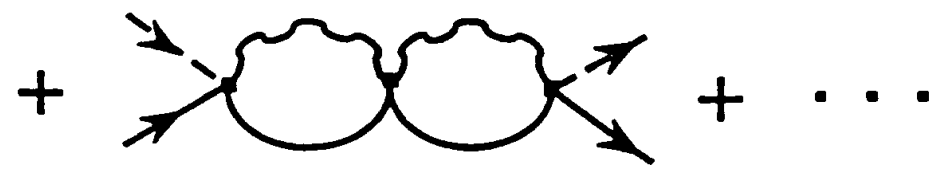

$\frac{\vec{p}}{2}+\vec{p} \quad \frac{\vec{p}}{2}+\vec{q}$
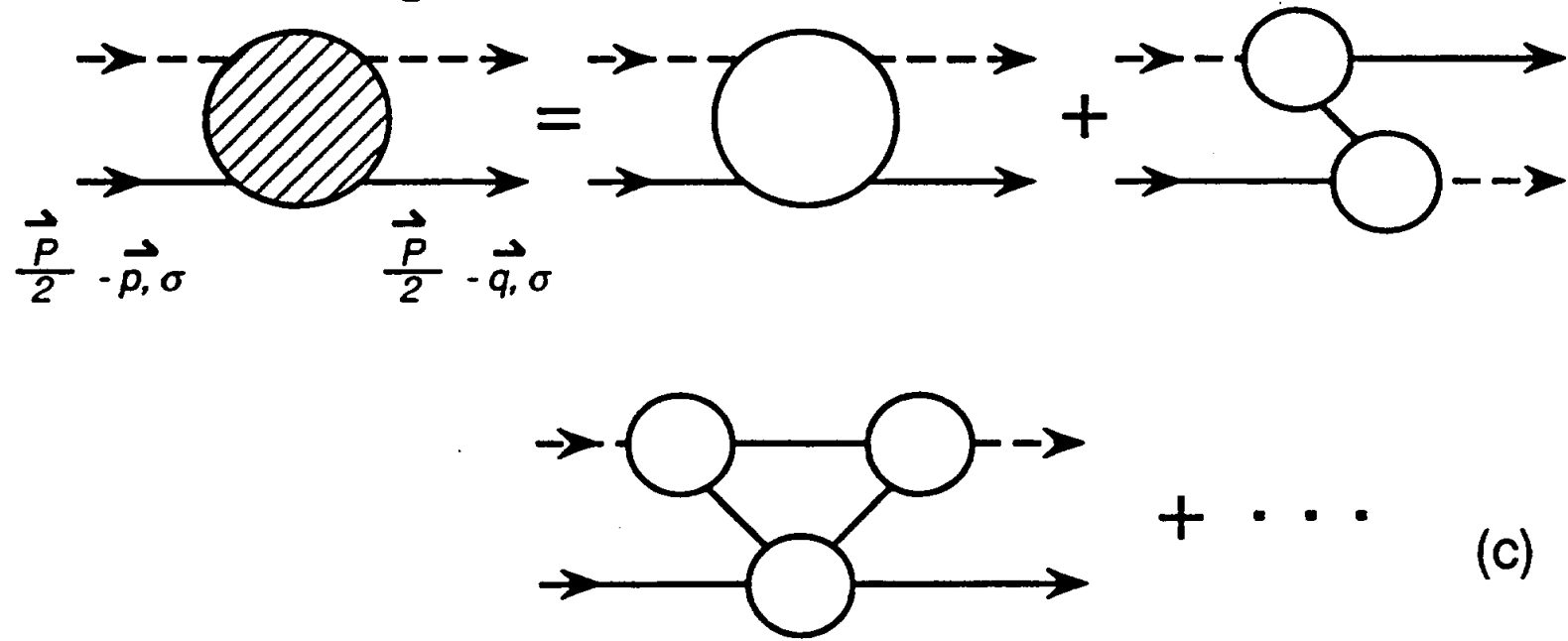

Figure 8. 

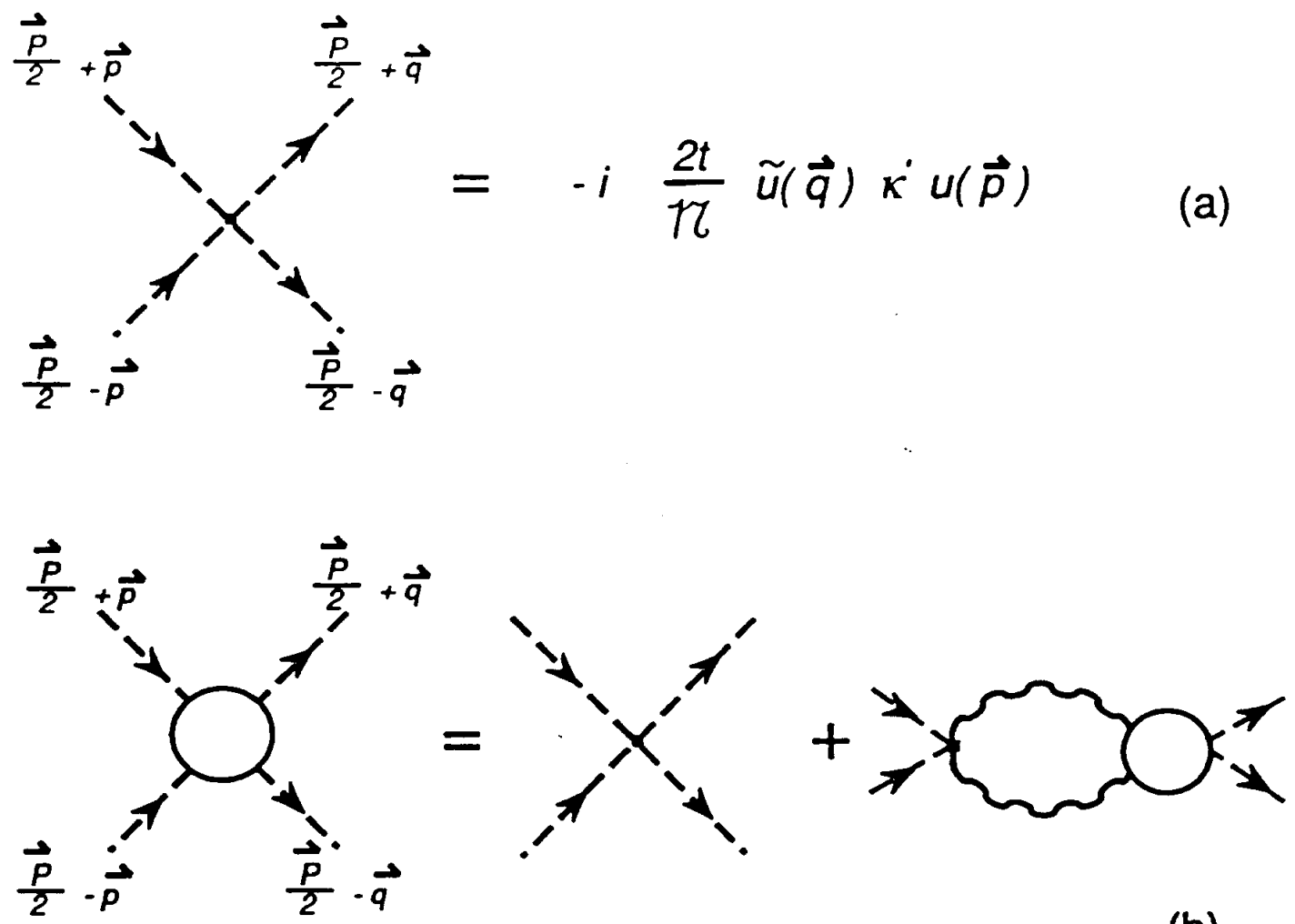

(b)

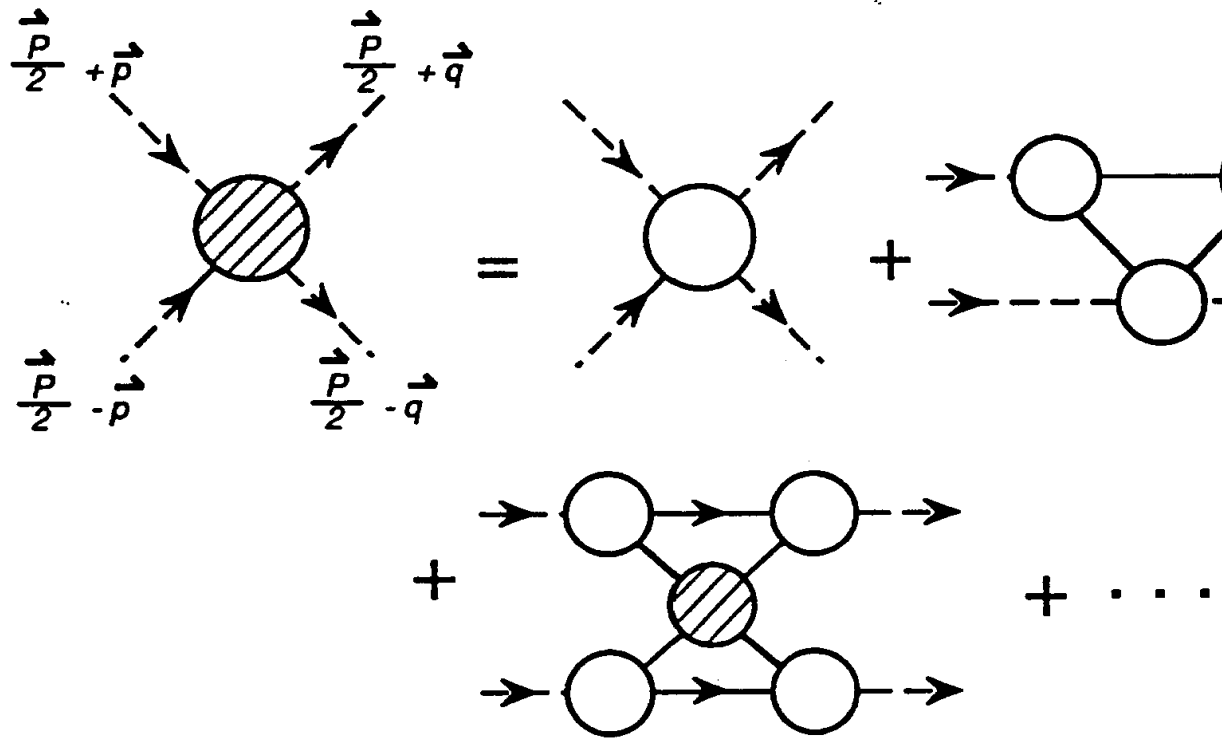

Figure 9. 

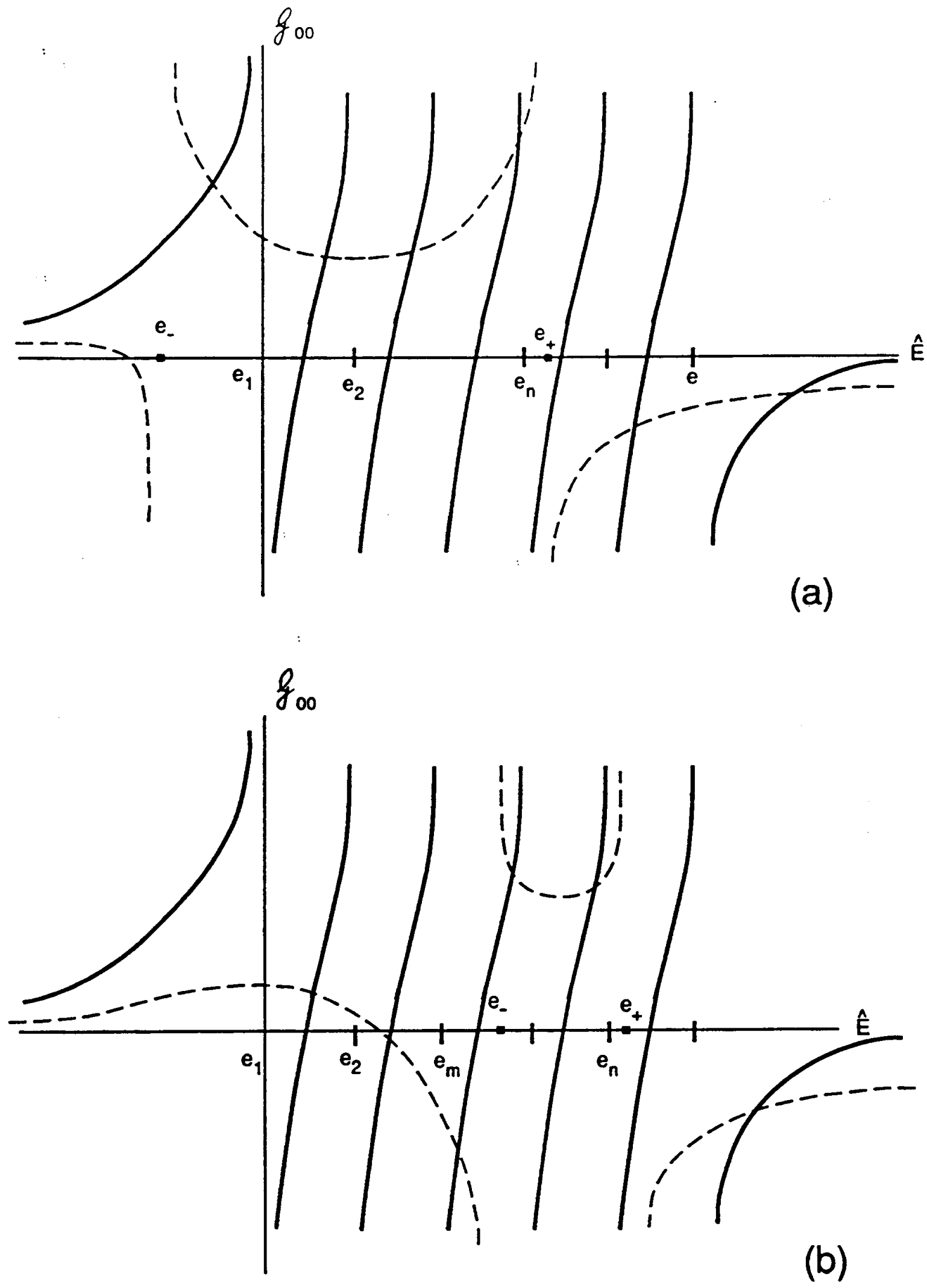

Figure 10. 


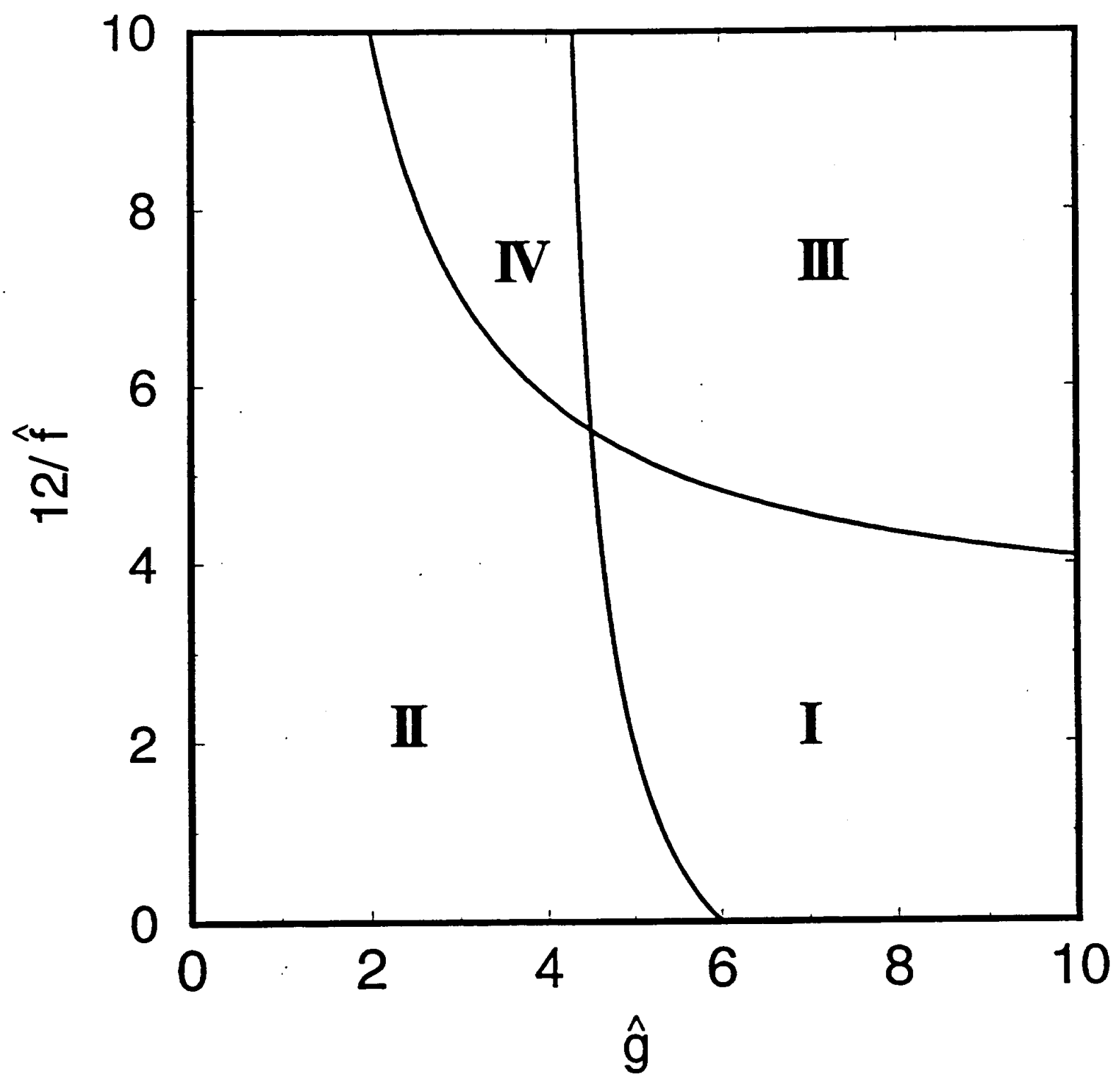

Figure 11. 


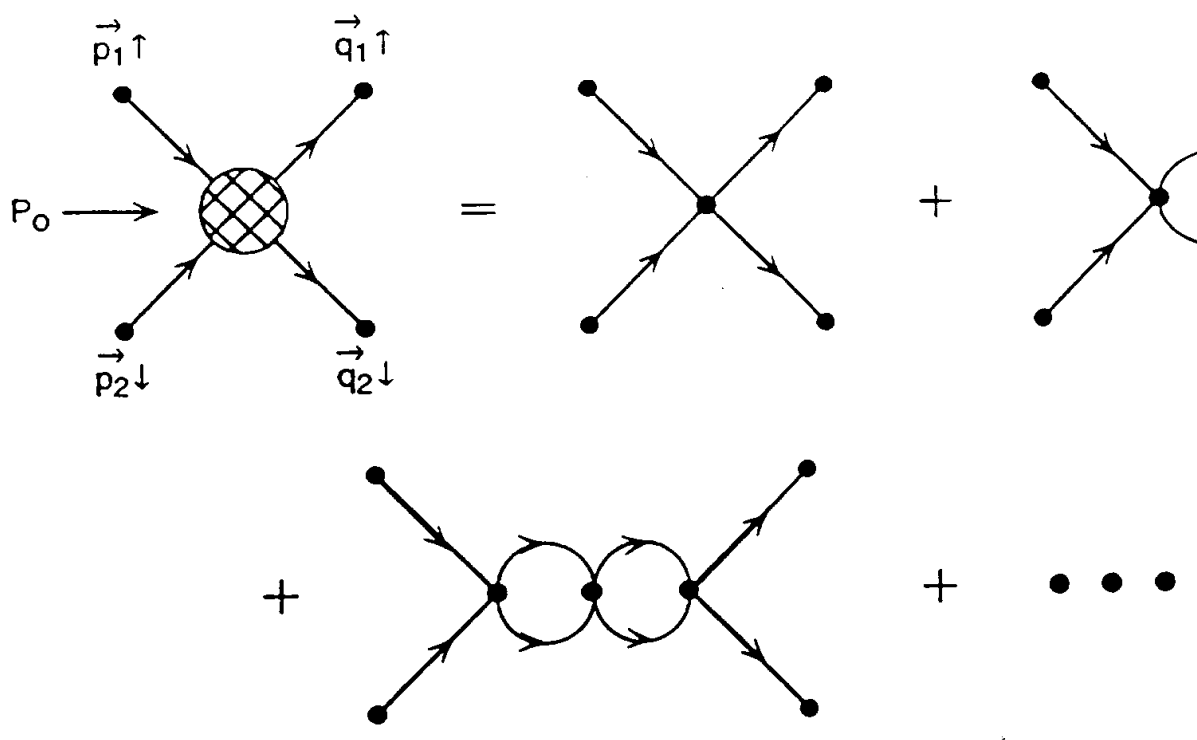

(a)
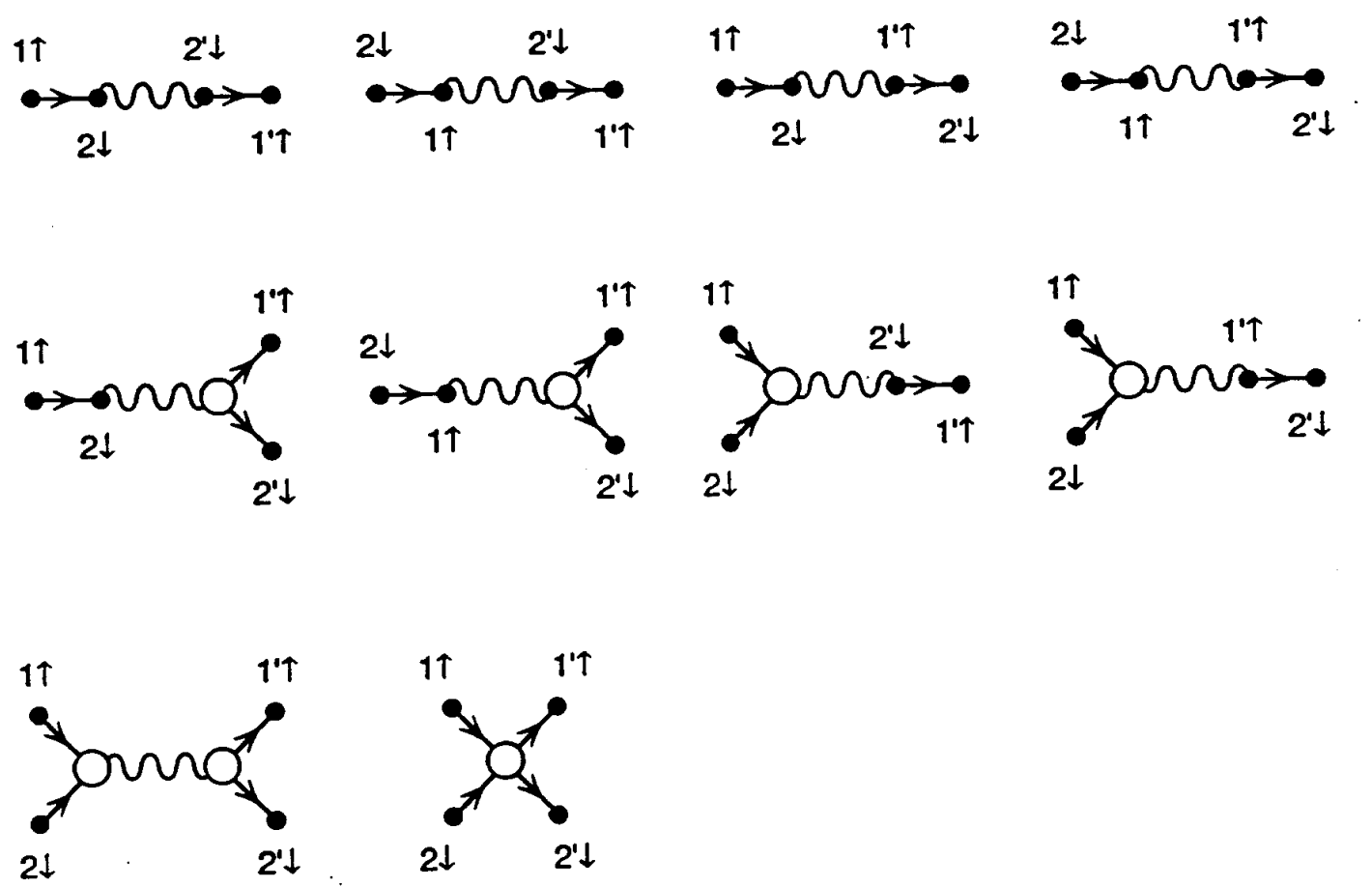

(b)

Figure 12. 